Portland State University

PDXScholar

Spring 6-6-2013

\title{
Lost in the Margins? Intersections Between Disability and Other Non-Dominant Statuses with Regard to Peer Victimization and Psychosocial Distress Among Oregon Teens
}

Marjorie Grace McGee

Portland State University

Follow this and additional works at: https://pdxscholar.library.pdx.edu/open_access_etds

Part of the Disability and Equity in Education Commons, Social Psychology Commons, and the Social Work Commons

Let us know how access to this document benefits you.

\section{Recommended Citation}

McGee, Marjorie Grace, "Lost in the Margins? Intersections Between Disability and Other Non-Dominant Statuses with Regard to Peer Victimization and Psychosocial Distress Among Oregon Teens" (2013). Dissertations and Theses. Paper 1099.

https://doi.org/10.15760/etd.1099

This Dissertation is brought to you for free and open access. It has been accepted for inclusion in Dissertations and Theses by an authorized administrator of PDXScholar. Please contact us if we can make this document more accessible: pdxscholar@pdx.edu. 
Lost in the Margins? Intersections Between Disability and Other Non-Dominant Statuses with Regard to Peer Victimization and Psychosocial Distress Among Oregon Teens

by

Marjorie Grace McGee

A dissertation submitted in partial fulfillment of the requirements for the degree of

Doctor of Philosophy

in

Social Work and Social Research

\author{
Dissertation Committee: \\ Laurie E. Powers, Chair \\ Ben Anderson-Nathe \\ Don Lollar \\ James Nash \\ Matthew Carlson
}

Portland State University

2013 
(C) 2013 Marjorie Grace McGee 


\begin{abstract}
Youth with disabilities experience greater levels of victimization than nondisabled youth. However, little is known about the associations between peer victimization and disability status alone and in combination with sex and race/ethnicity, or with sex and sexual orientation. Further, little is known about the extent to which exposure to peer victimization mediates the relationship between disability status and psychosocial distress. Thus, one purpose of this study was to investigate the extent to which disability status, as a marker of social difference, alone and in combination with other social identities, is associated with differential levels of exposure to peer victimization. A secondary purpose of this research was to examine whether the relationship between disability status and psychological distress is mediated by exposure to peer victimization, and if so, whether the mediation is moderated by sex.

This study analyzed complex survey data, using the 2008 Oregon Healthy Teen dataset, which included 7091 students in $11^{\text {th }}$ grade. Intersectional analyses were conducted to determine the extent to which the student's social status (disability, sex, race, and sexual orientation) was associated with exposure to peer victimization. Results from a series of logistic regressions suggest that disability status is highly associated with exposure to peer victimization. Further, the relationship between disability status and peer victimization changes, and the magnitude of change varies, by specific intersectional status. The relative magnitude of increased odds among students with disabilities reporting peer victimization grew considerably when considered in combination with
\end{abstract}


race/ethnicity and sexual orientation. Results from the mediation analyses confirmed that exposure to peer victimization mediated the relationship between disability status and psychosocial distress; however, there was little support for sex as a moderator.

These findings have the potential to guide development of interventions and strategies (e.g., policies, mechanisms for reporting victimization) to safeguard the health of all students, with particular attention to those at highest risk for peer victimization in the school context. Future research should examine factors in the school environment related to exposure to peer victimization, utilizing an intersectional approach, with attention to differences on multiple non-dominant culture statues. 


\section{DEDICATION}

This dissertation research is dedicated to all students who have experienced peer victimization, especially those most likely to be forgotten or overlooked. I also dedicate this dissertation to those youth with disabilities experiencing adverse circumstances (e.g., victimization, depression, psychosocial distress) who are misunderstood by those around you, and erroneously assumed to be distressed because of your disability. You may "stand out" due to your disability, but your disability does not excuse violence, and psychosocial distress is not inevitable simply because you have a disability.

I also dedicate this research to Rose (my daughter), Dr. Noelle Wiggins (my partner), Dan and Joan McGee (my parents), and Mark and Dr. Michael McGee (my brothers). I am privileged that I came from a family where we were encouraged to go to college, and where we had the means to do so, even if it meant taking out student loans. Therefore, while it was difficult financially for me to pursue a doctorate at mid-career, my family made it possible for me to pursue this dream. I am grateful. 


\section{ACKNOWLEDGEMENTS}

It would not have been possible for me to complete this journey without the support of many people. I am especially grateful for the steadfast support and guidance provided by my advisor and Committee Chair, Dr. Laurie Powers. She was instrumental in sharpening my focus as a scholar and researcher; we had many lively, rich discussions

along the way. She spent many hours reviewing my proposal and dissertation drafts, and helped me draw out the essential "storyline."

I also want to express my appreciation to the dissertation committee. At the beginning of the dissertation proposal process, while Dr. Powers and the rest of the committee (to be named shortly) were supportive, most had reservations about my doing this type of research, due to the complexities inherent in intersectional analyses. I sought to examine the intersections of disability with race and sexual orientation, as well as with sex as a social category. I am appreciative that they supported me in my desire to do this research. If you are not a "quantitative" methodologist, and you find yourself being able to follow the methods and results sections, it is likely due to guidance provided by Dr. Powers and Dr. Jim Nash. In addition to his methodological guidance, Dr. Nash also asked clarifying questions which prompted me to write more clearly so that the reader would understand what I did, and why. If you read, understand, and appreciate the antioppressive threads built into this dissertation, please join me in thanking Dr. Ben Anderson-Nathe, as he challenged me to dig deeper into the messaging surrounding the topic of peer victimization. I am grateful to Dr. Don Lollar for his feedback, which provided a balance between the immediate practicalities of this research, and the 
theorizing. Finally, I appreciate Dr. Matt Carlson, who from the start acknowledged the appropriateness of the research methodology proposed, and later made me aware of the uniqueness of this research.

Of course, no research is possible without data, and in the case of this dissertation, a robust dataset. I am grateful for the Stata support provided by Sarah Ramowski and the information about positive youth development and other adolescent health issues provided by Robert Nystrom, both from the Adolescent Health Program at the Oregon Health Authority (OHA). I am also grateful to other members of the Oregon Preconception Health Action Learning Team, who were instrumental in introducing me to the 2008 Oregon Healthy Teen dataset, which was vital to this dissertation.

My learning curve with Stata (software used for analyses) was made easier due to instrumental support from Dr. Bowen McBeath, faculty at the PSU School of Social Work, and guidance from Dr. Duyen Ngo from Oregon Health Authority. Dr. Kiarri Kershaw, faculty in Preventive Medicine at Northwestern University, was especially helpful in showing me how to test for moderated mediation in Stata. While I did not ultimately conduct multiple imputation, or additional ad-hoc analyses in MPlus, I am grateful to Leif Rustvold and Dr. Paul Swank who spent considerable time sharing their expertise with me. I also have Dr. Amani Nuru-Jeter to thank for the inspiration for a key piece of the theoretical framework; while in Portland for a conference in August of 2010, she provided guidance and suggestions in response to we discussed how I was conceptualizing stressors, such as peer victimization, impact psychosocial distress. 
This journey would have been much more difficult and prolonged without the financial support provided by several entities. I am grateful for the tuition support provided by the Ethel Louise Armstrong Foundation (2006, 2011), and the Robert and Rosemary Lowe Scholarship program (2006). I am also grateful for the tuition benefit granted to employees of the Oregon Health and Science University (2006-2009), and for various graduate (and teaching) assistantships (2009-2012) at the PSU School of Social Work.

I want to acknowledge others who were instrumental at the beginning of my doctoral journey, including Dr. Michelle Berlin, Dr. Lynn Marshall, Dr. Joe Sendelbaugh, and Dr. Jennifer Wisdom. The encouragement from these and other individuals, including my close family and friends, was especially helpful. In particular, there was a point in the application process in which I had grave doubts, and I am grateful for a pivotal conversation I had with Patricia Schoonmaker of the Oregon Health Authority, which convinced me to complete my application to the doctoral program.

Shortly after being accepted into the doctoral program, I co-founded the Women with Disabilities Health Equity Coalition (WowDHEC), with funding from the Johnson \& Johnson Foundation. While I would not necessarily recommend that other doctoral students try to conduct coalition/community building while working on a doctoral degree, I am grateful for support from the many individuals who encouraged my academic pursuits, including WowDHEC staff, Community Health Workers, Board members, as well as staff and faculty from the University of California San Francisco National Center of Excellence in Women's Health. 
While engaged in this dissertation, I found support from members of a strong critical disability studies community which extended far beyond Portland, which included those from the Society for Disability Studies (SDS); these colleagues played a major role in my development as a disability studies researcher and scholar. I am especially grateful for the support and encouragement I received from disability scholars while working on this dissertation from Dr. Beth Omansky, Corbett O’Toole, and Dr. Margaret Price. Another sense of community was provided by Dr. Margrit Eichler and Mary Anne Burke from BIAS FREE Inc (Building an Integrative Analytical System for Recognising and Eliminating inequities). Both were instrumental in sharpening my ability to think about bias in research, and to identify and articulate oppressive bias. Their support went well beyond encouragement; they also made it possible for me to attend two intensive trainings on the BIAS FREE Framework in Canada. The reader may note the application of the BIAS FREE Framework throughout this dissertation, particularly as it shaped my orientation to bias in research. I am also grateful for the encouragement and support from my cohort mates in the doctoral program, as well as other doctoral students from other disciplines, including Rosalyn Taylor, with whom I was able to brainstorm about certain issues in my research process as needed.

I was gifted with a number of writing/research retreats. Specifically I thank Dr. Jutta Ataie for the time spent in the "cabin in the woods," which included long walks and evenings discussing our research dilemmas of the day, and supporting each other as we completed the doctoral program together. I am also grateful to a solo weeklong retreat in the countryside provided by Sue Bisgyer, and another provided by Amalia Alarcón de 
Morris and her family. The time alone, in beautiful spaces, to think, write and work was a blessing. Speaking of beautiful retreats, I would be remiss if I did not mention my parent's wonderful tropical retreat in the "countryside" in Florida.

Finishing this dissertation was made easier with the support of colleagues from the PSU Graduate School of Education, Drs. Pat Burk, and Linda Jessell, as well as the staff from All Hands Raised (formerly the Portland Public Schools Foundation), with whom we worked, who allowed me flexibility as I completed my dissertation research.

I end this rather lengthy acknowledgement with appreciation to Dr. Noelle Wiggins, my partner. Noelle and I were both doctoral students during the first two years of our relationship; I was just beginning, and she was in the last two years of her doctoral journey. It is a bonus that our scholarly interests overlap, with my focus on health disparities, particularly as they relate to people with disabilities, and her focus on community capacity building. We had many meal-time conversations and walks discussing dense academic concepts, and many hours of parallel play related to our academic work. We still do. I also want to thank Noelle for her support which made it possible for me to continue my doctoral studies after my employment ended at OHSU in 2009. She has been quite the cheerleader as well during the past six years. I am grateful for her smile that never fails to make me smile. 
TABLE OF CONTENTS

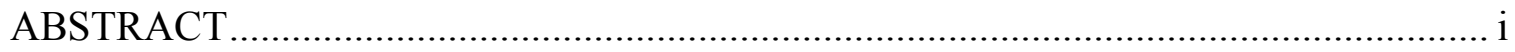

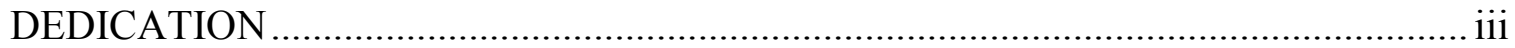

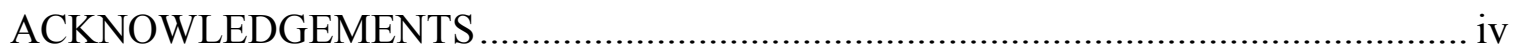

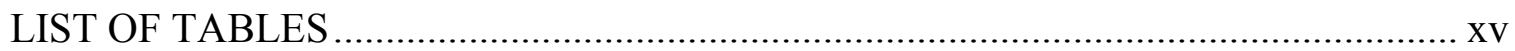

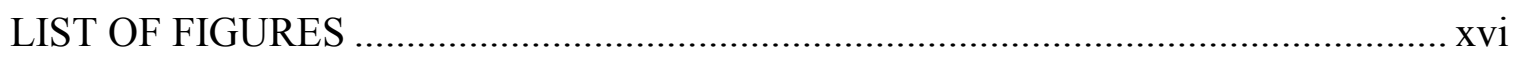

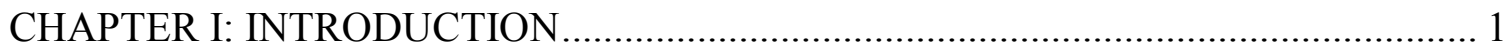

Relationship of Peer Victimization to Psychosocial Distress .............................................. 4

Peer Victimization: Disability and Intersectionality ..................................................... 6

Goals and Objectives for Dissertation Research....................................................... 8

Implications for Practice, Policy, and Research in Social Work and Public Health .......... 9

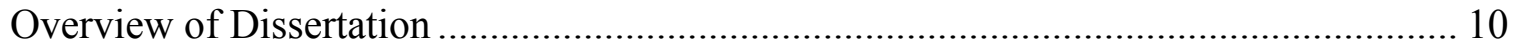

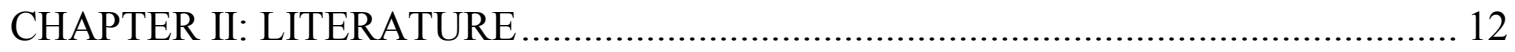

Key Concepts and Inclusion Criteria …………………............................................ 13

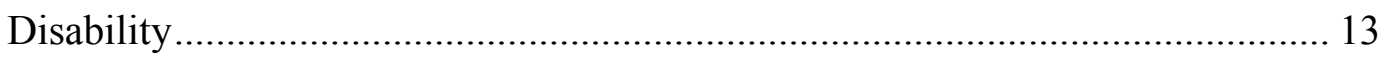

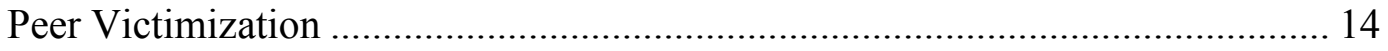

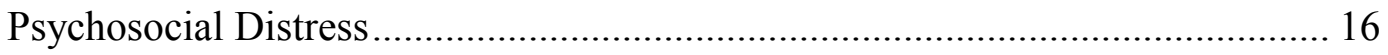

Intersections and Intersectionality ................................................................. 17

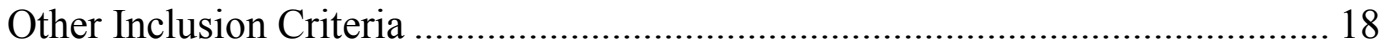

Section I: Associations and Intersections of Disability, Sex, Sexual Orientation, and Race/Ethnicity with Peer Victimization....................................................................... 18

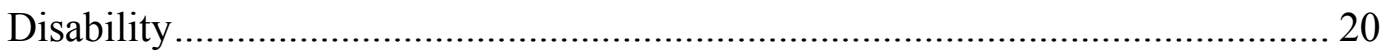

Sex 


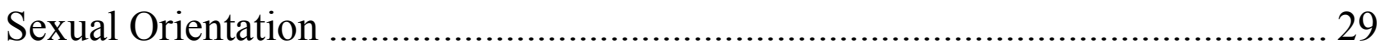

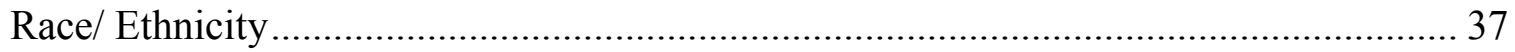

Intersections of Disability and Sexual Orientation .......................................... 42

Intersections of Disability and Race/ethnicity ............................................. 44

Section I Summary: Associations and Intersections .................................... 46

Section II: Psychosocial Distress and Peer Victimization .......................................... 47

Psychosocial Distress: Youth with Disabilities .............................................. 47

Psychosocial Distress: Intersections of Disability and Sex ........................... 52

Peer Victimization and Psychosocial Distress: Youth with Disabilities .............. 55

Peer Victimization and Psychosocial Distress: Mediation and Moderation ......... 61

Section II Summary: Peer Victimization and Psychosocial Distress .................. 64

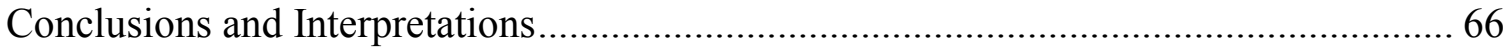

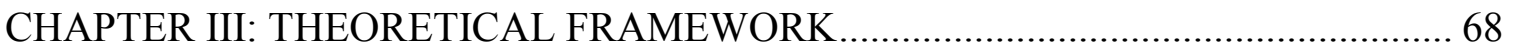

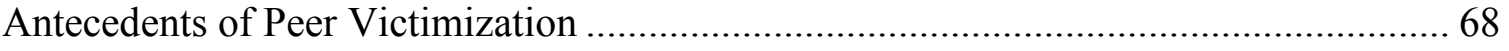

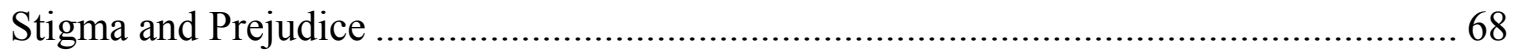

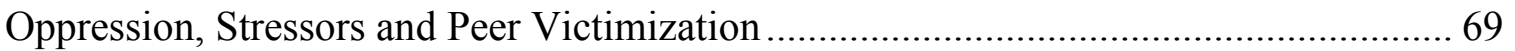

Non-Dominant Culture Status, Social Location, and Intersectionality........................... 73

Goodness-of-Fit with Social Environment, and Exposure to Peer Victimization ............ 75

Connecting Peer Victimization to Psychosocial Distress .......................................... 78 


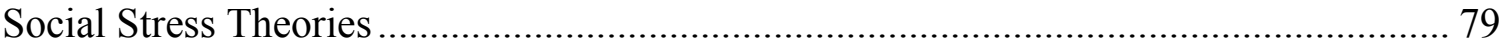

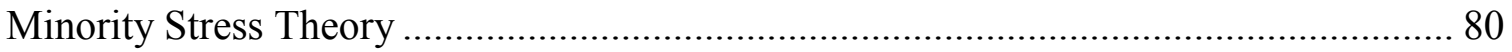

Environment, non-dominant culture status, and identity as antecedents.............. 81

Minority stress processes in response to stressors. ............................................... 81

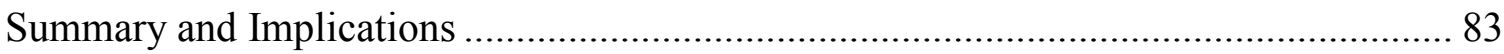

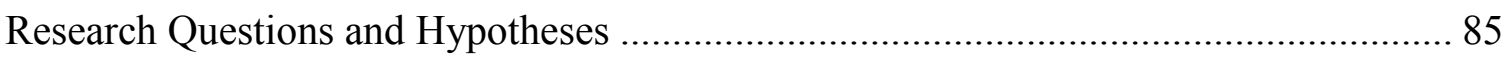

Research Questions and Hypotheses: Aim \#1 ………............................................. 86

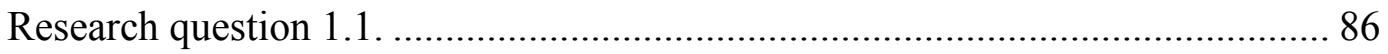

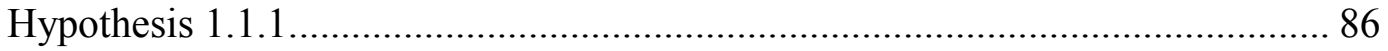

Research Questions and Hypotheses: Aim \#2 ……….............................................. 86

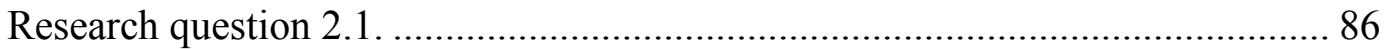

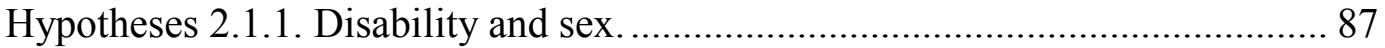

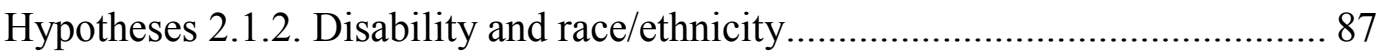

Hypotheses 2.1.3. Disability and sexual orientation......................................... 87

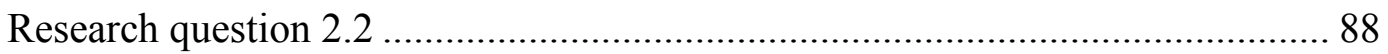

Hypotheses 2.2.1. Disability, sex, race/ethnicity and peer victimization............. 88

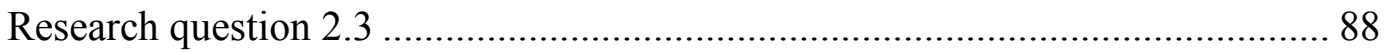

Hypotheses 2.3.1. Disability, sex, sex orientation and peer victimization. .......... 88

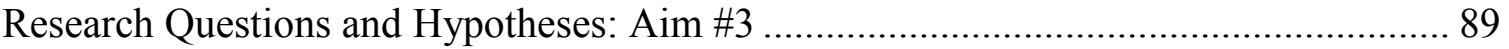




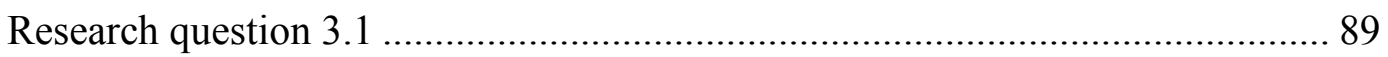

Hypotheses 3.1.1. Disability and psychosocial distress....................................... 89

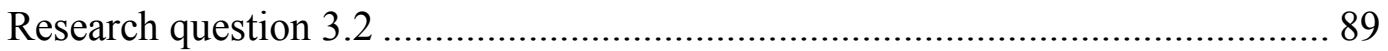

Hypotheses 3.2.1. Mediation: disability, psychosocial distress, and peer victimization

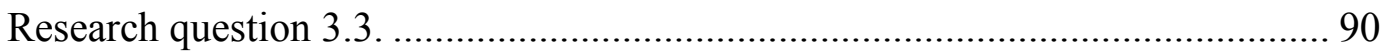

Hypotheses 3.3.1. Moderated mediation: sex, psychosocial distress, and peer

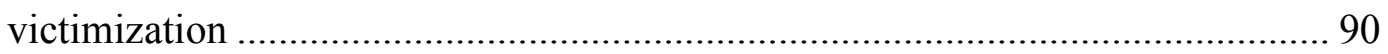

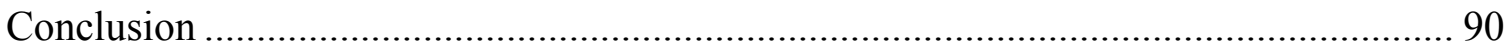

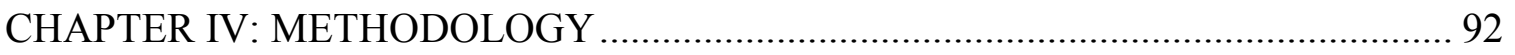

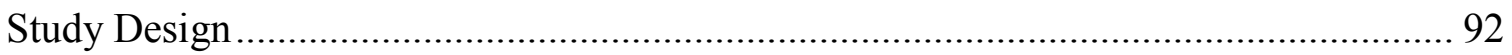

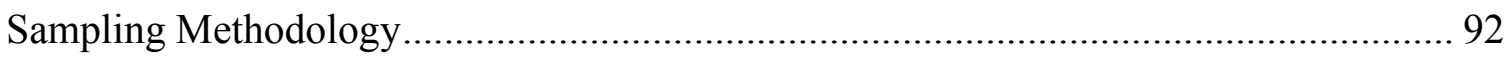

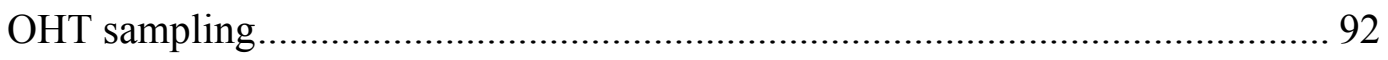

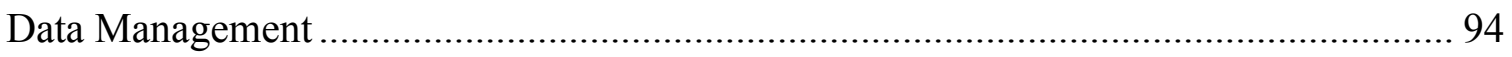

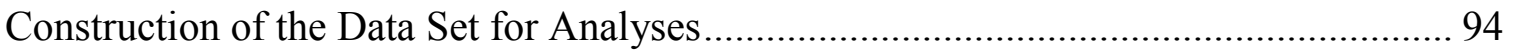

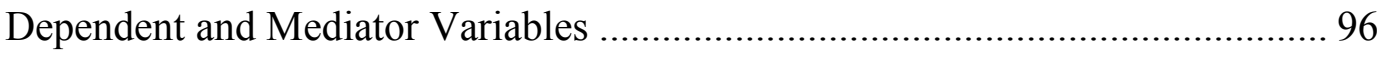

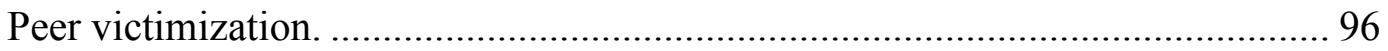

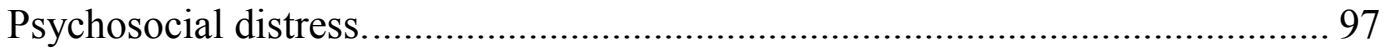

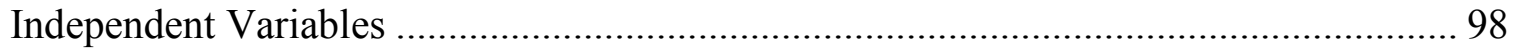

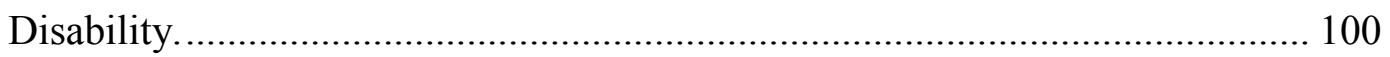


LOST IN THE MARGINS X xiii

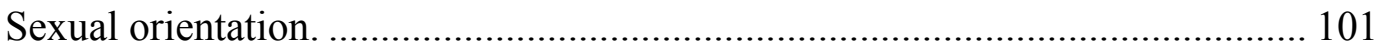

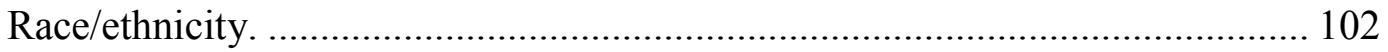

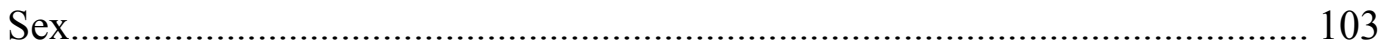

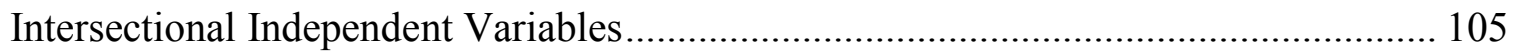

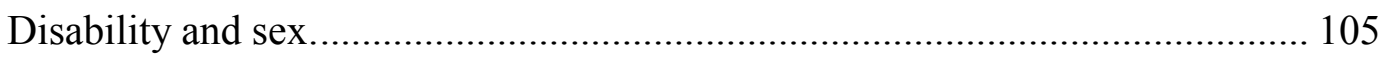

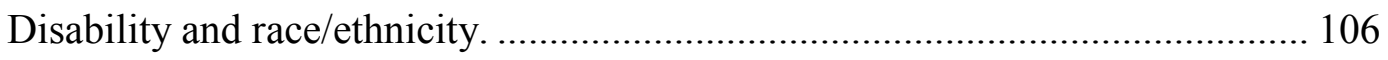

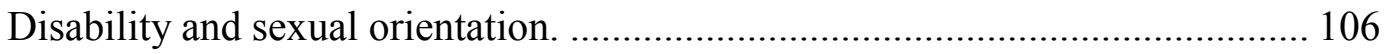

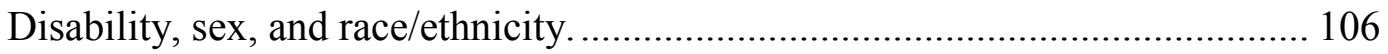

Disability, sex, and sexual orientation. ........................................................ 106

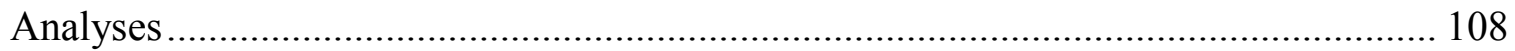

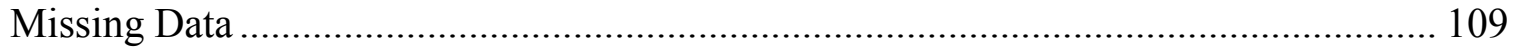

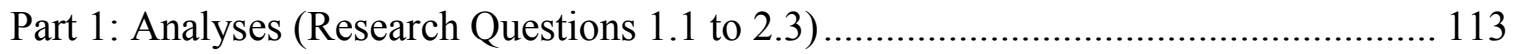

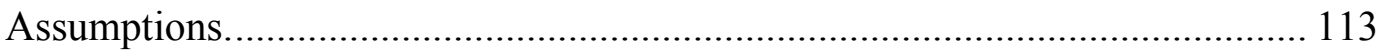

Univariate and bivariate statistics. .......................................................... 114

Multivariate statistics. ........................................................................... 114

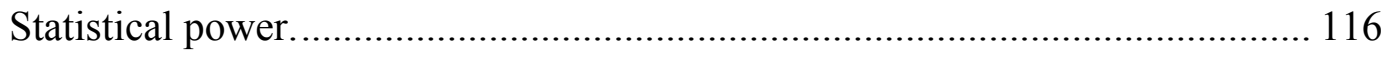

Part 2: Analyses (Research Question 3.1 to 3.3 ) ........................................................ 120

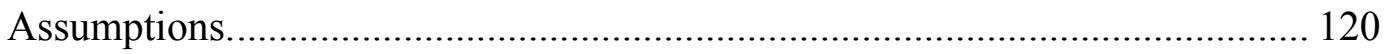

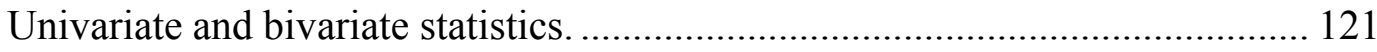


LOST IN THE MARGINS X Xiv

Multivariate analyses. ....................................................................... 121

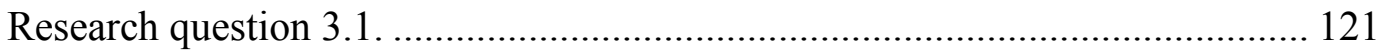

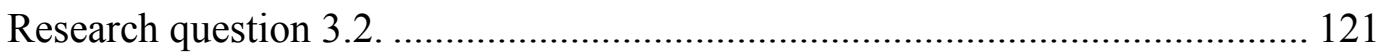

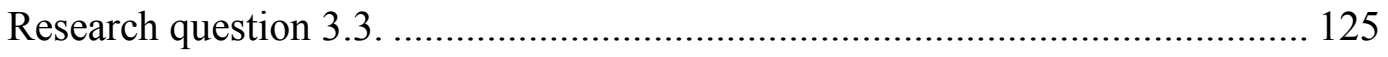

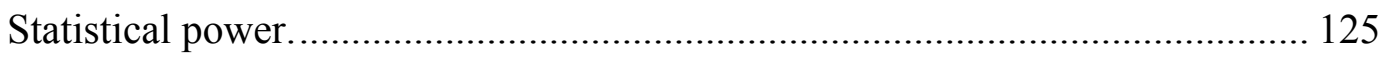

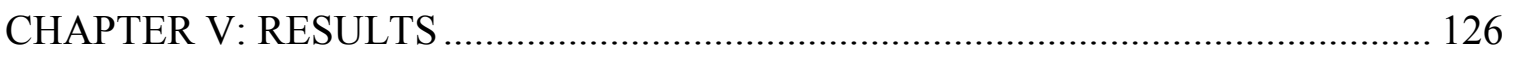

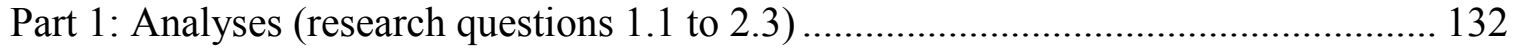

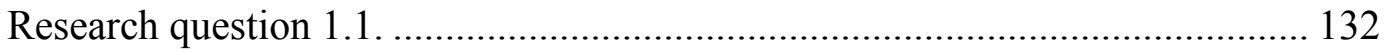

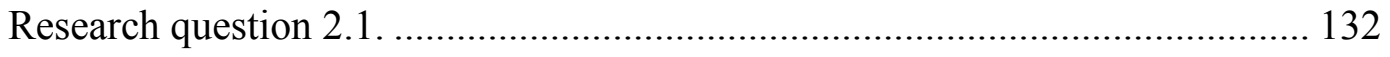

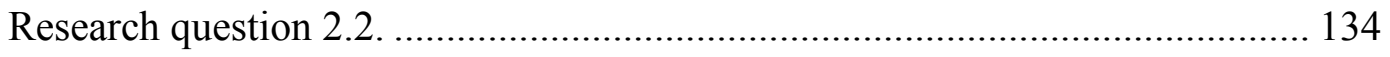

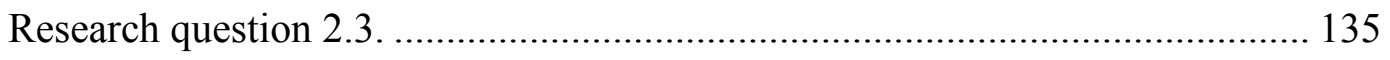

Part 2: Analyses (Research Question 3.1 to 3.3 ) .................................................... 143

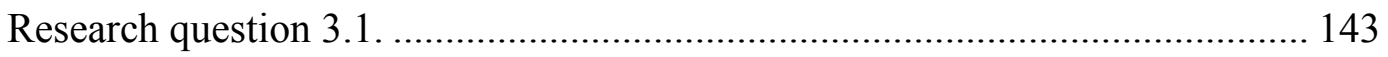

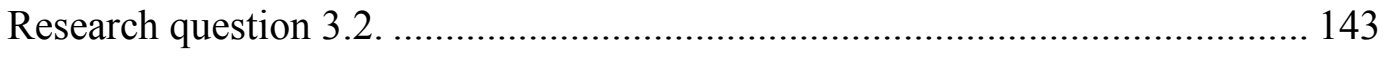

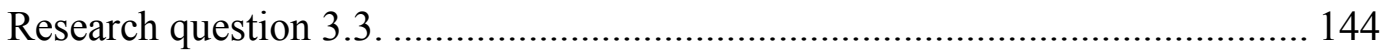

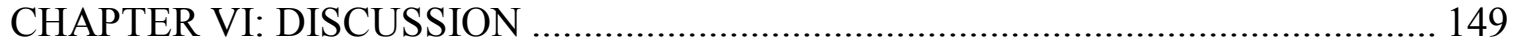

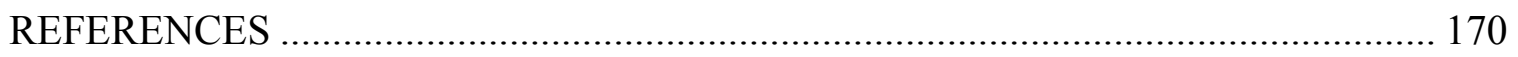

APPENDIX A: Human Subjects Approval from the PSU HSRRC ….......................... 187

APPENDIX B: 2010 Data agreement: Oregon Health Authority ............................... 188

APPENDIX C: 2012 Data agreement: Oregon Health Authority ............................... 189 


\section{LIST OF TABLES}

Table1: Types of Peer Victimization .................................................................... 15

Table 2: Prevalence of Peer Victimization Reported by Disability Status .................25

Table 3: Prevalence of Peer Victimization Reported by Sexual Orientation ..............35

Table 4: Percentage of LGBT Students Reporting Type of Peer Victimization by Sexual Orientation and Sex/Gender Identity...........................................36

Table 5: Prevalence of Peer Victimization Reported by Race/Ethnicity.....................40

Table 6: Percentages of Peer Victimization Reported by Race, and Sex ...................41

Table 7: Percentage of LGBT Students Reporting Peer Victimization Due to Personal Characteristics .....................................................................43

Table 8: Functions of Stigma and Prejudice in Context of Youth with Disabilities

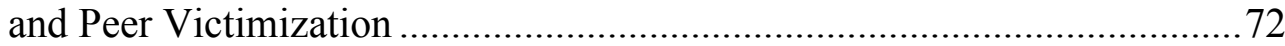

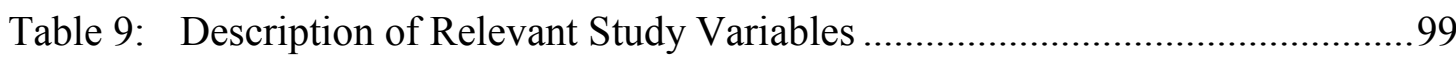

Table 10: Counts \& Weighted Percentages by Selected Key Study Variables .......... 104

Table 11: Counts \& Weighted Percentages of Intersectional Variables ..................... 107

Table 12: Weighted Percentages and Counts of Missingness among Key Variables 111

Table 13: Estimates Used for Effect Size Calculations .......................................... 118

Table 14: Detectable Effect Size Calculations for Logistic Regressions .....................119

Table 15: Characteristics of 11th Graders in Oregon (2008) Reporting Peer Victimization

Table 16: Psychosocial Distress of 11th Graders Reporting Peer Victimization by Sex and Disability

Table 17: Summary of Findings in Context of Aims $1 \& 2$ and Associated Research Questions and Hypotheses...................................................................138

Table 18: Adjusted Odds of Reporting Peer Victimization.......................................139

Table 19: Significance Tests of Exposure to Peer Victimization by Disability, Sex, and Race/Ethnicity

Table 20: Significance Tests of Exposure to Peer Victimization by Disability, Sex, and Sexual Orientation

Table 21: Summary of Findings in Context of Aim 3 and Associated Research Questions and Hypotheses. 145

Table 22: Regression Results from Mediation Analyses

Table 23: Estimated Proportion Mediated and Total Effects 148 


\section{LIST OF FIGURES}

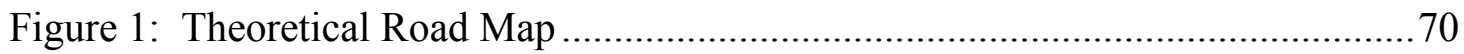

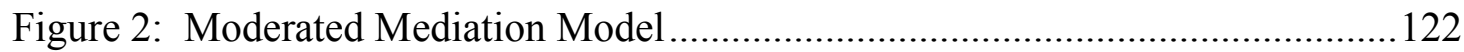

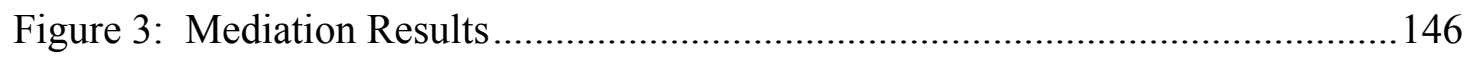




\section{CHAPTER I: INTRODUCTION}

The problems of depression, anxiety, and other types of emotional distress affect many high school students with disabilities. Prevalence estimates by disability status vary considerably, often due to measures designed with different purposes or conceptions of disability (Altman, 2001; Hollar, 2005). Consistent with the International Classification of Functioning, Disability and Health Framework (ICF) published by the World Health Organization (2001), disability is often understood as resulting from a bodily-based impairment; the impairment (e.g., nerve damage in the cochlear) may result in activity limitations, depending on environmental factors such as accessibility (e.g., provision of skilled sign language interpreters) and personal factors (e.g., fluency in sign language). However, the fact that environmental and personal factors can influence one's ability to participate in activities means that an impairment or condition does not always create an activity limitation; thus, perceptions differ about what constitutes a disability and/or an activity limitation. These differing perceptions may account for the wide range of prevalence estimates of disability status among high school students in the U.S. Using a definition that included students having "any physical disabilities or long-term health problems," Jones and Lollar (2008, p. 254) identified a prevalence of 10.3\%. In contrast, Havercamp, Roth, Scandlin, Herrick and Gizlice (2004), found a prevalence of 25\% when they used a definition that included students who said they had at least one of three conditions (activity limitation; difficulty learning, remembering or concentrating due to an impairment or health problem; and/or physical, mental, emotional, or communicationrelated disability). 
For the purposes of this paper, YwD refers to youth who have an impairment (disability) resulting in activity limitations. Youth with conditions such as AttentionDeficit Hyperactivity Disorder (ADHD), cognitive disabilities, and other forms of learning disabilities are included as these conditions make it more difficult to participate fully (activity limitation) in many high school environments.

During the past 20 years, researchers have devoted considerable attention to studying the associations between disability status and psychosocial distress among youth. In this dissertation, the terms "psychosocial distress" and "psychological distress" are used to indicate individual-level negative affective states such as depression, anxiety, and suicidality. Youth with disabilities are significantly more likely than youth without disabilities to report depression (Gortmaker, Walker, Weitzman, \& Sobol, 1990; Havercamp, Roth, Scandlin, Herrick, \& Gizlice, 2004; Jones \& Lollar, 2008; Koenes \& Karshmer, 2000), emotional distress (Blum, Kelly, \& Ireland, 2001; Wolman, Resnick, Harris, \& Blum, 1994), and feeling lonely (Havercamp et al., 2004). There also are sex ${ }^{1}$ differences, with males with disabilities reporting better emotional health than females with disabilities (Huurre \& Aro, 2000; Miauton, Narring, \& Michaud, 2003; Surís, Parera, \& Puig, 1996; Wolman et al., 1994).

Recommendations from this body of research often include the need for linkages to mental health services (Surís, Parera, et al., 1996). When the social context is mentioned in research studies about young people, psychological distress among youth

\footnotetext{
1 I have chosen to use the term "sex" instead of "gender" here to denote biological sex as opposed to gender identity.
} 
with disabilities is often associated with family level factors (Miauton et al., 2003;

Wolman et al., 1994). Recommendations emerging from this research often focus on actions such as improving the youth's ability to interact with peers, provision of supports to parents (Huurre \& Aro, 2000; Stevens et al., 1996), and counseling and guidance to help youth with disabilities and their families (Witt, Riley, \& Coiro, 2003). Such services are important; however, it is notable that little mention is made of the need to explore the relationship between stressors in the environment (such as exposure to peer victimization) and psychosocial distress. Peer victimization refers to behaviors with three elements: repeatedly happening over time, intentionality of harm by bully, and a difference in strength between the "bully" and "victim" (Olweus, 1993). In Chapter Two, I will provide more information about the terms used in this research, such as psychosocial distress and peer victimization.

There is evidence to suggest higher prevalence of peer victimization among $\mathrm{YwD}$, compared to their non-disabled counterparts. Studies based on convenience samples consistently report higher rates of peer victimization among children and teens with diverse disabilities (Dawkins, 1996), including those with ADHD (Unnever \& Cornell, 2003; Wiener \& Mak, 2009), and visual impairments (Horwood, Waylen, Herrick, Williams, \& Wolke, 2005). A 2006 study of over 12,000 students aged 11, 13, and 15 in France and Ireland included 2,026 students with disabilities. In France, significantly higher proportions of male (41\%) and female (41\%) students with disabilities reported peer victimization, compared to non-disabled males (31.8\%) and females (33.9\%) (Sentenac et al., 2011). In Ireland, the overall percentage of students reporting peer 
victimization was higher among female (31.1\%) and male (29.4\%) students with disabilities, compared to non-disabled female students (23.6\%), and non-disabled male students (26.1\%) (Sentenac et al., 2011). Closer to home, a report based upon the 2011 Montana Youth Risk Behavior Survey (YRBS) survey cited 38.3\% of students with disabilities as being harassed compared to $26 \%$ of their nondisabled peers (Montana Office of Public Instruction, 2011). Common to these studies, however, is a lack of focus on peer victimization as a factor influencing psychosocial distress among youth with disabilities.

Despite empirical evidence suggesting increased psychosocial distress as well as increased exposure to peer victimization among YwD compared to their peers, specific mention of peer victimization, either as an antecedent or consequence of increased psychosocial distress among YwD, compared to youth without disabilities, is nearly absent from the literature. This gap in the literature impedes addressing the problem of psychosocial distress among youth with disabilities.

\section{Relationship of Peer Victimization to Psychosocial Distress}

There is a robust literature linking psychosocial distress and peer victimization among non-disabled students. For example, Hawker and Boulton (2000) conducted a comprehensive meta-analysis of 23 studies on the relationship of victimization to psychosocial distress, spanning 20 years from 1978 to 1997, and concluded that sufficient research has been done to document the ill effects of victimization on the psychosocial health of youth. Hawker and Boulton (2000) recommended future research examining more complex questions related to peer victimization, such as risk factors and causation. 
Since 2000, a number of researchers heeded these recommendations. For example, several studies suggests that sex moderates the relationship between exposure to peer victimization and psychosocial distress, with females exposed to peer victimization being more likely to report symptoms of psychosocial distress compared to males exposed to peer victimization (Dao et al., 2006; Nabuzoka, Rønning, \& Handegård, 2009). Researchers also have studied the effects of peer victimization across subgroups, and tested non-dominant sexual identity as a moderator on the relationship between peer victimization and psychosocial distress. For example, the study by Espelage, Aragon, Birkett and Koenig (2008) which found that LGBQ students exposed to high levels of homophobic teasing reported higher levels of psychosocial distress, compared to heterosexual students exposed to high levels of homophobic teasing. Gruber and Fineran (2008) found that females and GLBQ students reporting sexual harassment also reported significantly higher levels of psychosocial distress, compared to males reporting sexual harassment.

In contrast to this robust body of research examining the effects of peer victimization on the psychological health among youth overall, and among youth in certain non-dominant groups, ${ }^{2}$ relatively little research has been conducted characterizing peer victimization as an exposure variable associated with psychosocial distress among YwD. For example, while some researchers explored the prevalence and effects of victimization generally among youth, they have not investigated associations between

${ }^{2}$ As the term "minority" usually refers to a group of people, based upon a social identity or characteristic, that is smaller, the term "minority" can be problematic. Therefore, following the example set by the authors of the BIAS FREE (Building an Integrative Analytical System For Recognizing and Eliminating InEquities) Framework (Burke \& Eichler, 2006), I use the term "non-dominant" to refer to those groups who, relative to a dominant group, are subordinate. 
peer victimization and psychosocial health among YwD (see for example: AlrikssonSchmidt, Armour, \& Thibadeau, 2010; Blum et al., 2001; Reiter, Bryen, \& Shachar, 2007).

Only a few studies specifically examined the association of peer victimization and psychosocial distress among children and teens with disabilities (Baumeister, Storch, \& Geffken, 2008; Humphrey, Storch, \& Geffken, 2007). None of these studies examined the impact of peer victimization on the psychological health of YwD, though one study examined the association for adults with intellectual disabilities (Emerson, 2010), and another focused on adults with speech difficulties (Hugh-Jones \& Smith, 1999). This may be due in part to a lack of existing data sets, and/or to a lack of recognition of YwD as a group experiencing forms of oppression such as peer victimization. For example, in a study that focused on sexual violence among adolescent females with disabilities, the authors reported significant correlations between being forced to have sex, and experiencing bullying, feeling sad, and use of alcohol, tobacco and other drugs (ATOD). However, the authors did not report on the correlations of bullying, feeling sad, and use of ATOD by disability status (Alriksson-Schmidt et al., 2010).

\section{Peer Victimization: Disability and Intersectionality}

Disability, like race, sex, and sexual orientation, is a social construct with meanings that can produce experiences of oppression (Crenshaw, 1991), such as peer victimization. In other words, if members of the dominant group (in this case, people without disabilities) construe disability as negative, then people with disabilities may be more likely to experience discrimination, a manifestation of oppression. Multiple 
stigmatized identities can result in increased exposure to peer victimization. As will be discussed in greater depth in Chapter Two, prevalence of peer victimization is often significantly higher among marginalized groups relative to their non-marginalized peers. Research suggests that the following groups are more likely to report victimization: YwD compared to non-disabled peers (Carroll \& Shute, 2005); lesbian, gay, or bisexual youth compared to youth who identify as heterosexual (Berlan, Corliss, Field, Goodman, \& Austin, 2010; Bontempo \& D'Augelli, 2002; Espelage et al., 2008; Gruber \& Fineran, 2008; Hatzenbuehler, 2011; Young \& Sweeting, 2004) and Latina/o and multi-racial youth compared to white youth (Sawyer, Bradshaw, \& O'Brennan, 2008; Wang, Iannotti, Luk, \& Nansel, 2010).

Further, exposure to peer victimization appears to vary by sex across all identities. Females who are disabled, black, Asian, Latino, or multi-racial appear to be more likely to report peer victimization compared to males who are disabled, black, Asian, Latino, or multi-racial (Sawyer et al., 2008; Sentenac et al., 2011). Gay and bisexual males, on the other hand, are more likely to report peer victimization compared to lesbians and bisexual females (Kosciw, Greytak, Diaz, \& Bartkiewicz, 2010).

However, research gaps persist, particularly in terms of whether disability status in addition to sexual/gender and/or racial/ethnic non-dominant status is associated with increased exposure to peer victimization. For example, we do not know if youth of color with disabilities, or lesbian, gay, and bisexual (LGB) youth with disabilities are at more risk for exposure to peer victimization, compared to youth of either status considered 
alone. Lesbian, gay, bisexual, transgendered, queer, and questioning (LGBTQ $\left.{ }^{3}\right)$ youth with disabilities and youth of color with disabilities are virtually invisible in the current victimization research.

To summarize, a number of researchers have studied the prevalence of peer victimization and its impact on psychosocial health in the general population, as well as in certain subpopulations, such as LGB youth. However, little is known about the intersections of disability and other status indicators in explaining peer victimization. Further, it is notable that while there is much research showing that $\mathrm{YwD}$ experience greater levels of victimization and psychosocial distress, there is relatively little research directly connecting their victimization by peers to psychosocial distress.

\section{Goals and Objectives for Dissertation Research}

The overarching goal of this study was to advance understanding of whether disability status, as a marker of social difference, alone and in combination with other social identities, is associated with differential levels of exposure to peer victimization. Additionally, I examined whether the relationship between disability status and psychological distress was mediated by exposure to peer victimization. The three specific aims of this research were:

1. To examine the extent to which disability status is associated with exposure to peer victimization;

\footnotetext{
${ }^{3}$ The term LGBTQ is often used to refer to both sexual (LGB; lesbian, gay, bisexual) and gender (T; transgender) minorities, as well as "not sure" $(\mathrm{Q}=$ questioning; $\mathrm{Q}$ is also used by some to denote "queer" and may show up along with a $\mathrm{Q}$ for questioning as in "LGBTQQ") students.
} 
2. Taking an intersectional approach, to investigate the extent to which disability status in combination with two sets of identity variables (sexual orientation, sex; and race/ethnicity, sex) is associated with exposure to peer victimization.

3. To determine if disability status is associated with psychosocial distress, and if so, determine if the effects of disability status on psychosocial distress are mediated by exposure to peer victimization.

\section{Implications for Practice, Policy, and Research in Social Work and Public Health}

As will be seen in Chapter Two, far less research has been conducted about prejudice based on disability status than about prejudice based on other marginalized identities. It is time to examine the relationship between exposures to stressors such as peer victimization, and psychosocial distress among youth with disabilities. For example, in the process of addressing the first two aims of this study- to examine the extent to which disability status alone and in combination with sexual orientation and sex, and with race/ethnicity and sex statuses, is associated with exposure to peer victimization - this research examined the increased risk of exposure to peer victimization among those who may have previously been "invisible" (Crenshaw, 1991). This work helps elucidate the varying possible relationships between multiple stigmatized identities and peer victimization. Understanding more specifically who is at increased risk for exposure to peer victimization, is useful for all professionals working to promote health among disabled and sexual non-dominant culture youth. Interventions can be designed to provide levels of protections (e.g., policies, access to counseling, support groups and health promoting information, mechanisms for reporting victimization, and so on) to safeguard 
the social health of all students, with particular attention to those at highest risk for peer victimization in the school context.

By addressing the third aim, this research contributes to our knowledge base regarding pathways leading to psychosocial distress by determining the extent to which exposure to peer victimization, a form of social oppression, contributes to heightened psychosocial distress for youth by disability status; as well as the degree to which sex moderates the mediated effect of peer victimization on psychosocial distress. Interventions arising from this knowledge can incorporate an "upstream" approach that includes a focus on reducing exposure to peer victimization. Research exploring exposure to peer victimization as a predictor of psychosocial health among YwD also may spur researchers to include environmental stressors and protective factors, which are crucial in promoting health. Additionally this research encourages the inclusion of disability as a demographic variable in studies of peer victimization among youth overall.

In addressing all three aims, this research reframes "disability" as a marker of "difference," which increases exposure to peer victimization. This reframing contradicts the persistent medical model in public health and social work research. Methodologically, this research also offers an example of how to do quantitative intersectional analysis using an inter-categorical approach (McCall, 2005; Sen, Iyer, \& Mukherjee, 2009).

\section{Overview of Dissertation}

This dissertation begins with an analysis of the literature in Chapter Two, examining the problem of psychosocial distress and peer victimization among YwD, including research on other marginalizing characteristics, such as sex and sexual 
orientation status, which may intersect with disability status to increase risk for victimization. Chapter Three begins with theoretical antecedents of peer victimization, such as stigma, prejudice, and oppression, and intersectionality before delving into theories related to non-dominant culture status and social location. Minority stress theory, connecting exposure to stress and discrimination and their impact on psychosocial health is reviewed. Chapter Three ends with the study's research questions and hypotheses. Chapter Four describes the research methodology, analysis plan, and strengths and limitations of the study. The results of this study are detailed in Chapter Five. Chapter Six provides a discussion of the primary findings, strengths and limitations, implications for practice, policy and research, and recommendations for future research, and ends with a brief conclusion. 


\section{CHAPTER II: LITERATURE}

There are two sections in this literature review which corresponds to the aims specified in the previous chapter. To help guide the reader, the chapter begins with an overview of key concepts and inclusion criteria relevant to the empirical literature review. Following the overview, the first section focuses on what is known about the prevalence of peer victimization among YwD and other status groups (sexual orientation, race/ethnicity, sex); as well as the intersections of disability among those groups in influencing exposure to peer victimization. This review makes the case that research is needed to examine the prevalence of peer victimization among $\mathrm{YwD}$, alone and in combination with other status factors. Section two of the literature review provides an overview of the problem of psychosocial distress among youth with disabilities (YwD), followed by a review of studies examining the relationship between peer victimization and psychosocial distress. Due in part to the relative paucity of data linking exposure to peer victimization with psychosocial distress among YwD, I also provide a brief overview of the empirical research pertaining to the associations of peer victimization and psychosocial distress in other marginalized populations. Gaps in the empirical literature explaining the relationship between psychosocial distress and peer victimization among youth with disabilities are highlighted throughout this chapter. 


\section{Key Concepts and Inclusion Criteria}

\section{Disability}

This review includes studies involving youth with physical, sensory, cognitive, or learning disabilities with their associated activity limitations. Evincing a historical and persistent connection to a "medical model," the research literature often subsumes disability under the term "chronic condition" or" chronic illness." Many studies pertaining to $\mathrm{YwD}$ in the review either focused on youth with specific impairments or conditions, or used a "noncategorical" approach that included youth with a variety of disabilities with functional limitations. Research applying a noncategorical approach emphasizes the commonality of experience across disability types (Gortmaker et al., 1990; Stein \& Jessop, 1982; van der Lee, Mokkink, Grootenhuis, Heymans, \& Offringa, 2007; Wolman et al., 1994). Such an approach makes it "possible to begin to learn more about characteristics, attitudes, and behaviors of the affected children in relation to the total child population in given communities" (Stein \& Jessop, 1982, p. 361). I used the term "YwD" and "non-disabled" as generic terms reflecting social statuses by disability. When referring to a study, other specific terms or "labels" may be used in order to provide greater specificity relevant to the study population.

Criteria for review. This review included studies that reference both youth with disabilities and youth with "chronic conditions" or "chronic illness," as long as the referent group included youth with disabilities with associated activity limitations. Youth with medical conditions, such as asthma, diabetes, and epilepsy, without activity limitations were not included. Youth identifying as having "an emotional condition such 
as depression or anxiety" (Oregon Department of Human Services, 2008) were excluded as psychosocial distress is positioned as an outcome variable in this study. As the foci of interest in this study is on the experience of peer victimization among youth with disabilities, compared to non-disabled peers in typical settings, I included the empirical findings focused upon youth with intellectual disabilities who were not in self-contained classrooms or separate schools.

\section{Peer Victimization}

There is great variation in defining peer victimization. Some researchers may consider "bullying" and "teasing" as separate behaviors (see for example: Espelage et al., 2008; Kosciw et al., 2010), while others combine the two (Sweeting \& West, 2001). In

general, types of peer victimization can be categorized either as direct or indirect; direct forms of peer victimization, such as being threatened or pushed, can be distinguished from indirect forms of victimization by whether or not there is a face-to-face encounter between the victim and the perpetrator. See Table 1 for a typology of various types of victimization referred to in the empirical literature in this chapter. 
Table 1

Types of Peer Victimization

\begin{tabular}{|c|c|c|}
\hline Indirect (covert) & Direct (overt) & Either direct or indirect \\
\hline $\begin{array}{l}\text { Relational: } \\
\text { - Social exclusion } \\
\text { (e.g., deliberately } \\
\text { being ignored or } \\
\text { left out) } \\
\text { - Rumor spreading }\end{array}$ & $\begin{array}{l}\text { Verbal: } \\
\text { - Teasing, name-calling, made } \\
\text { fun of (sometimes referred to } \\
\text { as "emotional") } \\
\text { - Threatened (e.g., with weapon) } \\
\text { - Harassment } \\
\text { Physical: } \\
\text { - Physical aggression (e.g., } \\
\text { pushing, shoving) } \\
\text { - Physical assault } \\
\text { - Sexual assault }\end{array}$ & $\begin{array}{l}\text { Relational: } \\
\text { - Social exclusion } \\
\text { Other: } \\
\text { - Internet / Cyber- } \\
\text { bullying } \\
\text { - Property damage / } \\
\text { items stolen }\end{array}$ \\
\hline
\end{tabular}


For the purposes of this dissertation study, the term "peer victimization" is used; other terms such as "bullying" or "harassment" are used when referring to a specific study utilizing such terminology. Additionally, while often victims of peer victimization also victimize others, unless otherwise stated explicitly, exposure to peer victimization is meant to refer to those victimized.

Criteria for review. Studies referring to any of the following forms of peer victimization were included in this review: relational victimization (such as being excluded from a social group, target of rumors/gossip), verbal victimization, physical victimization, and cyber victimization (Wang, Iannotti, \& Nansel, 2009).

\section{Psychosocial Distress}

"Psychosocial distress" is used to indicate individual level negative affective states such as depression, anxiety, and suicidality. A variety of instruments were used in the studies reviewed, including those designed to measure mental health outcomes of non-clinical populations, including youth, using measures such as the Mental Health Inventory (MHI-5 based on the SF 36), the Hopkins Symptoms Checklist (SCL), and other instruments normed on clinical populations, such as the Beck Depression Inventory. Theoretical details, particularly in regards to the distinction between mental health outcomes and stress appraisal and coping mediators will be discussed in Chapter Three.

Criteria for review. Studies including negative affective mental health states, such as depression, anxiety, and suicide ideation were included. Measures of suicide attempts also were included because suicide has been more commonly associated with peer victimization experienced by LGBTQ youth in the research literature, as opposed to 
depression and anxiety (Friedman, Koeske, Silvestre, Korr, \& Sites, 2006; Hershberger, Pilkington, \& D'Augelli, 1997; Lewis, 2009).

\section{Intersections and Intersectionality}

Many youth with stigmatized identities (e.g., female, LGB, disabled), experience peer victimization. However, it would be misleading to imply that youth with these various identities experience oppression similarly (Young, 1990). An intersectional approach strengthens our understanding of the degree to which multiple identities affect exposure to peer victimization. This approach entails multiple comparisons of peer victimization among youth with disabilities across other axes of diversity, such as race/ethnicity, sexual orientation, as well as sex. This approach might suggest a question such as whether exposure to peer victimization among disabled lesbians is greater than that of non-disabled heterosexual females or disabled gay/bisexual males. Taking an intersectional approach, it is not sufficient to compare youth with disability by sex, or compare youth with disabilities to non-disabled youth. Indeed, implicit in intersectional research is an assumption that two or more social identities are included in the research (Purdie-Vaughns \& Eibach, 2008). This research study examined the intersections of disability status with other social identities in explicating the magnitude of exposure to peer victimization. Further discussions of theoretical associations between peer victimization and oppression are discussed in Chapter Three.

Criteria for review. This review included research pertaining to adolescent males and females who also may or may not be disabled, LGB, or white, particularly in the context of intersectionality and exposure to peer victimization. 


\section{Other Inclusion Criteria}

Other inclusion criteria were in regards to age; empirical research relating to peer victimization and/or psychosocial distress involving youth ages 11 to 21 was included. A few studies included reported a mean age of youth as less than 11 years, as the sample may have included younger youth as well as older youth. Exceptions were made for retrospective studies in which adults recalled experiences of peer victimization in their youth, and studies in which the age range started in the teen years and extended past age 21.

\section{Section I: Associations and Intersections of Disability, Sex, Sexual Orientation, and Race/Ethnicity with Peer Victimization}

The purpose of this section is to inform the first two aims presented in Chapter One, which were to examine the extent to which disability status is associated with exposure to peer victimization, and taking an intersectional approach, investigate the extent to which disability status in combination with two sets of identity variables (sexual orientation, sex; and race/ethnicity, sex) is associated with exposure to peer victimization.

There are two common types of peer victimization measures: a definition-based measure and a behavior-based measure. This is relevant as the manner in which peer victimization is measured makes a difference in distinguishing group differences, such as those based upon sex and race/ethnicity. In a definition-based measure, typically all questions are preceded with an explanation such as the following: "[bullying is] when a person or group of people repeatedly say or do mean or hurtful things to someone on purpose. Bullying includes things like teasing, hitting, threatening, name-calling, 
ignoring, and leaving someone out on purpose" (Sawyer et al., 2008, p. 108). In a behavior-based measure, questions are usually phrased as follows: "...has someone repeatedly tried to hurt you or make you feel bad by [behavior]..." (Sawyer et al., 2008, p. 109). The differences in prevalence by type of measure is striking, as seen in the study by Sawyer and colleagues (2008), conducted in 2006, which involved 24,345 students from grade 4 to 12, from 107 schools in a Maryland. Overall, 63.9\% of the students identified as white, $17.2 \%$ as black/African American, $4.3 \%$ as Hispanic/Latino, 3.2\% as Asian/Pacific Islander, and $11.35 \%$ as "other" (Sawyer et al., 2008). In this study, the rate of peer victimization among females in high school ranged from $12-28 \%$ using a definition-based measure, and $60-66 \%$ via a 10 -item behavior-based measure that allows for categorizing types of peer victimization by verbal, physical, and indirect (e.g., relational, exclusion). Among males in high school, the percentage of peer victimization ranged from $20-34 \%$ via the definition-based measure, and $54-65 \%$ via the 10 -item behavior-based measure. The behavior-based measures yielded much higher rates of peer victimization, and this difference is thought to be a product of greater specificity regarding the nature of peer victimization assessed, perhaps due to less social desirability bias (Sawyer et al., 2008).

The remainder of this section reviews research on the prevalence of peer victimization by disability, race/ethnicity, sexual orientation, and their intersections, including the crosscutting influence of sex. As will be shown, LGB youth, and in some studies, Latino and multi-racial youth share a commonality with YwD. In both groups, 
there is significantly greater exposure to violence, and specifically, peer victimization, compared to their white, heterosexual, and non-disabled peers.

\section{Disability}

The body of empirical literature described below consists of diverse studies, ranging from studies employing nonrandom samples with control groups, to others utilizing population-based surveys, to some focusing only on specific groups of youth with disabilities, such as mobility disabilities or ADHD. This section begins with a review of the prevalence and frequent types of peer victimization among youth with disabilities. Several multivariate studies examining associations of peer victimization with other predictors among youth with disabilities are examined, and this section concludes with findings from studies examining peer victimization among youth with disabilities by sex.

Studies consistently report higher rates of peer victimization for $\mathrm{YwD}$, compared to their non-disabled peers (Table 2). A study based upon the National Survey of Children's Health 2003-04 data set (YwD $n=12,488$; non-disabled $n=43,963$ ) reported the highest population-based prevalence rate $(52 \%)$ among YwD (van Cleave \& Davis, 2006). This high rate was based upon parents answering affirmatively to the question if they were concerned "their child being 'bullied' by a classmate" (and not concerned about their child "bullying" others) (van Cleave \& Davis, 2006). Parents of disabled children (52\%) were more likely to report being concerned about their child being bullied, compared to parents of non-disabled children $(32.4 \%)(O R=1.46)$. 
Another study, using the WHO Health Behaviour in School Children Survey (HBSC), investigated and compared rates of peer victimization (measured as at least one time in the past couple of months) among three groups in Ireland and France (Sentenac et al., 2011). In addition to a comparison group of non-disabled youth $(n=9778)$, there were two groups of youth with disabilities, one with restrictions in participation at school ( $n=480)$, and another without restrictions in participation $(n=1546)$. YwD with participation restrictions, as well as YwD without restrictions, were significantly more likely to report being victimized, compared to their non-disabled peers, $(O R=1.8[1.4$, $2.4], O R=1.3[1.1,1.4]$ respectively) (Sentenac et al., 2011).

Studies utilizing convenience samples, focusing on youth with specific types of disabilities, also have found significantly higher rates of peer victimization among youth with disabilities, compared to non-disabled youth. For example, in a study which involved youth recruited through two clinics within the same hospital, youth with visible mobility-type disabilities $(n=46)$ reported significantly higher rates of victimization experienced weekly, compared to youth with non-visible health issues (e.g., headaches, asthma) $(n=57)(49 \%$ vs. $20 \%$ respectively, $\mathrm{p}=.004)$ (Dawkins, 1996) (Table 2). In another study involving middle school youth $(N=1315), 34 \%$ of the youth with ADHD (and using medications for ADHD, representing 13.7\% of the study sample) reported being bullied two or three times a month, compared to $22 \%$ of youth not diagnosed as having ADHD or not using medications for ADHD (Unnever \& Cornell, 2003) (see Table 2 for sample details). 
Two studies have examined the types of peer victimization reported by youth with disabilities. Dawkins (1996), referred to above, found that among those with disabilities reporting peer victimization, 59\% reported being called nasty names and 50\% reported being physically hurt. In a study conducted by Carroll and Shute (2005), which focused upon youth with $(n=85)$ and without craniofacial disabilities $(n=55)$, findings included significant differences in experiencing craniofacial aggression at least weekly (i.e. point, stare, snigger, impersonate) between the youth with craniofacial disabilities and the control group $(O R=3.4[1.30,8.76])$.

Several studies employed multivariate analyses to control for other variables in order to determine the extent to which disability status was associated with peer victimization. In the study referred to above that used logistic regression, Dawkins (1996) did not find disability status to be significantly associated with peer victimization. Instead, Dawkins found that playing alone was the strongest predictor of peer victimization; those victimized were more likely to report playing alone, compared to those not reporting peer victimization $(O R=4.0[1.28,12.51])$. In the Unnever \& Cornell (2003) study involving youth with ADHD (reviewed above), regression analyses, controlling for sex, race, height, weight, and other demographic variables, found that taking medications for ADHD $($ beta $=.13, \mathrm{p}<.001)$, being white $($ beta $=-.06, \mathrm{p}<.01)$, and being overweight (beta $=.07, \mathrm{p}<.05$ ), were significant predictors of exposure to peer victimization.

In regards to the intersections of disability and sex, many studies examining prevalence of peer victimization among YwD did not report possible sex-based 
differences between disabled males and females, nor did they contrast those sex differences with their non-disabled counterparts. However, a few studies suggested sex differences. For example in the Health Behaviour in School Children Survey (HBSC) study conducted in Ireland and France, $41 \%$ of disabled females and males in France reported peer victimization, compared to $33.9 \%$ of non-disabled females, and $31.8 \%$ nondisabled males respectively (Sentenac et al., 2011). A similar gradient pattern by disability and then sex can be seen from the rates of peer victimization among the Irish students as well, albeit at lower percentages (Sentenac et al., 2011) (Table 2).

Studies utilizing convenience samples of youth by specific impairments also revealed a similar disability/sex gradient pattern. Results from a study which involved 40 boys and 12 girls all with ADHD diagnosis, and a comparison group of youth without ADHD diagnosis or symptoms, revealed a disability and sex gradient in terms of mean scores from the Bully/Victim Questionnaire (BVQ), which utilizes a 4-point Likert scale to assess frequency of bullying behaviors inflicted on others, as well as peer victimization experienced (Wiener \& Mak, 2009). Significant sex by ADHD status interactions were observed $[F(1,103)=4.63, \mathrm{p}=.034]$. The lowest mean level of victimization was observed among females without $\operatorname{ADHD}(M=8.5, S D=1.98)$, followed by males without $\operatorname{ADHD}(\mathrm{M}=10.35, \mathrm{SD}=2.86)$, then males with $\mathrm{ADHD}(M=13.10, S D=5.28)$; females with ADHD were observed to have the highest means of victimization $(M=15.5$, $S D=5.65)$

As seen from this brief review, youth with disabilities are more likely to report peer victimization compared to their non-disabled peers. Further, females with disabilities 
are more likely to report peer victimization, compared to females without disabilities, as well as males with disabilities. These findings suggest that disability may be a primary determinant of peer victimization when examining sex differences both between and within groups. Little is known, however, about the type and frequency of peer victimization most likely to be experienced by youth with disabilities. The Carroll and Shute (2005) study is remarkable in that they distinguished cranio-specific aggression from other forms of aggression. Among the two multivariate studies examined in this section, the Dawkin's study (1996) found social isolation (playing alone) to be a stronger predictor than disability status, suggesting that disability by itself may not be a useful predictor of exposure to peer victimization. More research situating disability status as a demographic characteristic is needed to investigate socio-environmental predictors of peer victimization, particularly multivariate studies utilizing population-based datasets. Next, I will discuss what is known about sex differences in peer victimization rates among the general population. 


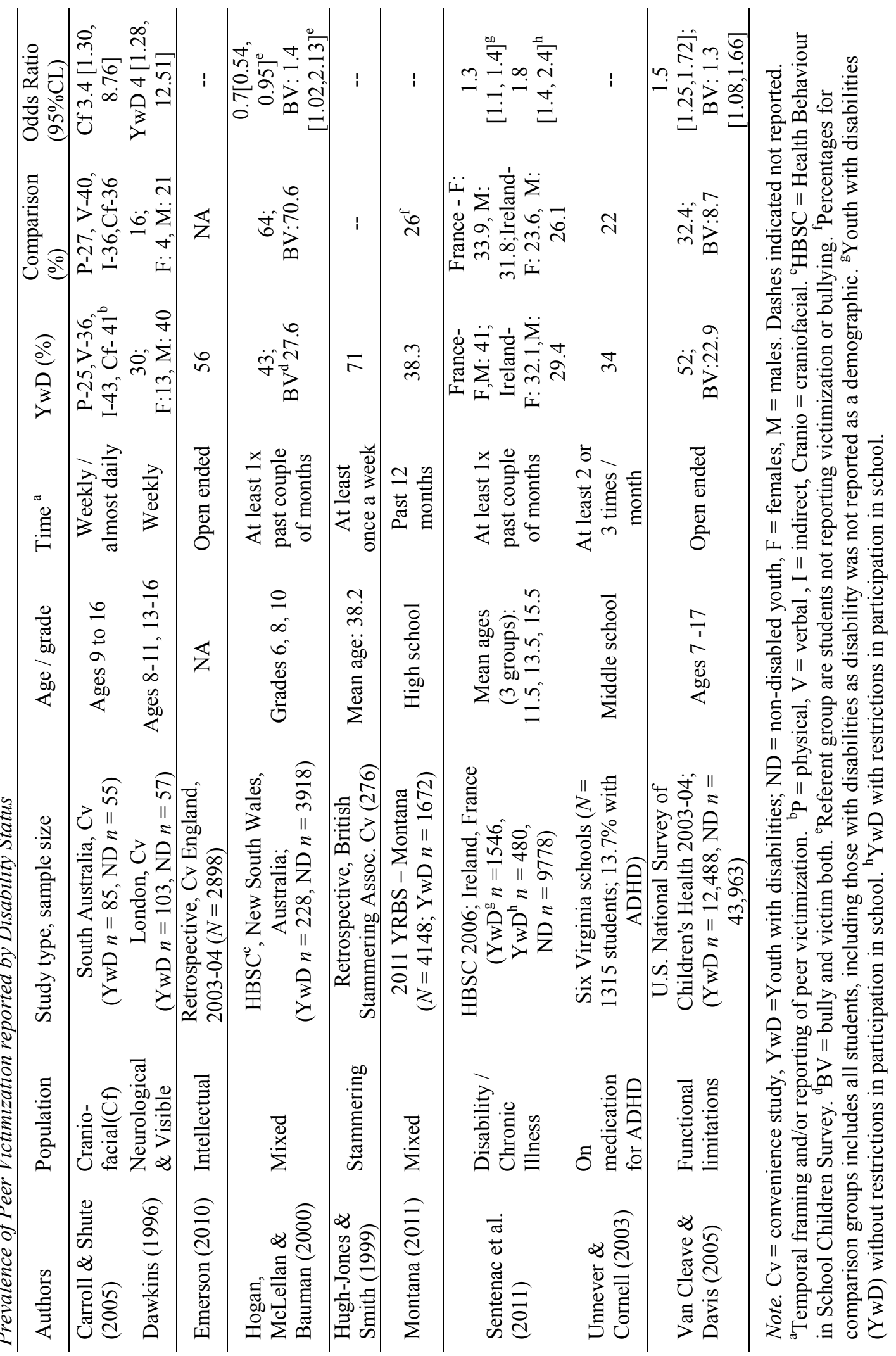




\section{Sex}

This section begins with a clarification on the differences between sex and gender identity, and then provides a review of the prevalence of peer victimization both by sex, and within sex. Before proceeding further, it is important to understand how sex is different from gender identity in this paper. "Sex" refers to biological sex; gender identity is used when the study in question specifically distinguishes gender identity from biological sex. This distinction is important, for example, as an individual with male genitalia may identify as female, or transgender, which would differ from the "apparent" biological sex of the individual at birth. This distinction is particularly salient in the body of work involving LGBT youth. For example, the Gay, Lesbian and Straight Education Network (GLSEN) uses the term "gender identity," providing students with "transgender" and "other gender" options in addition to male and female (Kosciw et al.,2010). The studies examined in this section refer to the apparent biological sex. Studies examining intersections of gender identity and peer victimization (such as the GLSEN climate studies) appear to be limited to studies also examining intersections of sexual orientation and peer victimization, and thus will be covered in the next section below.

Findings are mixed in regards to sex differences. For example, a report based upon results from the 2009 Youth Risk behavior Survey (YRBS), a cross-sectional representative sample of high school students, indicates that a higher percentage of female students $(21.2 \%)$, compared to male students (18.7\%), report being bullied on school property in the last 12 months (no significance information provided) (Eaton et al., 
2010). This may be a function of how peer victimization is defined and the different types of peer victimization (Nabuzoka, Rønning, \& Handegård, 2009; Sawyer et al., 2008). For example, referring back to the YRBS study, a greater percentage of males (15.1\%) reported that they were in a physical fight on school property, compared to females (6.7\%) (Eaton et al., 2010). However, is that is not clear if being in a fight on school property means being victimized, or if it means one was victimizing the other person, or both.

Variations in sex between males and females, and by type of measure, as found in the study by Sawyer and colleagues (2008), support the notion of differences in types of victimization by sex. Specifically, lower rates of peer victimization were found among females (12-28\%) and males (20-34\%) in high school using a definition-based measure; these rates jumped considerably (females, 60-66\%; males 54-65\%) when high schools were queried using a behavior-based measure with 10 items. Interestingly, there are greater differences between high school males and females using the definition-based measure, compared to the behavior-based measure. This may be due to greater specificity in the behavior-based measure, which also includes various types of victimization, such as physical, verbal, and indirect forms of victimization. This can be seen in the study conducted by Sawyer and colleagues, as greater percentages of high school males reported physical forms of peer victimization (34-42\%), compared to high school females $(12-28 \%)$. Further, in regards to indirect forms of victimization (relational, exclusion, cyber), female high school students reported higher rates (40-53\%) compared to male high school students (31-42\%) (Sawyer et al., 2008). The limitation of this research is the 
lack of testing for sex differences. Instead, the focus of the study was upon racial and age differences in peer victimization among males and females separately. Nonetheless, these findings, suggesting sex differences by types of peer victimization experienced, are consistent with another study conducted by Dao and colleagues (2006). That study involved $7^{\text {th }}$ graders (mean age 12.3), and males $(n=86)$ reported more physical and property victimization compared to females $(n=100)$. Females, however, reported more emotional peer victimization than boys (Dao et al., 2006).

Nabuzoka, Rønning, and Handegård (2009) hypothesized that if a robust definition of peer victimization included relational and physical forms was used, one would not find sex differences in rates of peer victimization. Thus, their study with high school students $(N=575)$, ages 11 to 15 , used an inclusive standard definition of peer victimization, collapsing various types of peer victimization. However, their results refuted their hypothesis, as female students $(68.4 \%)$ were still significantly more likely to report exposure to peer victimization compared to their male counterparts $\left(56.4 \%, X^{2}(1)\right.$ $=3.84, \mathrm{p}<.05$ ), indicating that sex differences may not be simply due to how the peer victimization questions are constructed.

As seen above, the current research in regards to sex differences is indeterminate. The findings reported by Nabuzoka, Rønning, and Handegård (2009) do not support the notion that sex differences in exposure to peer victimization may be more of a matter of type of victimization (e.g., relational versus physical); it may be that females do experience more peer victimization than males. Clearly more research is needed to 
determine how sex differences influence both the type of peer victimization experienced, as well as the prevalence of such.

\section{Sexual Orientation}

This section begins with a review of the prevalence and frequent types of peer victimization among LGB youth. I then review the empirical literature pointing to sex differences in peer victimization rates among youth by sexual orientation status. The reader may notice that this body of literature is relatively robust compared to those studies examining differences by either disability status or sex.

Peer victimization rates among LGBT youth in the peer reviewed literature are high. A study conducted by Gruber and Fineran (2008), which included 522 students from middle and high schools, found that LGBQ students were more likely to report being bullied (79\%) and sexually harassed (71\%) in the past year, compared to their peers who identified as heterosexual ( $50 \%$ and $32 \%$ respectively) (beta coefficients were .217 and .28 respectively, $\mathrm{p}<.01$ ) (Table 3 ). Gruber and Fineran (2008) defined students as "bullied" if they reported experiencing three or more types of peer victimization (e.g., being teased, pushed and excluded) in the past year.

Higher rates of peer victimization among LGB youth also were found in a metaanalysis conducted by Friedman and colleagues (2011), which examined 26 school-based studies (including unpublished Youth Risk Behavior Survey studies, but excluding convenience studies) in Canada and the U.S. conducted between 1980 and 2009 among junior and high school students (most included students in grade 9 to 12). In this metaanalysis, the authors defined "assault by peers" as being threatened with a weapon, or 
being assaulted or injured at school. Differences in exposure to peer victimization/assault by peers between LGB students and their heterosexual peers were significant, with effect sizes across the 26 studies ranging from 2.59 to 2.71 (mean effect size across all studies $=$ $2.68, O R=2.7[2.4,2.98], \mathrm{p}<.001)($ Friedman et al., 2011). Interestingly, the dimensions used to categorize sexual orientation status (sexual behavior, romantic attractions and self-identification) were not found to be a significant moderator of the association between sexual orientation and peer victimization/assault (Friedman et al., 2011). In other words, the relationship between sexual orientation and peer victimization did not significantly differ based upon whether studies used sexual identity questions (and/or romantic attractions), or used sexual behavior (by sex of partner) to categorize as LGB. In addition, it is notable that the decade in which the survey was administered (in the 1990's, compared to the 2000's) did not moderate the association between sexual orientation and peer victimization (Friedman et al., 2011), suggesting that peer victimization rates among LGB youth have not significantly decreased over the last 10 years.

The National School Climate Studies conducted by the Gay, Lesbian and Straight Education Network (GLSEN) provide a level of detail seldom seen in this body of research (Kosciw et al.,2010). Over seven thousand LGBT youth were recruited in 2009 through randomly selected community based groups $(N=7261$, mean age $=16.3)$, as well as through their web-based networks (e.g., emails, listservs) (Kosciw et al.,2010). The gender diverse sample $[57.1 \%$ female, $33.2 \%$ male, $5.7 \%$ transgender, and $4 \%$ other 
gender (e.g., genderqueer)], ${ }^{4}$ also was somewhat ethnically and racially diverse $(67.4 \%$ white, $14.1 \%$ Latino/a, $4 \%$ black, 2.6\% Asian/Pacific Islander, $0.5 \%$ Native American/Alaskan Native, and $10.4 \%$ multiracial). Nearly two thirds of the sample (61\%) identified as gay or lesbian, $31.6 \%$ identified as bisexual, $4.5 \%$ identified another type of sexual orientation (such as queer, pansexual), and 3\% identified as questioning or unsure (Kosciw et al.,2010). The authors captured homophobic remarks as a separate category, in addition to exposure to verbal, physical harassment and physical assault. Thus, while $90 \%$ of the sample reported name calling, or being threatened (verbal harassment) in the past year, $72.4 \%$ of LGBT students also reported hearing homophobic remarks (e.g., "no homo," "fag") "frequently" or "often" while at school in the past year. Sexual orientation and gender expression were given as the top two reasons cited for experiencing verbal harassment, physical harassment or physical assault (Kosciw et al.,2010) (Table 4). Excluding students who identified as questioning or "unsure," verbal harassment due to sexual orientation was the most common form of peer victimization reported among all LGBT students, with percentages ranging from $58.4 \%$ for bisexual youth, to $69.7 \%$ for lesbian or gay youth, followed by physical harassment (20.3\% to $38.8 \%)$, and physical assault (8.8\% to $16.5 \%)$ (Table 4$)$. The experience of relational aggression was also high, with $88.2 \%$ of LGBT students reporting social exclusion, and $84 \%$ reporting being a target of rumors or lies; over $40 \%$ of the students reported these forms of relational victimization "frequently" or "often." Over half of the students (52.9\%) experienced

\footnotetext{
${ }^{4}$ See http://internationalspectrum.umich.edu/life/definitions or www.glma.org/_data/n 001/resources/live/HealthyCompanionDoc3.pdf for a glossary of terms commonly associated with sexual and gender minority people.
} 
cyber-bullying in the past school year, with $14 \%$ reporting that it occurred "frequently" or "often." More than two-thirds of the students (68.2\%) reported experiencing sexual harassment (e.g., unwanted touching, sexual remarks) in the past year, with $20.8 \%$ reported it happening "often" or "frequently" (Kosciw et al., 2010).

The GLSEN report also indicated the percentage of students reporting verbal harassment, physical harassment and physical assault in the past year, by sexual orientation status (including questioning/unsure), as well as noting the reason students attributed to being victimized due to sexual orientation status (Kosciw et al., 2010) (Table 4). The differences between the groups are striking, with 69.7\%, 28.1\%, and 1.1\% of lesbian/gay students reporting verbal harassment, physical harassment and physical assault, frequently or often, respectively, in contrast to $38.2 \%, 9 \%$, and $0.09 \%$ of questioning/unsure students. It is likely that the questioning students are not "visible" targets. Analyses using MANOVA documented significant differences across the categories $(\mathrm{p}<.001)($ Kosciw et al., 2010).

The meta-analysis conducted by Friedman and colleagues (2011), mentioned above, also evaluated whether sex significantly moderated the relationship between sexual orientation and peer victimization/assault, which it did $(\mathrm{p}<.001)$. While gay or bisexual males were more likely than heterosexual males to report peer victimization/assault $(O R=2[1.68,2.46])$, their odds of victimization were lower than those of lesbian or bisexual females, who also were more likely than heterosexual females to report peer victimization $(O R=3.3[2.82,3.89])$ (Friedman et al., 2011). Interestingly, results of the meta-analysis indicated that the percentage of gay males 
reporting peer victimization (43.2\%) did not vary much from the percentage of lesbians reporting exposure to victimization (44.5\%). Rates of peer victimization/assault did, however, vary for those identifying as bisexual; bisexual males $(50.2 \%)$ were more likely to report peer victimization/assault, compared to bisexual females $(39.9 \%)$.

This patterning of peer victimization rates with sexual orientation status primary, followed by sex, can be seen in also in a study conducted by Berlan and colleagues $(N=$ 7559 , mean age $=17$ ), in which lesbians were more likely to report peer victimization compared to heterosexual females $(R R=3.36$ [1.76-6.41] $)$, and gay males were more likely to report experiencing peer victimization, compared to heterosexual males $(R R=$ $1.98,[1.39,2.82])$ (Berlan et al., 2010). The results from the GLSEN study mentioned above also suggest that lesbian/bisexual females and transgender and "other gender" students are significantly less likely to report peer victimization due to sexual orientation, compared to male students identifying as gay or bisexual, as well as youth who identify as transgender and "other gender" ( $\mathrm{p}<.001)$ (Kosciw et al., 2010) (Table 4). As the GLSEN survey design allowed students to identity as transgender, it also provides more information on variations by gender identity, suggesting that transgender students are at greater risk of peer victimization compared to male, female and "other gender" students. The differences by gender identity are particularly evident when it comes to physical harassment and physical assault, with $38.3 \%$ and $15.8 \%$ of transgender students reporting physical harassment and physical assault compared to $27.3 \%$ and $10.3 \%$ of male students, and compared to $21.3 \%$ and $9.8 \%$ of female students respectively (Table 4 ). 
Thus, LGB students are more likely report peer victimization, compared to their heterosexual peers. Two studies specifically asked about homophobic teasing (Espelage et al., 2008; Kosciw et al., 2010), which provided additional information about the type of verbal harassment experienced by students. Sexual orientation status consistently appears to be a primary determinant of exposure, followed by sex. In other words, while both male and lesbian or bisexual female students were more likely to report peer victimization, compared to their heterosexual counterparts, the prevalence of peer victimization tended to be highest among gay and bisexual males, followed by lesbian and bisexual females, and lowest among heterosexual females (Berlan et al., 2010; Friedman et al., 2011; Kosciw et al., 2010). Students who identified as "questioning” or "unsure" were less likely to report verbal, physical harassment, and physical assault, compared to students who identified as LGBT (Kosciw et al., 2010), suggesting perhaps that the questioning students are not "visible" targets. 





Table 4

Percentage of LGBT Students Reporting Type of Peer Victimization by Sexual orientation and Sex/Gender Identity

Sexual orientation as reason for victimization

\begin{tabular}{lccc} 
& \multicolumn{3}{c}{ Sexual orientation as reason for victimization } \\
\cline { 2 - 4 } & Verbal harassment & Physical harassment & Physical assault \\
\hline Sexual orientation & 69.7 & 28.1 & \\
Lesbian or gay & 58.4 & 20.3 & 12.1 \\
Bisexual & 63.2 & 21.4 & 8.8 \\
Other & 38.2 & 9.0 & 9.5 \\
Questioning/ unsure & & & 0.90 \\
\hline Sex/ Gender Identity & 61.6 & 21.3 & 9.8 \\
Female & 69.1 & 27.3 & 10.3 \\
Male & 67.7 & 29.6 & 12.9 \\
Other gender & 69.5 & 38.8 & 16.5 \\
Transgender & & & \\
\hline
\end{tabular}

Note. Source: Kosciw et al. (2010). 


\section{Race/ Ethnicity}

Research focused on peer victimization by racial and ethnic group identity predominantly fail to find significantly higher rates of victimization among students of color overall, even in samples that are highly diverse by race and ethnicity (Hanish \& Guerra, 2000; Juvonen, Graham, \& Schuster, 2003; Spriggs, Iannotti, Nansel, \& Haynie, 2007; Wang, et al., 2010). This section begins with further discussion of the study by Sawyer and colleagues, described earlier in this chapter (2008, p. 23).

Sawyer and colleagues (2008) sought to determine if one of the reasons students of color seem to report lower rates of peer victimization had to do with differences in the two self-reported measures of peer victimization: the single definition-based measure, and the behavior-based measure. Sawyer and colleagues (2008) theorized that the definition-based measure might produce lower prevalence estimates for either or both of two reasons: 1) social desirability (students may resist the label of being bullied); or 2) not understanding the difference between "bullying" (with a focus on repeated intentional aggression inflicted by someone with more power), and other acts of aggression (such as being in a fight with a classmate who does not have more power). On both the single definition-based measure, and the behavior-based measure, male and female African American students were less likely to report peer victimization, compared to male and female white students. In particular, African American male students compared to white male students, were significantly less likely to report peer victimization (single based measure: $O R=0.60[0.40,0.89])$ (Sawyer et al., 2008). However, their findings, on both measures, revealed significantly higher rates of peer victimization among other high 
school students of color (excluding African American students), compared to white students.

The Sawyer et al. study (2008) further found that Latino and Asian male high school students, compared to their white male peers, were significantly more likely to report bullying via the definition-based measure $(O R=1.7[1.17,2.38]$ and $O R=1.7$ $[1.01,2.76]$ respectively, $\mathrm{p} \leq .05$ ) ( Table 5). With the behavior-based measure, high school males identifying as "other" (race/ethnicity) or Latino were more likely to report peer victimization compared to their white male peers $(65.3 \%, O R=1.6[1.11,2.16]$ and $63.9 \%, O R=1.5[1.07,1.98]$ respectively, $\mathrm{p} \leq .05)$. Additionally, significant differences were found by type of victimization, with Hispanic/Latino high school males reporting more physical $(42 \%, O R=1.39, \mathrm{p}=.003)$, indirect $(56.3 \%, O R=1.57, \mathrm{p}=.017)$, and direct verbal $(41.2 \% O R=1.59, \mathrm{p}=.002)$ forms of peer victimization compared to white high school males (34.6\%, 44.8\% and 30.9\% respectively) (Sawyer et al., 2008). ${ }^{5}$

Among high school females, only those who identified as "other" (race/ethnicity) via the definition-based measure were significantly more likely to report being bullied, compared to white females (Sawyer et al., 2008) (Table 5). Significant differences were found by type of victimization, with Latina high school females reporting more physical $(O R=1.44, \mathrm{p}=.033)$ and indirect $(O R=1.65, \mathrm{p}<.001)$ forms of peer victimization, compared to white high school females (Sawyer et al., 2008).

Differences in peer victimization rates by race and sex also are reflected in results from the 2009 Youth Risk Behavior Surveillance; 15.5\% of black high school female

\footnotetext{
${ }^{5}$ Odds ratios reported without the $95 \%$ confidence levels indicate information not provided in the study.
} 
students surveyed reported being harassed on school property in the past year, compared to $11.9 \%$ of white high school female students (see Table 5) (no significance information included) (Eaton et al., 2010). The percentages of peer victimization reported by high school students in the U.S. by race and sex are summarized in Table 6 below, using the results from Sawyer, Bradshaw and O'Brennan (2008) and Eaton et al. (2010). While both studies by Eaton and colleagues (2010) and Sawyer, Bradshaw and O'Brennan (2008) suggest that black females are at increased risk compared to black males, results from the YRBS study suggest that white males and females were most likely to report peer victimization, compared to students of color (Eaton et al., 2010). The results may be a function of the question used on the YRBS, which asked if the student experienced bullying on school property in the past 12 months

While there may not be unequivocal evidence of higher risk of exposure to peer victimization among a single racial/ethnic group, the findings reviewed above suggest that Asian/Pacific Islanders, Hispanic/Latino students, and students of "other" race/ethnicity may be at increased risk compared to white students (Sawyer et al., 2008). Further, findings suggest that race may be a primary determinant of peer victimization, followed by sex, with female students identifying as Hispanic/Latino or "Other" more likely to report peer victimization than their male counterparts (Sawyer et al., 2008).

Clearly, more studies are needed, with attention to the type of instrument being used to measure peer victimization, and testing for differences by race and sex. The lower rates among African American youth, compared to white youth, may be a function of the racial/ethnic composition of the school, as suggested by a longitudinal study with 


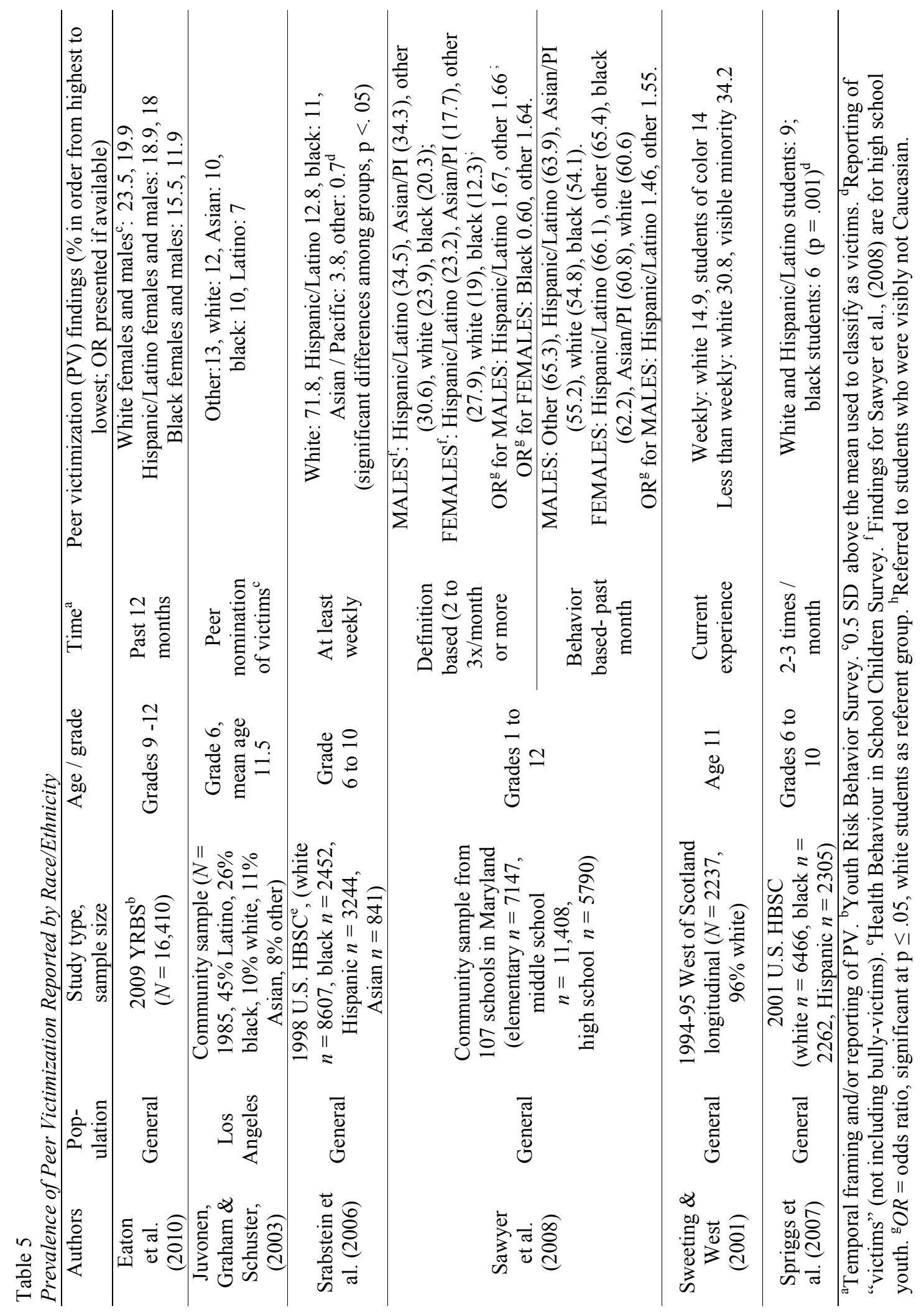


Table 6.

Percentages of Peer Victimization Reported by High School Students by Race, and Sex

\begin{tabular}{lcc} 
& Sawyer et al. $(2008)^{\mathrm{a}}$ & ${\text { Eaton et al. }(2010)^{\mathrm{b}}}^{\mathrm{a}}$ \\
\hline Hispanic/Latino female & $74.0(1)$ & $18.9(3)$ \\
Other female & $65.4(2)$ & -- \\
Other male & $65.3(3)$ & -- \\
Hispanic/Latino male & $63.9(4)$ & $18.0(4)$ \\
Black female & $62.2(5)$ & $15.5(5)$ \\
White female & $60.6(6)$ & $23.5(1)$ \\
White male & $54.8(7)$ & $19.9(2)$ \\
Black male & $54.1(8)$ & $11.9(6)$ \\
\hline
\end{tabular}

Note. Figure in parentheses reflects ordering of frequency from most to least. Dashes indicate data not reported.

${ }^{a}$ Peer victimization measured by behavior-based measure (10-item, in past month). ' 2009 YRBS; peer victimization measured by those reporting being bullied on school property in past 12 months. 
elementary school children (Hanish \& Guerra, 2000). In their study, Hanish and Guerra (2000) found that in schools in which the majority of students were students of color, white students were at greater risk for victimization. Further, African American youth in predominantly African American schools were slightly more likely to be victimized compared to African American youth in predominantly white schools.

\section{Intersections of Disability and Sexual Orientation}

Just as it is important to examine the intersections of sex with disability, sexual orientation, and race/ethnicity, which reveal differential patterns of vulnerability to peer victimization, it is also important to examine patterning of peer victimization rates by the intersections of disability with sexual orientation and race/ethnicity, respectively. Given that there are LGB youth with disabilities, and that the percentage of LGB youth with disabilities may be slightly higher compared to the proportion of heterosexually identified youth with disabilities (Surís, Resnick, Cassuto, \& Blum, 1996), it is an important intersection to consider.

While the GLSEN 2009 study reviewed above did not ask students about their disability status, it did ask students about their experiences with peer victimization due to an actual or perceived disability (Kosciw et al., 2010). The percentages of LGBT students reporting victimization on the basis of an actual or perceived disability was low (17.1\%) compared to other reasons for peer victimization, such as sexual orientation (84.6\%) or gender expression (63.7\%) (Table 7). It is unfortunate that the GLSEN climate survey did not include disability status as a demographic variable that would allow further analyses to see if the proportion of victimization among LGBT disabled students were the same, 


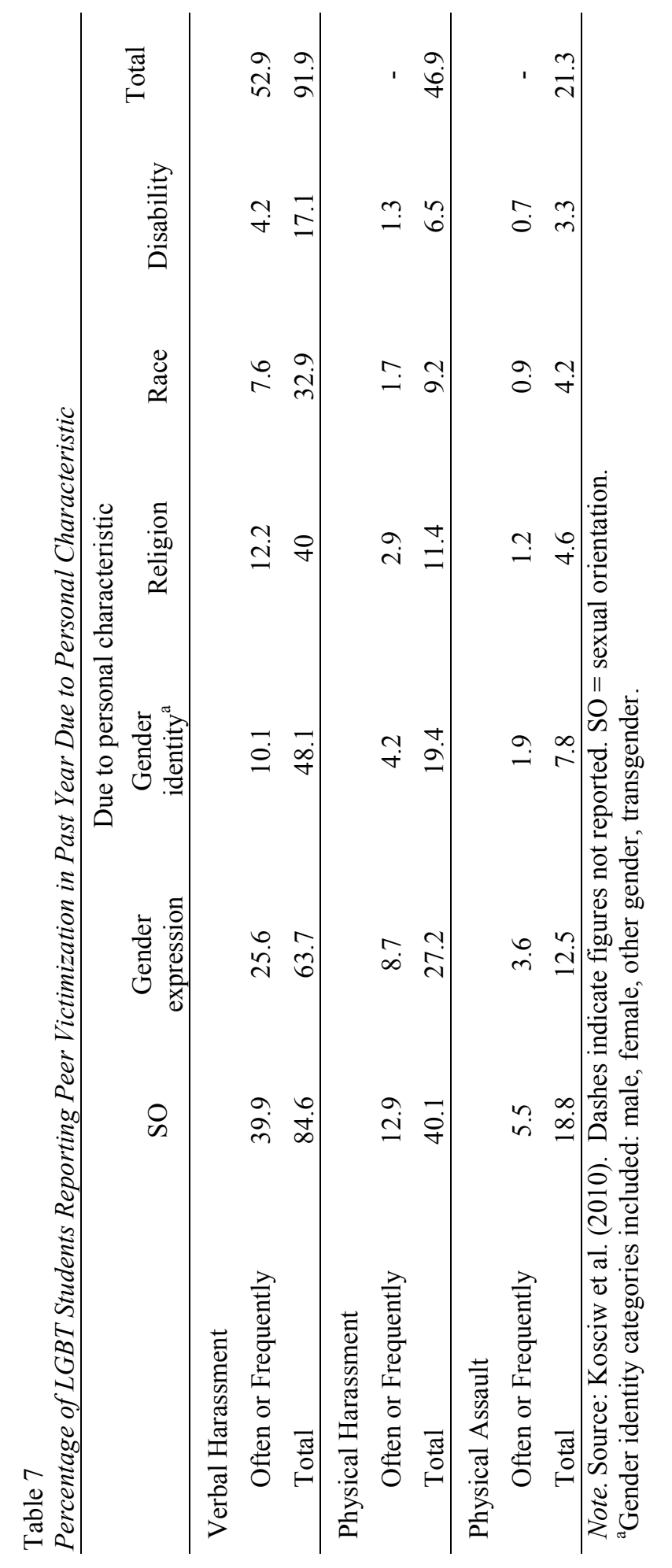


higher or lower compared to non-disabled LGBT students (Kosciw et al., 2010).

Research directly examining exposure to peer victimization among LGBT youth with disabilities was not found.

\section{Intersections of Disability and Race/ethnicity}

There is little research available examining the intersections of disability and race/ethnicity. However, there is some indication that the combination of disability status and non-dominant race/ethnicity statuses may result in increased exposure to peer victimization. This section will rely upon findings from the California Healthy Kids Survey (CHKS) (WestEd \& California Dept. of Education, 2008). While disability status was not collected as a demographic variable, nor was statistical testing information provided, the study did ask about the perceived reason(s) for being bullied or harassed in the past 12 months, including both race/ethnicity and disability. The CHKS study of nearly 700,000 students in grades 7, 9, and 11, included a fairly diverse sample with $45 \%$ of $11^{\text {th }}$ graders identifying as Hispanic or Latino/a, followed by $34 \%$ white (non-Latino), 13\% Asian, 11\% multi-racial, 8\% other, 8\% black/African American, 4\% Native Hawaiian/Pacific Islander, and 3\% American Indian/Alaska Native. Among $11^{\text {th }}$ graders reporting peer victimization in the past 12 months, $21 \%$ reported being pushed, shoved, or hit, $46 \%$ reported sexual jokes/comments/gestures, and 33\% reported being "made fun of because of your looks/way talk." Rates of peer victimization among YwD by other social statuses can only be derived from surveys that ask students to identify the reason(s) they experienced peer victimization, such as race/ethnicity, gender, disability, and sexual orientation. In the case of the CHKS, $15 \%$ and $5 \%$ of $11^{\text {th }}$ graders indicated that the 
reasons for harassment were race/ethnicity/national origin and disability, respectively. Results from an online query database maintained by WestEd (which included students from $7^{\text {th }}, 9^{\text {th }}$, and $11^{\text {th }}$ grade; no significance information given) provided "finer-grained" information in regards to the intersections of disability and race/ethnicity. Students of color, with the exception of Asian and Latina/o students, were more likely to report being harassed or bullied in the past 12 months due to disability, compared to white students. Students were not asked whether they had a disability; they were only asked whether they had experienced harassment due to disability. Native American (8.1\%), African Americans/black (7.6\%), Pacific Islander (7\%), “other” (6.7\%) and Multiethnic (6\%) students all reported at least one incident of peer victimization in the past year due to a disability, compared to white students (5.4\%); Asian (5\%) and Latina/o (4.5\%) students were less likely to report peer victimization in the past year due to a disability, compared to all other races. While these figures are suggestive of differential vulnerability to peer victimization by race/ethnicity and disability, the study itself falls short of being a true intersectional study, as it did not examine the prevalence of peer victimization with disability status as a demographic variable (as it did for race/ethnicity status).

Although evidence of increased peer victimization among students of color compared to their white counterparts is not unequivocal, the intersection of race/ethnicity and disability needs to be considered; further research examining the intersections of race/ethnicity and disability (as a demographic) is warranted. 


\section{Section I Summary: Associations and Intersections}

While some studies reviewed in this chapter have methodological limitations, such as comparing a convenience sample of YwD to a representative sample of nondisabled youth (Huurre \& Aro, 1998; Stevens et al., 1996), and asking about whether students were harassed because of a disability, but not with disability as a demographic variable (Kosciw et al., 2010; WestEd \& California Dept. of Education, 2008), the research is helpful in building knowledge in regards to the association of peer victimization and disability status. There is a need for further research examining the extent to which YwD are exposed to peer victimization, particularly in regards to the intersections of disability, sex, race/ethnicity, and disability, sex, sexual orientation statuses.

There are four conclusions from this literature review. First, there appears to be a consistent pattern of youth with non-dominant culture statuses, whether it is disability, multiracial or "other" identity, Latino ethnicity, or LGB status, reporting greater exposure to peer victimization compared to their counterparts (including students unsure of their sexual orientation). Second, there is relatively little research examining the prevalence of peer victimization among YwD with attention to the intersections of sexual orientation, race/ethnicity. Third, exposure to peer victimization by social status may be influenced by sex as well as race/ethnicity; for example, gay males report more peer victimization compared to lesbians (Berlan et al., 2010), and disabled or black females report more peer victimization compared to disabled or black males (Eaton et al., 2010; Sawyer et al., 2008). Fourth, it is likely that vulnerability to peer victimization among students of color 
is increased with disability status (WestEd \& California Dept. of Education, 2008). The research reviewed above, however, does not suggest either positively or negatively, differential exposure to peer victimization among LGB students by disability status.

\section{Section II: Psychosocial Distress and Peer Victimization}

The previous section contained information about who is at most risk for peer victimization. In this section, the body of empirical literature reviewed connects the impact of exposure to peer victimization with psychosocial distress. However, as the focus of this dissertation research is on disability status, it is important to first examine what is known about the relationship between disability status and psychosocial distress, as well as sex differences by disability status. In order to inform the dissertation research, I also reviewed empirical studies examining the extent to which exposure to peer victimization mediates the relationship between a particular social status (e.g., sexual orientation) and psychosocial distress, followed by studies examining sex as a moderator on the relationship between peer victimization and psychosocial distress.

\section{Psychosocial Distress: Youth with Disabilities}

The literature is consistent in reporting higher levels of psychosocial distress among YwD compared to their non-disabled peers. This section will start with a review of a meta-analysis conducted by Lavigne and Faier-Routman (1992) which involved 87 studies of psychological adjustment by children and youth with physical disabilities. Several studies not reporting higher levels of psychosocial distress among YwD compared to their non-disabled peers will be reviewed, as well as a longitudinal study examining the trajectory of psychosocial distress among youth with spina bifida from age 
8 or 9 , to age 12. I also will review the empirical literature suggesting differences among youth with disabilities by sex with respect to psychosocial distress. I then conclude with a summary of the significance of this section of the review.

The meta-analysis conducted by Lavigne and Faier-Routman (1992) included studies involving children and youth with physical disabilities or conditions, such as cancer, asthma, arthritis, as well as orthopedic impairments, ages 3 to 19. Inclusion criteria for the meta-analysis required study sources to include overall adjustment measures, which could include internalizing (e.g., self-esteem) or externalizing measures, and presentation of the data in such a way that would allow calculation of effect sizes either with a study control group or with normative data. Physical disability status was found to be significantly correlated with psychosocial distress $(\mathrm{p}<.01)$. The mean effect size of overall psychosocial adjustment, in relation to disability status, ranged from .47 to $.78(\mathrm{p}<.01)$ depending on the research design. Studies with a comparison group produced lower mean effect sizes $(.47-.59, \mathrm{p}<.01)$. Studies with matched controls, and those controlling for other variables such as age, sex, SES and race, had higher mean effect sizes $(.60$ to $.78 ; \mathrm{p}<.01)$. Those studies with normative comparisons had the highest mean effect sizes ranging from .73 to .88 . In regards specifically to internalizing symptoms, which included indicators such as anxiety, depression, and social withdrawal, the mean effect sizes ranged from .44 in studies with comparison groups, to .48 in studies with normative comparisons $(\mathrm{p}<.01)$ (Lavigne \& Faier-Routman, 1992). Lavigne and Faier-Routman's (1992) meta-analysis results are in line with other studies examining psychosocial distress among youth with certain impairments (Blum, Resnick, Nelson, \& 
St Germaine, 1991; Holmbeck \& Devine, 2010; Howe, Feinstein, Reiss, Molock, \& Berger, 1993; Tate, Forchheimer, Maynard, \& Dijkers, 1994). Impairment-specific studies included youth with vision loss (Huurre \& Aro, 2000; Koenes \& Karshmer, 2000) and mobility disabilities (Varni \& Setoguchi, 1992; Varni, Setoguchi, Rappaport, \& Talbot, 1991).

While most studies reviewed were consistent in reporting significant differences between YwD and non-disabled youth in regards to psychosocial distress, there were two exceptions (Huurre \& Aro, 1998; Stevens et al., 1996). Both studies involved a convenience sample of students with disabilities and a separate comparison sample of non-disabled students. The first study involved 54 students with vision impairments, and a comparison group of 385 non-disabled students, in grades 7 to 9 in Finland (Huurre \& Aro, 1998). Huurre and Aro (1998) did not find significant differences between students with and without vision disabilities in psychosocial well-being, as measured by a 17 -item checklist of physical and psychological symptoms, a modified Beck Depression Inventory, and a self-esteem instrument. The subsequent study included a much larger sample of students with vision impairments $(n=115)$, and two comparison groups: sighted youth who answered "yes" or "no" to "Do you have any chronic condition or disability that interferes with your daily life?" (sighted youth with disabilities, $n=44$; non-disabled youth, $n=607$ ) (Huurre $\&$ Aro, 2000). In this study, significant differences in distress symptoms measured by a 17 -item checklist were found, with males with vision impairments $(n=76)$ reporting fewer distress symptoms than non-disabled males $(n=$ 275), who in turn reported fewer distress symptoms than males with chronic conditions ( $n$ 
=19) $(F=10.69, \mathrm{p}<.001){ }^{6}$ Likewise there were differences among the females with non-disabled females $(n=332)$ reporting fewest distress symptoms and females with chronic conditions $(n=25)$ reporting the most distress symptoms $(F=3.34, \mathrm{p}=.04)$, number of females in study with visual impairment was 39) (Huurre \& Aro, 2000). As noted by the authors, several limitations included small group sizes, which make it more difficult to detect statistical significance. Further, the sampling of the groups differed (blind youth recruited through a registry while the others were surveyed through a representative sampling design).

The second study utilizing the WHO Health Behaviour in School Children (HBSC) survey in Canada (Stevens et al., 1996) did not find significant differences between youth with and without disabilities in regards to psychosocial health. This study also compared disabled youth ( $n=101$, convenience sample) to that of a national sample of students $(n=7020)$ (Stevens et al., 1996). Decisions on data analysis, as well as sampling, may have contributed to nonsignificant findings, as a study using the same survey instrument in Australia (Hogan, McLellan, \& Bauman, 2000) reported significant findings relating to psychosocial distress. The Australian study found, that after controlling for grade, age, and sex, that youth with disabilities $(n=228)$, compared to their non-disabled peers $(n=3618)$, were more likely to report the following symptoms of psychosocial distress: feeling lonely $(O R=1.7[1.24,2.4])$, isolated $(O R=1.6[1.2$, $2.12])$, feeling low $(O R=1.8[1.35,2.39])$, and nervous $(O R=1.4[1.08,1.88])$ (Hogan et al., 2000). While the differences in findings among the two studies may reflect actual

\footnotetext{
${ }^{6}$ Degrees of freedom not reported. Thus sizes of all subgroups are given.
} 
differences in the populations, it is also possible that research design differences also accounted for the different finding; there were significant differences in study design (representative versus convenience), data collection (assistance with survey including face-to-face versus survey filled out with anonymity), and data analysis approach (i.e. coding responses to the question relating to feeling lonely).

In contrast to the correlational studies covered thus far, a six year longitudinal study with youth with spina bifida $(n=60)$ and a control group of non-disabled youth ( $n$ $=65$ ) matched on ten demographic variables, revealed a reverse trajectory of depressive symptoms for youth with spina bifida (Holmbeck \& Devine, 2010). At the start of the study (with all youth at about age eight or nine), youth with spina bifida started with a higher intercept (0.208) with a negative linear slope (-0.006), which translated into higher mean levels of depressive symptoms compared to their non-disabled controls (intercept $=.172$, slope $=0.007)$. By age 12 , however, both groups reported about the same level of depressive symptoms, and by age 15 youth with spina bifida had lower means levels of depressive symptoms. In discussing these findings, the authors speculated that the children with spina bifida and their families may be "less responsive to the developmental changes of adolescence than are families of typically developing children" (Holmbeck \& Devine, 2010, p. 523); in other words, while the trend was for non-disabled youth to have higher mean levels of depressive symptoms in their teens, the patterning of such was different for youth with spina bifida. 


\section{Psychosocial Distress: Intersections of Disability and Sex}

Results from a population based survey in Switzerland conducted in 1992-93 with 9268 in-school youth, aged 15 to 20 years (Miauton et al., 2003) were suggestive of differences in regards to psychosocial distress by disability and sex. In the study conducted by Miauton, Narring and Michaud (2003), in which 11.4\% of females and 9.6\% of males self-identified as having a physical disability and chronic illness, a higher percentage of females reported "often" feeling depressed compared to males. Disabled females and males reported higher levels of depression $(38.1 \%, 21.1 \%$ respectively), compared to non-disabled females and males (31.1\%, 15.4\% respectively); differences among females $(\mathrm{p}=.003)$ and males $(\mathrm{p}=.001)$ by disability status were statistically significant (Miauton et al., 2003).

A similar pattern can be found in the study conducted by Wolman, Resnick, Harris and Blum (1994), based upon the 1986-87 Minnesota Adolescent Health Survey, involving 1683 disabled and 1650 non-disabled middle and high schools students (Wolman et al., 1994). Their findings suggest a sex-based gradient, in which disability status seems to operate through sex, with sex acting as primary determinant. Specifically, a larger percentage of disabled females reported a high degree of worries about dying soon, and worries about peer relations, followed by non-disabled females, than disabled males; non-disabled males reported lowest levels of high degrees of worry. To give an example, across one of these variables, $61 \%$ of disabled females reported a high degree of worry in regards to peer relations, compared to $55 \%$ of non-disabled females, followed by $47 \%$ of disabled males, and $38 \%$ of non-disabled males. Unfortunately, most studies 
reviewed did not test for sex differences in psychosocial distress by both disability and sex status. Generally the focus was on differences among males, with and without disabilities, and/or differences among females with and without disabilities. For example, significant differences were reported between disabled and non-disabled males in regards to worries about peer relations $\left(X^{2}=21.32, \mathrm{p}<.001\right)$, whereas no significant differences were found between the disabled and non-disabled females (Wolman et al., 1994). But we don't know if there were significant differences by both disability and sex in regards to worries about peer relations. Nonetheless, psychosocial distress does seem to be higher among females compared to males, and higher among disabled youth compared to nondisabled youth.

This type of patterning, with psychosocial distress higher among females compared to males, and higher among disabled youth compared to non-disabled youth, does not always hold true. Significant differences both by sex and disability status were observed in a convenience sample involving three groups of youth ages 12 to 18: youth with neurological conditions ( $n=80,37 \%)$, youth with non-neurological disabilities (e.g., vision, diabetes, cerebral palsy) $(n=85,40 \%)$, and non-disabled youth ( $n=49$, 23\%) (Howe et al., 1993). Youth with neurological disabilities had higher mean scores on the Child Behavior Checklist (CBC; both internalizing and externalizing symptoms), than those with non-neurological disabilities, who in turn had higher scores than the controls $(\mathrm{p}<.05)$ (Howe et al., 1993). While males had significantly higher scores on the overall CBC compared to females $(F=7.495, \mathrm{p}<.05)$, significant differences among females in all three groups were not observed (Howe et al., 1993). Further, after controlling for 
ethnicity and other demographic factors, significant differences were observed in regards to the total internalizing score of the CBC among males, with males with neurological disabilities having higher internalizing scores $(\mathrm{p}<.01)$ (Howe et al., 1993).

Another variation of the gradient can be observed in regards to sex and type of disability. In the study mentioned earlier in this chapter, involving 115 students with vision impairments (VI), 44 sighted students with disabilities/chronic conditions, and 607 non-disabled students, males with VI fared best on psychosocial well-being (Huurre \& Aro, 2000). Sighted males with chronic conditions reported the highest levels of distress symptoms $(M=9.7, S D=5.8)$, followed by non-disabled males $(M=7.0, S D=4.4)$; males with vision loss had the lowest levels of distress symptoms $(M=5.0, S D=3.9)(F$ $=10.69, \mathrm{p}=.001)($ Huurre \& Aro, 2000). The pattern was slightly different among females; females with chronic conditions reported the highest levels of distress symptoms $(M=12.6, S D=5.4)$, followed by females with vision impairments $(M=9.3, S D=6.3)$ and non-disabled females $(M=9.9, S D=5.3)(F=3.34, \mathrm{p}=.04)$ (Huurre \& Aro, 2000). Such differences by sex could be real, or influenced by different sample sizes, as there were a greater proportion of males with vision impairments compared to females with vision impairments, and conversely a greater representation of females in the nondisabled comparison groups.

In general, a type of "gradient" can be observed, with females reporting more psychosocial distress than males, and disabled females more than disabled males. However, findings from the empirical review are not consistent, likely due to differences 
in study designs and measures. Additional research is needed on the potential moderating role of sex in the association between psychosocial distress and disability.

\section{Peer Victimization and Psychosocial Distress: Youth with Disabilities}

This section will begins with review of what is known about the associations between peer victimization and psychosocial distress among youth with disabilities. However, to provide context, I start with a brief review of what is known about the associations between peer victimization and psychosocial distress generally. I then reviewed the literature informing the influence of exposure to peer victimization on youth with disabilities.

Researcher have found strong associations between peer victimization and psychosocial distress in the population overall. For example, in a meta-analysis conducted by Hawker and Boulton (2000), spanning 20 years, mean effect sizes for the association of peer victimization and depression in the general population was .29 among studies with no shared method variance $(\mathrm{p}<.0001)$, and .45 for studies with shared method variance $(\mathrm{p}<.0001)$. Symptoms and consequences of depression and other forms of psychosocial distress include suicide ideation and suicide attempts, which are also associated with peer victimization. A systematic review, involving 37 studies from 16 countries (of which 27 involved children and youth), also provides evidence to support bullying as a risk factor for increased suicidal ideation and suicide attempts (Kim \& Leventhal, 2008). Among general population studies, only three did not report an association between bullying and suicidal ideation. The authors reported increased odds 
of suicide ideation among victims ranging from 1.4 to 5.6, as well as increased odds of attempts of injury and suicide ranging from 1.5 to 5.4 (Kim \& Leventhal, 2008).

However, while there are several studies demonstrating separately the increased prevalence of psychosocial distress and peer victimization among YwD, few studies directly examined the association of peer victimization with psychosocial distress among youth with disabilities. These studies have primarily relied on correlational data. For example, several convenience studies, such as those conducted by Humphrey, Storch, and Geffken (2007) and Baumeister, Storch and Gefken (2008), sought to examine the association of disability status to peer victimization and psychosocial distress. Both of these studies consisted of samples largely white (more than $80 \%$ ), and both utilized the Child Behavior Checklist (CBCL Peer Victimization Scale), the Conner's Parent Rating Scale-Revised, the Children's Depression Inventory (CDI), and the Revised Children's Manifest Anxiety Scale (RCMAS). The study by Humphrey and colleagues involved 91 males and 25 females, ages 4 to $18($ mean age $=9.95, S D=3.5)$, diagnosed with ADHD between 1994 and 2003. Significant correlations were found between the CBCL peer score (proxy for peer victimization) and scores for anxious/ depressed $(.53, \mathrm{p}<.001)$, having social problems $(.80, \mathrm{p}<.001)$ and having psychosomatic symptoms $(.33, \mathrm{p}<.01)$ (Humphrey et al., 2007). The study by Baumeister and colleagues (2008) involved 68 males and 9 females, ages 4 to 18 (mean age $=11.30, S D=3.15$ ), diagnosed with learning disabilities and ADHD between 1994 and 2003. Significant correlations were found on the CBCL peer victimization score with being anxious/depressed $(.62, \mathrm{p}<$ $.001)$, and with having social problems $(.84, \mathrm{p}<.001)$ (Baumeister et al., 2008). 
Rarely, however, is the association of peer victimization and psychosocial distress examined among youth with disabilities in such a way as to determine "how" peer victimization impacts psychosocial distress among YwD, and "whom" among YwD are most exposed to peer victimization. Only three studies were identified which moved beyond bivariate correlations, two of which were retrospective cross-sectional studies using multivariate analyses. One of the two retrospective studies involved 276 adults with dysfluent disabilities recruited from the British Stammering Association (Hugh-Jones \& Smith, 1999). The mean age of participants was 38, with participants recalling experiences from 20 to 30 years earlier. Over seventy percent (71\%) reported being bullied at least once a week; most of which was reported during the ages of 11 to 18 (Hugh-Jones \& Smith, 1999). More than half of those bullied (63\%) reported short-term personal effects, such as increased anxiety and depression, and 32\% reported long-term personal effects (Hugh-Jones \& Smith, 1999). Participants were also asked if they found it hard to make friends, and if so, was “your stammer the only inhibiting factor?" (HughJones \& Smith, 1999, p. 144). Logistic regression produced two significant predictors in determining who would be more likely to be bullied: being male $(O R=2.67, \mathrm{p}<.02)$, and "always or usually had difficulty making friends" $(O R=6.26, \mathrm{p}<.001)$. Using multiple regression analysis, "difficulty making friends" was the only significant predictor in severity of bullying (Hugh-Jones \& Smith, 1999). Interesting enough difficulty in making friends due to one's stammering alone was not a significant predictor. 
The second retrospective study examined the effects of bullying on health among 1273 adults with intellectual disabilities, of whom $39 \%$ were between the ages of $16-25$ (Emerson, 2010). Over half of all respondents (56\%) reported being bullied in school (Emerson, 2010). After controlling for gender, age, level of support needs, and other demographic variables, those reporting being bullied in school were more likely to report feeling not happy sometimes $(34 \%, O R=1.5[1.15,2.01], \mathrm{p}<.01)$, sad "a lot" $(15 \%, O R$ $=1.9[1.24,2.93], \mathrm{p}<.01)$, and left out "a lot" $(13 \%, O R=2.3[1.46,3.64], \mathrm{p}<.001)$ (Emerson, 2010). Further, material resources (such as poverty, frequency of contacts with relatives and friends) significantly moderated the effects of exposure to disablism (rude and insulting behavior) and bullying in school on self-reported health status (Emerson, 2010). While both retrospective studies were limited with a reliance on memory and recall, they suggest that exposure to peer victimization affects the health of YwD with intellectual disabilities, and with stammering (dysfluent) disabilities.

In contrast to the preceding studies demonstrating simple associations between peer victimization and psychosocial distress for $\mathrm{YwD}$, results from one comparative study suggest that witnessing or being a victim of violence may be a more important predictor of suicide attempts than disability status alone (Blum et al., 2001). After controlling for age, gender, family situation, and race/ethnicity, students with mobility disabilities who reported witnessing violence (saw shooting/stabbing), or students who were victims of physical violence (threatened with gun or gun, jumped or stabbed), had the highest odds in reporting a suicide attempt $(O R=1.63, \mathrm{p}<.01)$, compared to other students with mobility disabilities who did not witness or experience violence (Blum et 
al., 2001). Students with learning disabilities who witnessed or experience violence were also more likely to report a past suicide attempt, compared to students with learning disabilities who did not witnessed or experienced violence $(O R=1.33, \mathrm{p}<.001)$. Similarly those with emotional disabilities who experienced or witnessed violence were more likely to report a past suicide attempt, compared to those with emotional disabilities who did not witness or experienced violence $(O R=1.3, \mathrm{p}<.001)$ (Blum et al., 2001). While victimization in this study included peer victimization, as well as victimization at home or by a stranger, the findings offer the first evidence to suggest that exposure to peer victimization could function as a potential mediator of the relationship between disability status and psychosocial distress.

Considering this body of research, there is a glaring lack of studies examining the extent to which the effect of disability status on psychosocial distress is mediated by exposure to peer victimization. It is rather remarkable that more research has not been done to investigate the potential association of peer victimization with psychosocial distress, taking into account disability status. Studies focused on psychosocial distress among YwD reviewed thus far did not attempt to explain the relationship between peer victimization and psychosocial distress among YwD.

One possible explanation for the relative lack of empirical studies examining the association between psychosocial distress and peer victimization may be a perception that peer victimization of YwD is a minor problem (Flynt \& Morton, 2008). Another possibility is that questions about exposure to peer victimization are not being asked directly. For example, the National Longitudinal Transition Study (NLTS2), which 
involved youth receiving special education services, did not include questions on peer victimization. Instead, the closest question that would indicate a degree of "interpersonal challenge" asked about difficulty in "getting along with other students" (Wagner, Newman, Cameto, Levine, \& Marder, 2007). Nearly a quarter of the 5222 (24.1\%) YwD enrolled in the NLTS2 study (Wave 2, 2003) reported difficulty in "getting along with other students" weekly or daily. At least $30 \%$ of the youth enrolled in the NLTS2 study in the following disability categories reported trouble "getting along with other students" at least weekly: multiple disabilities (33.9\%), hearing impairment (33.1\%), Autism (32.4\%), and intellectual disabilities (30\%). Nearly a quarter of students with learning disabilities $(21.5 \%)$ reported trouble "getting along with other students" at least weekly (Wagner et al., 2007). Wagner and colleagues (2007) reported that significantly more disabled youth (10.5\%), compared non-disabled youth (2.5\% of 2650 , age $15-19)$, reported difficulty in "getting along with other students" daily $(\mathrm{p}<.001)$; the matched comparison group was derived from the National Longitudinal Study of Adolescent Health (Udry, 1998). While caution is warranted in comparing the results from these two studies, their discrepant findings suggest the potential for disparity. Unfortunately, we do not know if interpersonal challenges in "getting along with other students" include experiences of peer victimization.

There is a scarcity in research examining the association of peer victimization and psychosocial distress among YwD. This is curious as a number of researchers have examined the mediating effects (as well as moderating) of peer victimization on the 
relationship of other identities (e.g., sexual orientation) and psychosocial distress. Below I briefly describe the findings which informed this dissertation research.

\section{Peer Victimization and Psychosocial Distress: Mediation and Moderation}

Likely in an effort to shift the gaze from the individual victim to the larger social context, a number of studies have tested hypothesized moderators and mediators of the relationship between peer victimization and psychosocial distress. In particular, in recent years, there has been an explosion of studies focused upon psychosocial distress and peer victimization, many of which involved LGBTQ youth who reported significantly higher levels of psychosocial distress and peer victimization compared to their heterosexual peers (Birkett, Espelage, \& Koenig, 2009; Espelage et al., 2008; Safren \& Heimberg, 1999; Young \& Sweeting, 2004).

Waldo and colleagues (1998) conducted one of the earliest studies, which involved testing hypothesized antecedents and consequences of peer victimization among LGB youth. Their study involved two cohorts of LGB youth LGB ages 15 to 21 . The first cohort, drawn from an earlier study by D’Augelli and Hershberger (1993), involved 192 LGB youth (mean age 18.9, 73\% male, 66\% white) recruited through community based centers serving LGB youth and young adults from 14 metropolitan centers in the U.S. The second cohort involved 54 undergraduates from a university in a rural area without access to nearby community centers serving LGB youth and young adults (mean age 20.2, 70\% male, 91\% white). Structural equation modeling did not support a direct pathway between peer victimization and psychosocial distress. Instead, low self-esteem was suggestive as a consequence of peer victimization (standardized parameter estimates 
$=-.16$ ), and as an antecedent of suicidality (standardized parameter estimates $=-.33$ ), and of depression/anxiety (standardized parameter estimates $=-.29$ ). In other words, peer victimization negatively affects self-esteem, which in turn produces greater psychosocial distress (Waldo et al., 1998).

Two retrospective studies provide support for peer victimization as a mediator of the relationship between gender non-conforming LGBT status and psychosocial distress. While both studies focused only on LGBT youth, they attempted to determine if the degree of visible non-conformity in gender expression was related to greater psychosocial distress. The first retrospective study conducted by Friedman and colleagues was based upon 96 gay, bisexual, and "other" (not heterosexual) males, with a mean age 20.3, who were recruited through gay community or university based organizations. Most of the participants were white (73\%). A path analysis found the relationship between "femininity" (measured by recall of gender non-conforming behaviors in childhood) and suicidality nonsignificant after the inclusion of exposure to peer victimization in the model, suggesting that exposure to peer victimization, not femininity, predicted greater psychosocial distress (Friedman et al., 2006) .

The second retrospective study, with a larger co-ed community sample of 245 LGBT youth from the San Francisco bay area (ages 21-25, $M=22.8 ; 51.4 \%$ Latino, $48.6 \%$ white, $46.5 \%$ male, $44.9 \%$ female, $8.6 \%$ transgender) found that LGBT-related peer victimization was a mediator of the relationship between gender non-conformity and depression $(\mathrm{p}<.01)$ using SEM analyses. Gender non-conformity was measured by a scale in which the young adults self-assessed the degree which they were "feminine" or 
masculine" during the age of 13 to 19 . The experience of peer victimization overall did not mediate the effect of gender non-conformity on depression, but rather the association between gender non-conformity as an adolescent and exposure to LGBT-specific victimization was significant $(r=.24, \mathrm{p}<.001)$, as well as the path from LGBT-specific victimization to current depression $(r=.39, \mathrm{p}<.001)$. Direct paths from gender nonconformity to depression were not significant, suggesting that the experience of prior LGBT-specific victimization between the ages of 13 to 19 , not gender nonconformity, predicted current depression (Toomey, Ryan, Diaz, Card, \& Russell, 2010).

Sex is thought by some to moderate the effect of peer victimization on psychosocial distress. In a study of 186 seventh graders in North Florida, ages 11 to 14 $(M=12.3)$, Dao and colleagues (2006) conducted hierarchical regression to determine the extent to which the effects of peer victimization on psychosocial distress was mediated by perceived risk of victimization. Once sex, exposure to peer victimization, and perceived risk of victimization were entered in the regression model, experience of victimization did not significantly predict psychosocial distress (Dao et al., 2006). Nabuzoka, Rønning, and Handegård (2009) found support for sex as a potential moderator of the relationship between peer victimization and psychosocial distress. Based upon a convenience sample of youth, ages 11-15 from four secondary schools in England (228 males, mean age $=12.4, S D=0.7 ; 287$ females, mean age $=12.7, S D=1.0)$, ANOVA tests revealed that female victims $(n=91$, mean score $=3.8)$ reported significantly more emotional symptoms, via the Strengths and Difficulties Questionnaire, than their male counterparts $(n=60$, mean score $=2.9)(\mathrm{p}<.05)($ Nabuzoka et al., 2009). 
Empirical studies of the general population suggest that sex may moderate the influence of peer victimization among youth with disabilities exposed to peer victimization, as males with disabilities tended to report better emotional health than females with disabilities (Huurre \& Aro, 2000; Miauton, et al., 2003; Surís, et al., 1996; Wolman, et al., 1994). However, to my knowledge, this influence has not been specifically tested.

\section{Section II Summary: Peer Victimization and Psychosocial Distress}

The aforementioned review examined research findings in regards to the prevalence and correlates of psychosocial distress and peer victimization among YwD. Clearly, there is increased prevalence of psychological distress among YwD, compared to their non-disabled peers, with distress generally highest among disabled females and lowest among non-disabled males. As aptly stated by Gesit, Grdisa, and Otley (2003), in discussing the increased risk of psychosocial "issues" among children and youth with chronic illnesses, the "literature is vast, but limited in its usefulness: criteria for the variables described, including chronicity and severity, are poorly defined; outcome measures are not standardized; and few randomized controlled clinical trials exist" (p.141). Similarly, the literature, while vast in charting psychosocial distress among YwD, is limited in explaining the "why" of psychosocial distress in the context of exposure to peer victimization.

Among those studies including peer victimization as a variable of interest, only three went beyond the typical analyses reporting on the correlation of disability status to either/both psychosocial distress and/or peer victimization (Blum, et al., 1991; Emerson, 
2010; Hugh-Jones \& Smith, 1999). None of these studies specifically examined whether exposure to peer victimization influenced greater psychosocial distress among youth with disabilities. In contrast, and consistent with the advice of Hawker and Boulton (2000), a relatively robust body of literature has formed to examine not only the nature and extent of peer victimization, but also the connections of such to psychosocial distress among other marginalized social groups. For example, in regards to LGBT youth and students, a number of researchers examined the extent to which sexual orientation status and exposure to peer victimization predict psychosocial distress (Birkett et al., 2009; Bontempo \& D’Augelli, 2002; Espelage et al., 2008; Friedman et al., 2006; Kosciw et al., 2010; Toomey et al., 2010; Waldo, Hesson-McInnis, \& D'Augelli, 1998).

Among the studies examining peer victimization among LGBT youth, results from several research studies suggest that sex could moderate the relationship between exposure to peer victimization and psychosocial distress, with more male bisexual and gay youth reporting psychosocial distress and peer victimization compared to their female lesbian/bisexual counterparts (Berlan et al., 2010; Kosciw et al., 2010). It is worth noting that similar patterns are seen among YwD, with both groups reporting more psychosocial distress than their non-disabled counterparts (Miauton et al., 2003; Wolman et al., 1994). However, in the case of $Y w D$, there is a difference in that the pattern appears to be driven first by sex and then by disability, with females reporting more psychosocial distress than males and disabled females more than non-disabled females (Miauton et al., 2003; Wolman et al., 1994). Unfortunately, none of the studies reviewed tested sex as a moderator on the relationship between peer victimization and psychosocial 
distress among $\mathrm{YwD}$, nor did they test peer victimization as a mediator between disability status and psychosocial distress.

\section{Conclusions and Interpretations}

Studies examining differences in peer victimization at the intersections of disability by sex, sexual orientation, and race/ethnicity are scarce, and thus little is known about which disabled youth are at heightened risk for psychological distress and peer victimization, or the role disability status plays in influencing exposure to peer victimization, alone or in combination with these other statuses. There is a lack of intersectional research, particularly in regards to YwD. The GLSEN report provided the most detail in regards to intersections among all of the other non-dominant culture identities (Kosciw et al., 2010). There is a need to expand beyond silo social categories, as intersecting social statuses appear to make a difference. Specifically there is a lack empirical research determining the extent to which disability status, in combination with sexual orientation and race/ethnicity statuses, and incorporating sex status, increases risk for peer victimization. As a consequence, disabled LGB youth, and disabled students of color experiencing peer victimization may be marginalized further by the tendency in research to look at one population at a time, thus making them "invisible" (Crenshaw, 1991).

In a meta-analysis published 11 years ago, examining the literature related to peer victimization and psychosocial distress, Hawker and Boulton (2000) concluded "there is little need now for further cross-sectional studies of peer victimization and psychosocial maladjustment. It is clear enough already that victims are distressed...it is time for 
victimization research to move on" (p. 453). While many researchers have moved on to explore issues of risk of exposure to peer victimization and the relationship to psychosocial distress, particularly among LGBT students, this is not true in regards to youth with disabilities. For example, the LGBT psychosocial research literature is distinguished by a focus on the association of exposure to a hostile social environment, in which "minority sexual orientation and gender atypicality are early magnets for maltreatment" (Cochran, 2001, p. 937). Consequently, industrious researchers conducted studies which suggest that after controlling for exposure to a hostile school climate, LGB students are no more likely to report depression compared to heterosexual students (Espelage et al., 2008). This is in contrast to the researcher's gaze on the problem of depression and suicidality among YwD, in which the dominant conceptualization of disability serve to keep the "gaze" on the disability or impairment as an inevitable causal factor in producing greater psychosocial distress among YwD.

The next chapter connects these empirical findings to theory in an effort to make sense of why YwD might be at higher risk for peer victimization, and why multiple nondominant culture identities may increase that risk further. I present a theoretical framework to explain why peer victimization might be more strongly associated with psychosocial distress than disability status per se. 


\section{CHAPTER III: THEORETICAL FRAMEWORK}

I first review theories explaining how and why subordinate social status(es), such as disability would predict greater exposure to peer victimization. I then discuss stress appraisal and coping response theories connecting peer victimization exposure to psychosocial distress, and conclude with presentation of the research questions, and discussion of how the research questions inform our empirical and theoretical knowledge.

To help guide the reader, a graphic representation of the relationship between the explanatory variables and the outcome variable(s) is presented in Figure 1; the shaded boxes in the roadmap represent key processes and variables related to this dissertation research.

\section{Antecedents of Peer Victimization}

Being different predicts different social experiences. The question is why peer victimization, and how? In this section, I describe several theories explaining societal and individual level responses to differences among individuals, and between groups.

\section{Stigma and Prejudice}

Stigma and prejudice theories are helpful in explaining the influence of dominant culture values and norms on individuals in society, in the form of stigmatization and prejudice. Allport (1954) with a focus on prejudice, and Goffman (1963) with a focus on stigma, both developed parallel theories to explain the ill effects of not being accepted as a member of a social group. Goffman referred to stigma as a socially constructed attribute that "is neither creditable or discreditable as a thing in itself" $(1963$, p.3). He focused on how the "other" managed stigmatization in interactions with other members 
of society. Another perspective, however, is the nature of group dynamics creating "us" and "them" group mentalities, justifying prejudice. The works of Allport, Goffman, and others led many scholars to conceptualize further the nature of stigma and prejudice, including Link and Phelan (2001) with their stigma conceptualization framework which bridged the micro-macro perspective of labeling (Campbell \& Deacon, 2006). Link and Phelan (2001) theorized labeling and stereotyping as leading to a separation of "us from them," which in turns leads to status loss and differential treatment or discrimination on the basis of the difference, supported by socio-cultural values and norms.

In a rather comprehensive review of 18 conceptual models involving stigma and prejudice, starting with the works of Allport and Goffman, the authors Phelan, Link, and Dovidio (2008) concluded that essentially, prejudice and stigma describe elements of the "same animal," with stigma representing broader processes that then support the production of prejudice. Phelan, Link and Dovidio proposed a typology organized around three functions of stigma, which in their words involved "keeping people down, keeping people in and keeping people away" (2008, p. 362). More about this typology is discussed below, as the concepts of stigma and prejudice are interwoven within mechanisms of oppression.

\section{Oppression, Stressors and Peer Victimization}

It is notable that the functions of stigma and prejudice proposed by Phelan, Link and Dovidio (2008) are reflected in works by structural oppression theorists such as Young (1990). Indeed, stigma and prejudice facilitate the mechanisms of oppression, which heightens one's risk of exposure to peer victimization. Young (1990) viewed 


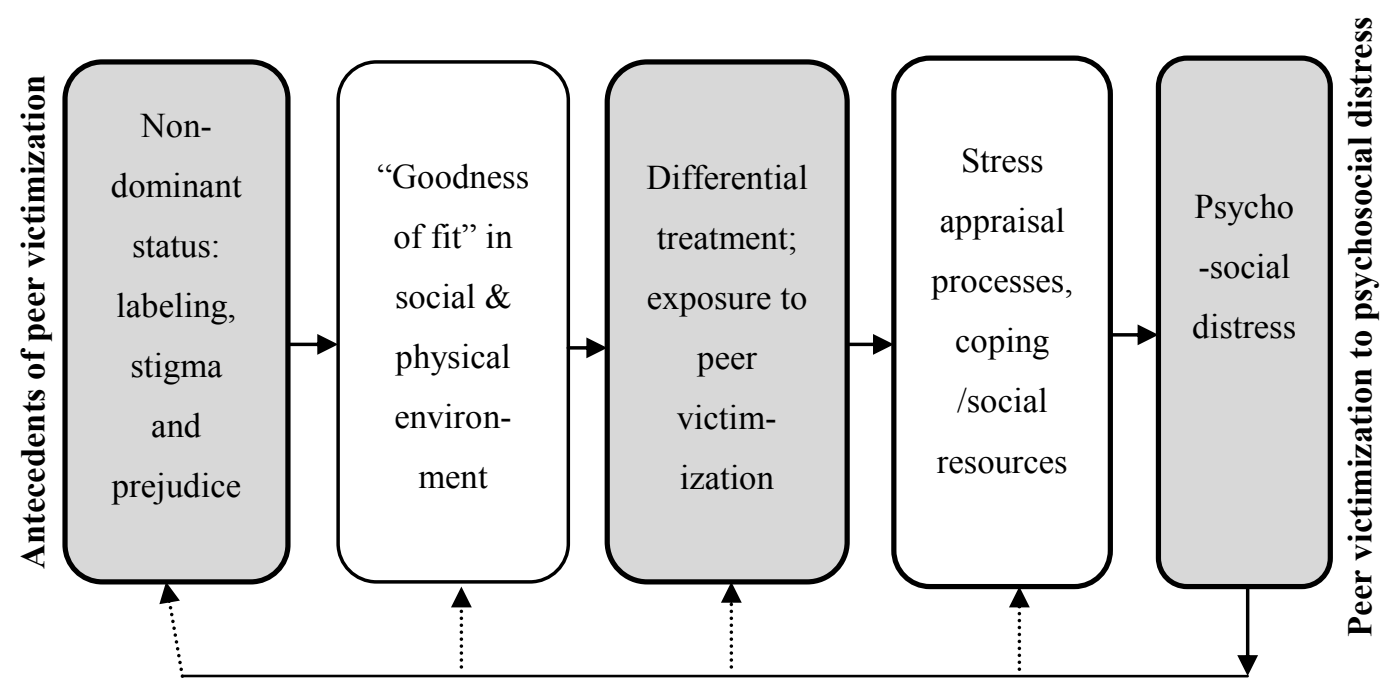

Figure 1. Theoretical road map 
oppression as a structural concept, as it refers to "systemic constraints on groups that are not necessarily the result of the intentions of a tyrant...Its causes are embedded in unquestioned norms, habits, and symbols, in the assumptions underlying institutional rules" (1990, p. 41). Young (1990) theorized that hegemonic adherence to dominant culture values and norms supports five forms of oppression, which include exploitation, marginalization, powerlessness, cultural imperialism, and violence, all of which serve to keep people “down," “in,” and/or "away.” As noted by Graham and Schiele (2010), conceptualizing oppression as taking one or more of these five forms of oppression suggested by Young (1990) provided space for multiple oppressions without the danger of assuming an "equality of oppressions" paradigm. In other words, the oppression experienced by LGBTQ youth may not involve the same form of exploitation experienced by females or people of color.

The typology proposed by Phelan, Link, and Dovidio (2008) is helpful in conceptualizing how and why YwD might experience more peer victimization compared to their non-disabled peers (Table 8). For example students are often kept "down" (and/or "out") in regards to equitable educational opportunities due to perception of their abilities and inabilities. As demonstrated in a qualitative study by Doubt and McColl (2003) youth with disabilities adopt a number of strategies to "fit" in high school settings, which can include "passing" or finding a "niche." An example of a "finding a niche" strategy is seen in the student who may be involved in school sports as a time keeper, "playing a position that no one else wanted to play" in order to be part of the school hockey team (Doubt \& McColl, 2003, p. 145). Students who are able to "pass" may choose to do so, but to 
LOST IN THE MARGINS

Table 8

Functions of Stigma and Prejudice in Context of Youth with Disabilities

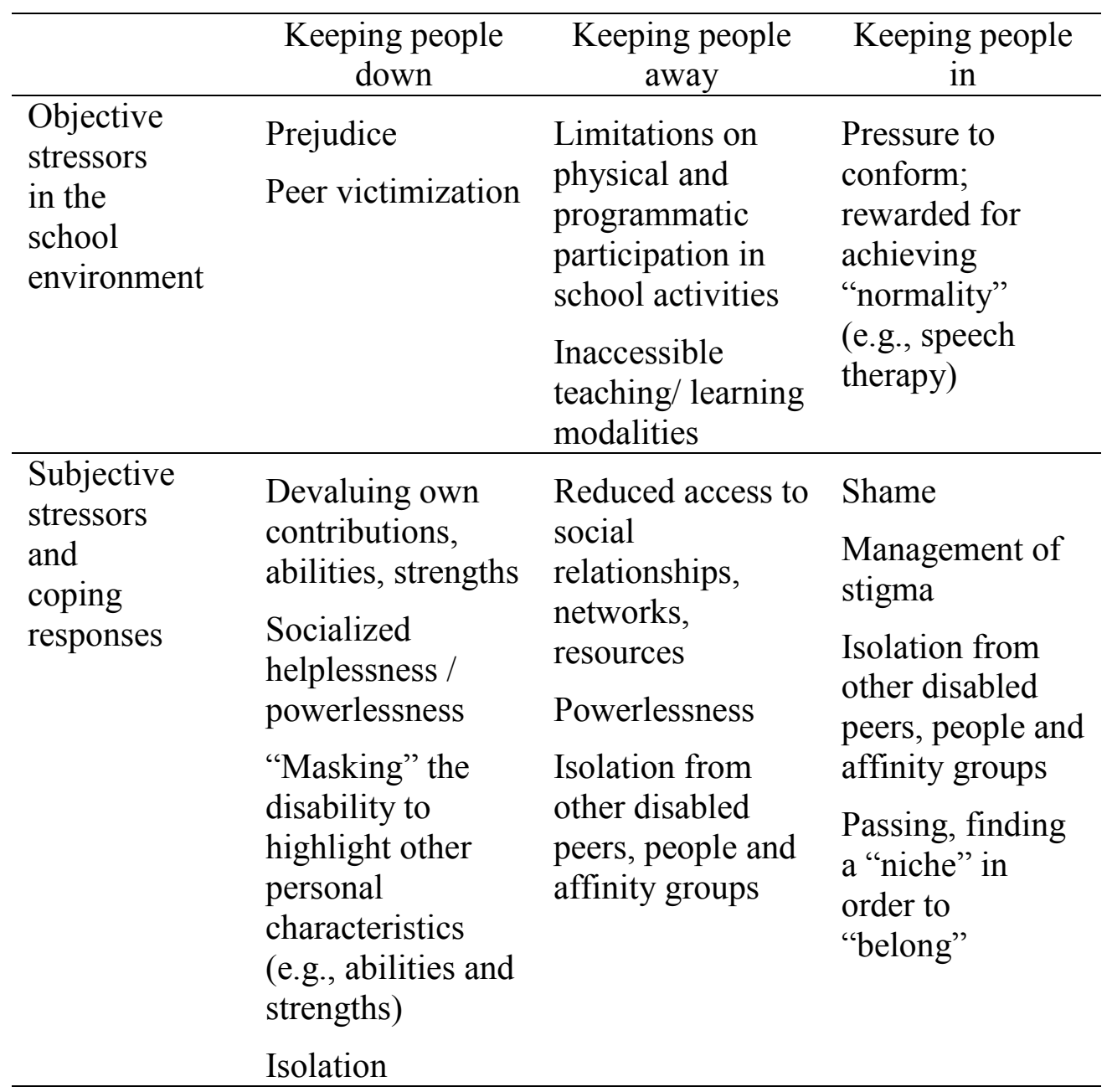

Note. Derived from typology developed by Phelan, Link, and Dovidio (2008). 
"pass," a student may feel pressure to forego asking for assistance, or participating. Both strategies have the effect of keeping YwD "out" of the mainstream.

\section{Non-Dominant Culture Status, Social Location, and Intersectionality}

What is the effect of having two or more stigmatized identities and does the effect change by place and time for each person? This section will begin with the concept of social location as it relates to non-dominant culture status, and then introduce the term "intersectionality" and the relevance of this conceptual lens to this study.

Social location refers "to the relative amount of privilege and oppression that individuals possess on the basis of specific identity constructs, such as race, ethnicity, social class, gender, sexual orientation, age, disability, and faith" (Hulko, 2009, p.48). The experience of one's social location is complex, fluid, and dynamic, changing with the social context (Hulko, 2009), meaning that the experience of the individual with one or more non-dominant culture (perceived) statuses is a function of time, place, and location. This is demonstrated in Mitchell's (2006) narrative, in which as a black deaf female she recalled how it "was uncanny because there was a drastic difference in the way my family recognized 'the deafness' and the way 'the deafness' was recognized by the world outside of my family, specifically by children and adults at school" (Mitchell, 2006, p. 138). The identity most salient varied by her social location at any given time.

Such fluidity of identity results in a gestalt-like social location being more than the sum of identities (Cole, 2009; Crenshaw, 1991); introducing complexity in understanding the experience of oppression based upon non-dominant identities. This complexity is reflected in Stuart's (1992) suggestion of simultaneous oppression. For 
example, the experience of being female, disabled, and identifying as a lesbian in high school, produces a social location that is more than the addition of disability status, sex, and sexual orientation statuses. The experiences and identity most salient to a disabled lesbian may differ when in the company with other LGB students, or with other disabled students who identify as heterosexual. Among other disabled students, she could be harassed due to her sexual orientation status. Among LGB students, she could be harassed due to her disability status. Clearly, exposure to peer victimization could vary by her social location, which is constantly shifting in time and place. Therefore, the degree of oppression that is experienced (as well as the degree of privilege) based upon one's social location is too complex to be characterized as "triple jeopardy" or "double jeopardy." Such characterization indicates an additive or multiplicative process (Hancock, 2007), implying “that social inequality increases with each additional stigmatized identity" (Bowleg, 2008, p. 314). These types of assumptions could bias research; for example, as risk factors related to peer victimization could be situated as a function of the number of stigmatized identities.

There is an important distinction between "social location" and "intersectionality." Crenshaw (1991), who first coined the term "intersectionality," conceptualized intersectionality as a way to elucidate and address structural oppressions and inequalities. This can be seen in a case study by Crenshaw which demonstrated that violence among women of color was interpreted and addressed differently by authorities and service providers than was violence among white women (Crenshaw, 1991). Crenshaw demonstrated how women who did not speak English were marginalized, as 
service providers did not take into account contextual differences based upon their social location. The "standards of support (provided by social service agencies) ignore the fact that different needs often demand different priorities in terms of resource allocation" (Crenshaw, 1991, p.1250).

Since Crenshaw's introduction of the term "intersectionality" (1991), debate within the academy has flourished, with the term "intersectionality" becoming a "buzzword" that has caused some confusion about the distinction between the effects of oppression based upon social location (Davis, 2008).However, there is growing consensus that the term "intersectionality" is best understood as a conceptual lens. Cole (2009) suggests that intersectionality represents a "paradigm for theory and research offering new ways of understanding the complex causality that characterizes social phenomena...” (p.179). Hulko (2009) also called for a distinction between intersectionality as an analytical lens or perspective, and social location, suggesting "intersectionality is best viewed as a metaphorical state of being, existing primarily in the consciousness of theorists, and that it should be no more than an analytical lens through which a researcher or theorist views the social world" (p. 48). An "intersectional" lens examining the prevalence of peer victimization and the effect on youth with more than one stigmatized identity, helps reveal the fluid nature of social location and the resulting "fit" with the social environment.

\section{Goodness-of-Fit with Social Environment, and Exposure to Peer Victimization}

In this section I examine how one's social status and social location affect one's fit with the social environment in school, and the subsequent exposure to stressors, such 
as peer victimization. In other words, I examine how "upstream" factors such as disability status affect one's vulnerability to being victimized by peers.

Social structures and social statuses, supported by dominant culture values and norms, configure educational, social and "health opportunities of social groups based on their placement within hierarchies of power, prestige and access to resources" (Commission on Social Determinants of Health, 2008, p. 26), or in other woods, the social location of the student within the school. As such, the high school social structures and physical environment influence the "goodness-of-fit" for people with non-dominant culture statuses. While non-dominant culture status can heighten exposure to stressors, the inaccessibility of the social and physical environment can negatively impact YwD (Simeonsson, Carlson, Huntington, McMillen, \& Brent, 2001), and the degree to which the "fit" in the school social environment is achieved (Doubt \& McColl, 2003).

The lack of "fit" in the environment in turn produces social vulnerabilities, which further weaken the "fit" in the social and physical environment. This can happen in several ways, such as limiting options for disabled youth to participate in extracurricular activities at school. Even if a school professes to be completely "ADA accessible", there are still limitations for some disabled students. For example, while there may be ASL interpreters available, not being able to directly communicate with one's peers is a limitation, and fosters social isolation. Social exclusion, produced by the labeling, marginalization and other mechanisms highlighted in Table 8, supports conditions for the "passive victim," a common type of victim cited in the peer victimization literature; with few/no friends, the passive victim is perceived to be different, and weak (in the case of 
boys), and is more vulnerable to exposure to peer victimization (Olweus, 1993; Orpinas $\&$ Horne, 2005).

Non-conformity with social norms heightens one's risk for peer victimization, with those doing the victimizing as enforcers of social norms (Juvonen \& Galván, 2009). Specifically, those perceived as "deviant" are targeted for punishment provided there is a power differential that enables the "bully" to carry out his/her task (Juvonen \& Galván, 2009). Peers doing the victimizing may single out not only the "passive victims," but also the "provocative victim," who is seen as being socially inappropriate or aggressive, thus inviting peer victimization. The cognitions of the "bully," per social-cognition theorists, may serve to justify peer victimization, such as distortion of consequences (it is not a big deal, it did not hurt), dehumanization (labeling), moral justification (e.g., being gay is sinful), and attribution of blame (e.g., the provocative victim) (Orpinas \& Horne, 2005). Many of these cognitions relate also to issues of non-conformity and stigmatization. The combination of a lack of fit, social vulnerability, and the power differential between the people involved in the peer victimization, is theorized to heightened exposure to peer victimization (Juvonen \& Galván, 2009; Olweus, 1993; Orpinas \& Horne, 2005).This section will review three types of peer victimization in the theoretical literature.

An examination of the type of victims commonly mentioned in the peer victimization literature provides support for the "non-conformity" thesis. Two common types of victims, frequently categorized, rather simplistically, as the provocative or aggressive victim, and the passive or submissive victim (Olweus, 1993; Orpinas \& Horne, 2005), suggest the failure of the victim to comply with dominant culture societal 
values and norms, which then increases exposure to peer victimization. Together, the ill fit and resulting social vulnerabilities heightens one's exposure to objective stressors, including peer victimization. A third type of victim, elucidated by Orpinas \& Horne (2005) is the relational victim, which may not be related to non-conformity in the social environment, or to a non-dominant culture status (relative to the individual doing the peer victimization). The relational victim is typically one who threatens the social position, power, or relationships of the person doing the victimizing.

\section{Connecting Peer Victimization to Psychosocial Distress}

In the preceding sections I provided several plausible explanations for how being different could result in greater exposure to stressors such as peer victimization (see Figure 1). In this section, I review theoretical models of stress and coping response explaining differential psychosocial adjustment among $\mathrm{YwD}$, particularly within the context of peer victimization.

As seen in Chapter Two, the social context influences psychosocial health, as well as exposure to peer victimization. Excluding those with emotional and mental health disabilities, the disability in the body by itself does not automatically cause higher psychological distress. Indeed, as Wallander, Thompson, and Alriksson-Schmidt (2003) concluded in their overview of psychosocial adjustment of children with physical conditions, "a simple or direct universal relationship between chronic physical conditions and psychosocial adjustment does not exist. Rather a wide range of responses to the source of life stress is evidenced" (2003, p. 144). 
There are many variations of the "stress model" explaining the connections between stressors and health outcomes, ranging from a focus on the biological effects of stress on well-being, to the social determinants of health model which attempts to incorporate social structures as well as materials, psychosocial and behavioral pathways (Brunner \& Marmot, 2006; Commission on Social Determinants of Health, 2008; Lazarus \& Folkman, 1984). As this dissertation research focuses specifically on the psychosocial health of YwD in the context of exposure to peer victimization, biological and larger social determinants models will not be reviewed.

\section{Social Stress Theories}

Social stress theories in the sociological tradition are those in which social structures and social statuses produce both stressors and psychosocial health outcomes, with coping resources situated as a moderator of the relationship between exposure and psychosocial health outcomes (Lazarus \& Folkman, 1984; Meyer, 2003; Meyer, Schwartz, \& Frost, 2008). Stress and response models, with stressors explaining increased psychosocial distress, have their roots in the seminal theoretical work by Lazarus and Folkman (1984) on stress appraisal and coping. Lazarus and Folkman (1984) defined psychological stress as "a particular relationship between the person and the environment that is appraised by the person as taxing or exceeding his or her resources and endangering his or her well-being" (Lazarus \& Folkman, 1984, p. 19). While the authors acknowledged the effects of "objective" stressors, such as major events, or peer victimization, they did not incorporate the impact of these stressors in their stress appraisal and coping model; they were much more interested in the response to objective 
stressors, than in the nature of the objective stressors themselves. Lazarus and Folkman (1984) suggested that cognitive appraisal and coping processes mediated psychological outcomes. However, as pointed out by Holmbeck (1997), the manner in which they discussed these processes suggested moderation processes, not mediation processes. Variants of Lazarus and Folkman's stress model have been produced over the years, including several coping and stress models specific to children with disabilities, such as the Thompson's Stress and Coping model (Thompson \& Gustafson, 1996), and the Disability-Stress-Coping Model of Adjustment developed by Wallander and Varni (1995). However, both fail to explicate the ill effects of stigma and prejudice, with the child's disability positioned as the stressor (Thompson \& Gustafson, 1996; Wallander \& Varni, 1995). The "minority stress theory," on the other hand, provides a model that applies well to youth with disabilities (Meyer, 2003; Meyer et al., 2008).

\section{Minority Stress Theory}

Minority stress theory as explicated by Meyer (2003), in the context of sexual orientation status, goes further to illustrate how one's non-dominant culture identity moderates the relationship between non-dominant culture status and subjective stress processes, as well as access to and utilization of coping and social supports. This model acknowledges the complexity of identity, with identity as an antecedent of potential exposures to stressors, and the role of identity either as a risk or resilience factor in responding to stressors. Below I review the model in depth, showing how the model is relevant to those occupying a disability status. 
Environment, non-dominant culture status, and identity as antecedents. The student's social location, interaction with the environment, non-dominant culture status and identity all relate to the earlier discussion on the "goodness-of-fit" in the social environment. Again, referring to the phenomenological study involving seven high school students with physical disabilities, Doubt and McColl (2003) identified a number of conditions indicating a less than desirable fit with the social environment, such as exclusionary peer reactions and inaccessible extra-curricular activities. Limited accessibility in the environment impacts the degree to which students can be independent (Fange, Iwarsson, \& Persson, 2002), participate in non-academic offerings in school, such as band, chorus, student paper, and student government activities (Simeonsson et al., 2001), as well as sports (Doubt \& McColl, 2003). Classroom setting (e.g., placement of student in the front of the class) and teacher style and approach to the disabled student (e.g., managing peer relations of the youth with a disability) (Baker \& Donelly, 2001) can also directly impact the "goodness-of-fit" of the student with a disability in school settings, thereby introducing objective forms of stressors into the life of YwD.

\section{Minority stress processes in response to stressors. Stigma management and} acceptance of one's non-dominant culture status, whether it is disability or LGBTQ status for example, has the potential of being a risk factor or protective factor when faced with objective stressors. As seen in Chapter Two, perceived subjective stressors relating to one's non-dominant culture status and/or identity are more likely to be found to moderate the impact of one's non-dominant culture status on one's psychosocial health. 
The internalized stigma resulting from labeling and stereotyping in dominant culture provides much of the basis for the processing of subjective stressors. Strategies facilitating school integration, such as those highlighted in Doubt and McColl's study (2003), contain hints of internalized stigma. For example, "masking" as a strategy to draw attention away from one's disability (which may also be a form of "passing") could suggest internalized stigma. "Finding a niche," which was mentioned earlier, is another strategy for integration at school, with the student choosing to participate in a role not desired by non-disabled peers (Doubt \& McColl, 2003).

In order to take advantage of school-based or community-based supports, one must first perceive that such supports are available. However, internal stressors relating to one's non-dominant culture status and/or identity moderates perceived availability and use of coping and social resources. For example, a disabled student praised for not "needing" any "special accommodations," could find it difficult to seek out such services when needed. In this way, one's own self-esteem as a disabled person moderates use of resources; those with greater self-esteem may access and use more services, while those with less self-esteem may access resources less often.

Research focusing on the intersections of disability, race/ethnicity and gender reveal a number of appraisal and coping strategies, varying by social location in response to objective and subjective stressors (McDonald, Keys, \& Balcazar, 2007; Mitchell, 2006; O'Toole, 2000; Petersen, 2006; Petersen \& Gallagher, 2006). Indeed, McDonald, Keys and Balcazar (2007), based upon their qualitative research with a small group of college students with learning disabilities, highlighted several coping responses, such as 
removing oneself from the environment, and rejecting negative messages based on dominant cultural values.

The minority stress model is somewhat iterative, with the recognition that experienced stressors impact psychosocial distress, which then can contribute to a worsening of the goodness-of-fit with one's environment. A qualitative study focused upon understanding the meaning of participation for people with disabilities identified self-esteem as a prerequisite for full participation, and yet, lack of access makes full participation challenging (Hammel et al., 2008). Which comes first- high self-esteem or access? Similarly, non-dominant culture status shapes one's social experience through the "goodness-of-fit" of the environment and exposure to stressors, such as peer victimization. Furthermore, stigma management and acceptance of one's non-dominant culture status influences the relationship between exposure to stressors, and psychosocial health outcomes. A disabled person "passing” may find an improved goodness-of-fit with the environment, thereby reducing exposure to potential peer victimization; however, “passing” may make it difficult to hold pride in one's self. Further coping responses influences the likelihood of exposure to future stressors. For example, a person who is highly depressed may become socially isolated and withdrawn. The resulting social isolation can negatively affect the goodness-of-fit, and thus heighten one's vulnerability to exposure to peer victimization.

\section{Summary and Implications}

The relevance of the social location of students with disabilities in high schools to exposure to peer victimization lies within the variations among youth with disabilities 
with respect to sex, sexual orientation, and race/ethnicity. If we understand the degree to which there are variations in the nature and extent of the social problem of interest, based on multiple social identities and statuses, we will be more effective in designing interventions and policies to address the social problem. This may seem obvious, but the continual focus on one social category or another, without regard to differences between or within groups hinders efforts of social workers and others to understand and design appropriate and accessible interventions. This is the danger of binary type practices that look at, for example, disabled students, or LGBT youth as separate entities, which further marginalizes students who are both disabled and gay (Crenshaw, 1991). As stated by Cole (2009) "focusing on a single dimension in the service of parsimony is a kind of fake economy" (p.179). Crenshaw illustrated this danger eloquently; "Because of their intersectional identity as both women and people of color within discourses that are shaped to respond to one or the other, the interests and experiences of women of color are frequently marginalized within both" (Crenshaw, 1991, pp. 1242-1243). Another danger is the "equality of oppression" paradigm coined by Graham and Schiele (2010), in which the effects of oppression experienced by people with disabilities, competes with the effects of oppression experienced by people of color, resulting in a type of "Oppression Olympics" (Hancock, 2007).

The implications of utilizing an intersectional perspective in research examining the relationship between exposure to peer victimization and psychosocial distress is in the idea that one non-dominant culture identity (e.g., disability), among many that an individual may hold, should not be assumed as the primary determinant of exposure to 
objective stressors such as violence (Daley, Solomon, Newman, \& Mishna, 2007). Nor should one's subjective stress appraisal and coping strategies be assumed to be based upon one non-dominant culture status (Daley et al., 2007). Synthesis of the explanatory theories and theoretical frameworks in this chapter suggests that the minority stress theory provides a plausible explanation for how objective stressors, such as exposure to peer victimization, result in psychosocial distress. There is empirical support for the theoretical underpinnings outlined in this chapter, such as the relationship between disability status and participation in school, negatively affecting the goodness-of-fit with the school environment (Doubt \& McColl, 2003; Simeonsson et al., 2001). This research applies an intersectional approach, with critical thought to what makes sense in thinking of social categories, and how doing so serves in working across and beyond differences (Nash, 2008). Specifically, in the context of peer victimization experienced by high school youth, rather than examining who is at most risk for peer victimization by a single social status, this dissertation research examines who is at most risk across multiple social statuses in such a way that avoids the problem of the "Oppression Olympics" (Hancock, 2007).

\section{Research Questions and Hypotheses}

Directly informed by the preceding research findings and theoretical frameworks, this dissertation study addresses the research questions associated with the following three aims. 


\section{Research Questions and Hypotheses: Aim \#1}

The first aim, "To examine the extent to which disability status is associated with exposure to peer victimization" is associated with one research question and one hypothesis.

Research question 1.1. Is disability status associated with peer victimization, and if so, to what extent?

Hypothesis 1.1.1. Students with disabilities would be significantly more likely to report peer victimization compared to non-disabled students.

\section{Research Questions and Hypotheses: Aim \#2}

The second aim is as follows: Taking an intersectional approach, investigate the extent to which disability status in combination with two sets of identity variables (sexual orientation, sex; and racelethnicity, sex) is associated with exposure to peer victimization. There are three research questions associated with this aim, described below along with their associated hypotheses.

Research question 2.1. Taking an intersectional approach, what is the relationship between (a) disability status with each of the three demographic variables sex, sexual orientation, and race/ethnicity, and (b) peer victimization, controlling for other demographic variables?

This research question involves three sets of analyses to determine the extent to which disability status in combination with one of three other variables (sex, race/ethnicity, and sexual orientation) is associated with exposure to peer victimization. 
Hypotheses 2.1.1. Disability and sex. I hypothesized that disability status would be the strongest predictor of exposure to peer victimization, with disabled students, compared to non-disabled students, more likely to report peer victimization, regardless of sex. Further, I hypothesized that greatest exposure to peer victimization would be among females with disabilities, while the least exposure to peer victimization would be among non-disabled males.

Hypotheses 2.1.2. Disability and race/ethnicity. In examining the intersections of disability of race, the overall hypothesis was that students with disabilities of any race/ethnicity would be more likely to report peer victimization, compared to students without disabilities of any race/ethnicity. However, I also hypothesized that students of color and/or Latino would be represented at both ends of the spectrum, with non-disabled students of color and/or Latino students least likely, and disabled students of color and/or Latino most likely, to report peer victimization. Further, white, non-Latino students with disabilities would be more likely to report peer victimization, compared to white, nonLatino students without disabilities. In this hypothesis, disability status is expected to be the strongest predictor of exposure to peer victimization, even when combined with race/ethnicity.

Hypotheses 2.1.3. Disability and sexual orientation. I hypothesized that the greatest exposure to peer victimization reported would be among LGBQ students with disabilities, and that non-disabled males identifying as heterosexual and not having same sex experience as a group would report the lowest rates of exposure to peer victimization. 
In this hypothesis, sexual orientation was expected to be the strongest predictor of exposure to peer victimization, even when combined with disability status.

Research question 2.2. Taking an intersectional approach, what is the relationship between (a) disability status with sex, and racelethnicity, and (b) peer victimization, controlling for other demographic variables?

Hypotheses 2.2.1. Disability, sex, race/ethnicity and peer victimization. As in 2.1.2, I hypothesized that students with disabilities of any race/ethnicity compared to white, non-Latino students with and without disabilities, would be more likely to report peer victimization, regardless of sex. Specifically, I hypothesized that male non-disabled students of color and/or Latino would be least likely, and female students of color and/or Latino with disabilities most likely to report exposure to peer victimization. To summarize these hypotheses, disability status was expected to be the strongest predictor of exposure to peer victimization, even when combined with sex and race/ethnicity.

Research question 2.3. Taking an intersectional approach, what is the relationship between (a) disability status with sex, and sexual orientation, and (b) peer victimization, controlling for other demographic variables?

\section{Hypotheses 2.3.1. Disability, sex, sex orientation and peer victimization. I}

hypothesized that male GBQ students with disabilities would be most likely, and male students without disabilities who identify as heterosexual and did not report having same sex experience would be least likely, to report peer victimization. I did not expect that disability status will be the strongest "predictor" of being exposed to peer victimization, after considering sex and sexual orientation status. Further, I anticipated that being male 
would be "protective" for students identifying as heterosexual, but not for GBQ males, whom I hypothesized would be most likely to report peer victimization compared to males and females identifying as heterosexual, with or without disabilities. To summarize these hypotheses, sexual orientation was expected to be the strongest predictor of exposure to peer victimization, even when combined with sex and disability status.

\section{Research Questions and Hypotheses: Aim \#3}

Three research questions were associated with the third aim: "To determine if disability status is associated with psychosocial distress, and if so, determine if the effects of disability status on psychosocial distress are mediated by exposure to peer victimization."

Research question 3.1. Is disability status associated with psychosocial distress, and if so, to what extent?

Hypotheses 3.1.1. Disability and psychosocial distress. I hypothesized that disabled students, compared to non-disabled students, would be more likely to report symptoms of psychosocial distress.

Research question 3.2. Is the relationship between disability status and psychosocial distress mediated by exposure to peer victimization?

\section{Hypotheses 3.2.1. Mediation: disability, psychosocial distress, and peer}

victimization. I hypothesized that exposure to peer victimization would partially mediate the relationship between disability status and psychosocial distress. Full mediation was not hypothesized simply due to the omission of other contextual factors that are 
independently associated with psychosocial distress (e.g., recent death in the family, failing grades, alcohol and drug use).

Research question 3.3. Is the mediated effect of peer victimization on the relationship between disability status and psychosocial distress moderated by sex?

\section{Hypotheses 3.3.1. Moderated mediation: sex, psychosocial distress, and peer}

victimization. I hypothesized that that the degree to which the mediation effects of peer victimization on psychosocial distress would differs by sex; specifically, the medication effect peer victimization would be stronger for females compared to males.

\section{Conclusion}

The empirical literature and theoretical review informed the development of the research questions and hypotheses in this dissertation research. We do not know the degree to which disability status, alone and in conjunction with other identity statuses, produces differential levels of exposure to social oppression. It is plausible that disabled students of color and disabled LGB students both experience greater exposure to peer victimization compared to non-disabled students and non-disabled LGB students. It is also possible that disability status is "protective" when combined with one or more of these stigmatized identities. This may be due to a paternalistic notion in which the youth with a disability is considered "hands off" if the bully is focused upon targeting LGBT students or Latino students, for example. Or it may be due to "intersectional invisibility" of two or more stigmatized identities, which makes the disabled gay student, for example, “invisible" as a target for bullying (Purdie-Vaughns \& Eibach, 2008). The findings from this research clarify the answers to some of these important questions. 
This dissertation research makes an important contribution to the existing literature, as it strengthens our understanding of who is most likely to experience peer victimization, which is important information for those involved in preventing and reducing peer victimization in schools. Further, the results of this study encourage future research in connecting disability status to objective and subjective stressors based upon environmental and structural biases. Therefore, it is imperative that in this context, researchers consider disability status in relation to other social identities.

Reflecting upon the ways in which disability status has been contextualized in the empirical research and in the review of explanatory and theoretical models, brings to mind the three waves of scholarship in regards to racial prejudice proposed by Dovidio (Dovidio, 2001). Scholars and researchers in the first wave may have assumed that racial prejudice reflected societal pathology, particularly in the context of overt racism up to the 1960s. The second wave of research acknowledged and tested the existence of aversive racism (unconscious), and the third wave connected underlying implicit and explicit attitudes to different forms of prejudicial behaviors. Perhaps, in regards to disability attitudinal and prejudicial scholarship, we have our own "waves." It is time for researchers to move into the third wave, and examine the relationship between underlying implicit and explicit attitudes experienced by youth with disabilities and the production of objective stressors, such as peer victimization. To do this it is important to conceptualize disability status as a marker of difference, which in turn increases exposure to peer victimization. 


\section{CHAPTER IV: METHODOLOGY \\ Study Design}

I conducted secondary analyses of data collected in the 2008 Oregon Healthy Teen (OHT) survey in order to answer the research questions presented in Chapter Three. The OHT survey was derived from the Youth Risk Behavior Survey (YRBS), a biennial national survey, developed by the Centers for Disease Control and Prevention (CDC) (Brener et al., 2004). The YRBS was designed to collect data about the prevalence of six categories of health behaviors that are related to leading causes of morbidity and mortality among teens (Brener et al., 2004; Centers for Disease Control and Prevention, 2005). In reliability studies conducted on the national YRBS dataset, items that were found to be significantly associated with a different response in a two-week re-test were dropped or revised (Brener et al., 2004). The 2008 Oregon Healthy Teen Survey, which was administered to $8^{\text {th }}$ and $11^{\text {th }}$ graders, included questions about disability, as well as sex, race/ethnicity and sexual orientation. These demographic questions, along with measures of peer victimization and psychosocial distress, made the 2008 OHT data set suitable for this study.

\section{Sampling Methodology}

OHT sampling. The 2008 Oregon Healthy Teen survey (OHT) design utilized a probability design. The state was divided into eight regions, and School Districts (SD) were sampled from within regions to ensure representation of all regions on the survey. Certain large high schools within school districts were also randomly sampled from within the SDs. The randomization process used in the OHT is intended to minimize 
possible selection biases, and minimize sampling error with stratification of school regions (Babbie, 1990; Heeringa, West, \& Berglund, 2010a). There was no "replacement" for those schools and students who chose to opt out of the survey. However, the Oregon Health Authority (OHA) provided the option for all school districts to benefit from participating in the Oregon Healthy Teen survey (OHT), even if they were not part of the randomized sample, as well as the option to opt out. Among the school districts that consented to be part of the survey, schools were randomly selected to be part of the weighted sample.

Within each school, all $8^{\text {th }}$ and $11^{\text {th }}$ graders were given the opportunity to participate in the survey during one school period, typically a class period that all students take, such as English or physical education (R. Boyd, personal communication, April 11, 2011). Survey administrators used passive consent protocols in combination with an active notification process. Schools notified parents of randomly selected students about the upcoming survey, and gave parents an opportunity to refuse consent. During the time of administration of the survey, school officials also gave students the option to decline participation, or to skip questions that they did not want to answer. School officials followed standardized procedures in the administration of the survey to protect student privacy and facilitate anonymous participation, with classroom teachers trained in advance on survey protocols. It took about 30 minutes for students to complete the survey. Personal identifiers were not included in student's questionnaire booklet; booklets from the students, upon completion were placed in an envelope. The last student completing the survey was asked to seal the envelope for the booklets (surveys) 
completed in his/her classroom. The OHT survey was also available in Spanish. Of the more than 28,000 students who participated in the 2008 OHT survey, the randomized weighted sample of $11^{\text {th }}$ graders consisted of 7091 students from 86 schools, representing a student response rate of $65.6 \%$, and a school response rate of $75.8 \%$ (R. Boyd, personal communication, April 11, 2011).

\section{Data Management}

Analysis of the OHT data set, with a single stage cluster design (in which school districts were randomly sampled from within each of eight regions in the state), required the use of statistical software capable of handling complex survey data. I used Stata statistical software (version 12), which is capable of such, for all data management processes and statistical analyses (Centers for Disease Control and Prevention, 2005; StataCorp, 2011). I obtained a data sharing agreement from the OHA giving me permission to use the data set, which did not contain any identifying information (Appendix B \& C). Recoding steps used to facilitate analysis with Stata are detailed below.

\section{Construction of the Data Set for Analyses}

If analyses are not conducted adjusting for a complex survey design, biased standard errors make it more likely that the estimate is determined to be statistically significant (rejecting the null hypothesis) when it is not; a Type 1 error (Tabachnick \& Fidell, 2007). Due to the cluster sampling design (by school districts, and in some cases, large schools), there is greater likelihood of homogeneity which can lead to underestimated standard errors (Thomas \& Heck, 2001). This is compounded by the 
unequal probabilities of selection since certain school districts/schools may have been oversampled. The design effects from these two elements of the complex survey design can be decreased by stratification (regions), which is often done to increase the precision of the sample (Heeringa et al., 2010a). Therefore, in order to adjust for the complex survey design of the OHT survey, I needed to account for the stratification by regions, sampling of school districts, and weighting of observations. Below I describe more about the design of the survey as it pertains to adjusting for the complex survey design (For more more information see: Korn \& Graubard, 1991). Then I describe specifically what was done in the case of the OHT dataset.

First, in regards to sampling, there were a total of 86 schools from 76 school districts. Since the survey design involved random sampling of school districts from eight regions, the primary sampling unit (PSU) is represented by 76 school districts. The regions represent the stratum, and the number of PSUs (school districts) within each stratum ranged from seven to twenty.

The weighting of observations is another feature of many complex survey data sets (Korn \& Graubard, 1991). In the case of the OHT dataset, the OHA staff created a variable representing a weight value for each participant. The weighted value represents the probability of students within a school of being selected (Babbie, 1990), thus students within the same school had the same weighted value.

Normally, in the process of preparing the complex survey dataset for analysis, the PSU (school districts) and strata (regions), in addition to a weighted variable, are known to the researcher. However, since the dataset received from the OHA was set up to 
protect the identities of students, schools, school districts and regions, I was not able to identify the school or school district associated with a record. This made it difficult to “declare survey design” in Stata, a necessary prerequisite for all analyses. Fortunately I was able to rely on syntax produced by OHA staff which allowed me to input the required identifiers for the school districts and regions in a way that preserved their anonymity. This made it possible for me to "declare survey design" in Stata, specifying the regions as stratum identifiers and the school districts as the primary sampling units. The syntax received from OHA staff also created a variable that represented the number of total districts within each region, which was designated as the finite-population correction (FPC) variable; the FPC reduces the sampling variance when sampling is done without replacement (Heeringa, West, \& Berglund, 2010b; StataCorp, 2007), as in this case. In converting the dataset from SPSS to Stata, care was taken to retain the original missing values which distinguished the reasons for missing by the respondent (e.g., skipped, marked as missing due to inconsistent or implausible response).

\section{Dependent and Mediator Variables}

Peer victimization. One harassment question from the survey was used as a measure of peer victimization. The question, asking about harassment experienced in the past 30 days, was preceded by this explanation to students: "Harassment can include threatening, bullying, name calling or obscenities, offensive notes or graffiti, unwanted touching, and physical attacks" (see Table 9) (Oregon Department of Human Services, 2008, p. 5). Students were able to choose up to six reasons for the harassment they experienced, such as due to race, sexual attention/comments, LGB status, other physical 
characteristics, group of friends, or "other" reasons. Students reporting experience of harassment in the past 30 days due to any of the six reasons were categorized as reporting peer victimization, resulting in a dichotomous measure of peer victimization. Participants not answering the question about peer victimization were excluded from all bivariate and multivariate analyses $(n=373)$.

Psychosocial distress. Psychosocial distress was measured by five OHT survey items, which make up a five item Mental Health Inventory (MHI-5). The MHI-5 is a validated screening tool for depression and anxiety among adults (Cuijpers, Smits, Donker, ten Have, \& de Graaf, 2009; Rumpf, Meyer, Hapke, \& John, 2001), and students as young as 16 (Strand, Dalgard, Tambs, \& Rognerud, 2003; Yamazaki, Fukuhara, \& Green, 2005). All five questions have been shown to work together well as a scale (Rumpf et al., 2001; Strand et al., 2003; Yamazaki et al., 2005); MHI-5 Cronbach alphas across studies range from .83 to .91 (Veit \& Ware, 2008).

For each MHI items on the OHT survey, students were asked how much of the time in the past 30 days they "been a very nervous person," "felt downhearted and blue," "felt so down in the dumps that nothing could cheer you up," "felt calm and peaceful," and "[had] been a happy person," on a scale of 1 ("None of the time") to 6 ("All of the time"). The positively worded statements (happy, calm) were reverse scored for the purposes of calculating the mean and the MHI-5 score. Cronbach's alpha was calculated to be 0.85 .

A mean MHI-5 score was calculated for each student, adjusting for the number of items to which a student responded, only if the student answered at least four of the five 
MHI-5 items as recommended by Ware and colleagues (2007). The final MHI-5 score was derived by linearly transforming the MHI-5 score for each participant to a $0-100$ scale as recommended by Ware and colleagues (2007).

Transformed score $=100 \cdot[($ mean MHI-5 score - lowest score possible $) /($ score range)*]

*Score range $=$ highest possible score - lowest possible score

Following the calculation of an adjusted mean MHI-5 score, the rate of missing for a MHI-5 total score, based upon the mean score, was just under $4 \%(3.52 \%, n=269)$. Participants not answering at least four of the five MHI items were excluded from mediation analyses.

\section{Independent Variables}

An indicator variable for each status variable (disability, race/ethnicity, sex, sexual orientation) was created to facilitate analyses (Heeringa et al., 2010a). For example, I assigned " 1 " to a case if disabled, and " 0 " if not. I describe below the rationale and process used for recoding variables for this study. Table 10 provides the counts and weighted means of the breakdowns of all independent variables described below. 
Table 9

Description of Relevant Study Variables

\begin{tabular}{|c|c|}
\hline Variable Description & Coding \\
\hline \multicolumn{2}{|l|}{ Dependent and Mediator Variables } \\
\hline \multicolumn{2}{|l|}{ Peer Victimization $^{\mathrm{a}}$} \\
\hline $\begin{array}{l}\text { During the past } 30 \text { days, have you ever been harassed at school (or on } \\
\text { the way to or from school) in relation to any of the following issues? }\end{array}$ & $\begin{array}{l}0 \text { if } \mathrm{G} ; 1 \text { if } \mathrm{A}, \mathrm{B}, \mathrm{C}, \\
\mathrm{D}, \mathrm{E} \text {, or } \mathrm{F} .\end{array}$ \\
\hline \multicolumn{2}{|l|}{ Psychosocial Distress [Mental Health Inventory (MHI)- 5 items] } \\
\hline \multicolumn{2}{|l|}{ During the past 30 days, how much of the time have you: ${ }^{c}$} \\
\hline Been a very nervous person? & \multirow{5}{*}{$\begin{array}{c}\text { Scale 1-6: } \\
1 \text { if } \mathrm{A} ; 2 \text { if } \mathrm{B} ; 3 \text { if } \mathrm{C} ; \\
4 \text { if } \mathrm{D} ; \\
5 \text { if } \mathrm{E} ; 6 \text { if } \mathrm{F}\end{array}$} \\
\hline Felt calm and peaceful? & \\
\hline Felt downhearted and blue? & \\
\hline Been a happy person & \\
\hline Felt so down in the dumps that nothing could cheer you up & \\
\hline \multicolumn{2}{|l|}{ Independent Variables } \\
\hline \multicolumn{2}{|l|}{ Sex } \\
\hline What is your sex? A. Female, B. Male & 1 if $\mathrm{A}$; 0 if $\mathrm{B}$ \\
\hline \multicolumn{2}{|l|}{ Disability } \\
\hline $\begin{array}{l}\text { Has a doctor, nurse, or other professional ever told you that you have } \\
\text { one or more of the following: }\end{array}$ & $\begin{array}{l}1 \text { if yes to } C, D \text {, or } F \text {; } \\
0 \text { if yes to } A, B \text { or } E \text {. }\end{array}$ \\
\hline $\begin{array}{l}\text { Are you limited in any activities because of ANY disabilities or long- } \\
\text { term health problems, including physical health, emotional, or } \\
\text { learning problems? A. Yes, B. No, C. Not Sure }\end{array}$ & 1 if $\mathrm{A} ; 0$ if $\mathrm{B}$ or $\mathrm{C}$ \\
\hline \multicolumn{2}{|l|}{ Race/Ethnicity } \\
\hline Are you Hispanic or Latino? A. Yes, B. No & 1 if $A, 0$ if $B$ \\
\hline $\begin{array}{l}\text { What is your race? (Select one or more responses) A. American } \\
\text { Indian or Alaska Native, B. Asian, C. Black or African American, D. } \\
\text { Native Hawaiian or other Pacific Islander, E. White }\end{array}$ & $\begin{array}{l}1 \text { if } \mathrm{A}, \mathrm{B}, \mathrm{C}, \mathrm{D} \text {; } \\
\quad 0 \text { if } \mathrm{E} \text {. }\end{array}$ \\
\hline \multicolumn{2}{|l|}{ Sexual Orientation ${ }^{\mathrm{e}}$} \\
\hline $\begin{array}{l}\text { Which of the following best describes you? A. Heterosexual } \\
\text { (straight), B. Gay or lesbian, C. Bisexual, D. Not sure }\end{array}$ & $\begin{array}{l}1 \text { if } \mathrm{B}, \mathrm{C} \text {, or } \mathrm{D} \text {; } \\
0 \text { if } \mathrm{A}\end{array}$ \\
\hline $\begin{array}{l}\text { During your life, with whom have you had sexual contact? A. I have } \\
\text { never had sexual contact, B. Female, C. Males, D. Females and } \\
\text { Males }\end{array}$ & $\begin{array}{l}1 \text { if same sex } \\
\text { contact; } 0 \text { if not }\end{array}$ \\
\hline
\end{tabular}

Note. Source: 2008 Oregon Healthy Teen survey (Oregon Department of Human Services, 2008).

Response items are italicized.

${ }^{a}$ Preceding explanation for the question about peer victimization included following text:

Harassment can include threatening, bullying, name calling or obscenities, offensive notes or graffiti, unwanted touching, and physical attacks. ' (Select one or more responses.) A. Harassment about your race or ethnic origin, B. Unwanted sexual comments or attention, C. Harassment because someone thought you were gay, lesbian or bisexual, D. Harassment about your weight, clothes, acne, or other physical characteristics, E. Harassment about your group of friends, F. Other reasons, G. I have not been harassed. ${ }^{\mathrm{c}}$ All of the time, B. Most of the time, C. A good bit of the time, D. Some of the time, E. A little of the time, F. None of the time. '(Please mark all that apply) A. I do not have any of these conditions, B. A medical condition lasting more than a year, such as asthma, diabetes, cancer, heart problems or seizures, C. A physical condition, including developmental conditions (spina bifida, cerebral palsy, etc.), long term, injuries (spinal cord injury, etc.), or bone, joint, or muscle problems (arthritis, etc.), D. Blindness or problem seeing (other than needing glasses or contacts) or deafness or problem, Hearing, E. An emotional condition such as depression or anxiety, F. A learning disorder, attention deficit disorder, ADHD, or severe learning disability such as mental, retardation. ${ }^{\mathrm{e}}$ Additional coding was done taking into account the sex of the respondent. 
Disability. Two disability questions were located in the section of the OHT survey under "other health conditions," on page three of the 14-page survey. The first question asked students whether they had ever been told by a "doctor, nurse, or other professional" that they had a medical, physical, sensory, emotional or learning disability. Nearly a third of the students reported that they had been told they had at least one of these conditions (Table 10). Since psychosocial distress is a dependent variable, students who indicated they were told they had an emotional condition (only) were not categorized as disabled. Further, as the study was intended to examine the association of disability status as a marker of exposure to peer victimization status, I did not categorize as disabled those students who reported having been told of (only) having a medical condition, as it is unclear that their disability would be known to others. The same could be said for those with learning disabilities, but since it is plausible that students with learning disabilities would be more likely to require educational accommodations, a decision was made to retain this group of students. The second disability-related question asked students if they were limited in any activities due to a disability or health problem. The percentage of students answering "yes" to this question was about half $(9.6 \%, n=$ 691) of the percentage of students indicating a physical, sensory and/or learning disability $(19.4 \%, n=1343)$ (Table 10). Those answering yes either to the question about having an activity limitation, or to having been told that they had physical, sensory and/or learning disability, were categorized as "disabled" for the purposes of this study. Participants coded as "missing" on the final disability status variable were excluded from all analyses $(n=116)$. 
Sexual orientation. The 2008 OHT survey included two questions relating to sexual identity and sexual contact in the section dealing with "sexual behavior." The first question asked about sexual orientation identity (as heterosexual/straight, gay or lesbian, bisexual, or not sure); $7.6 \%$ of students answering this question indicated being LGB or not sure. The second question asked about sexual contact (behavior). While a small percentage of students $(8.8 \%)$ reported having sexual contact with females and males (Table 10), 37.6\% of students answering this question reported that they had not had sexual contact. I created the sexual orientation variable based upon responses to these two different questions, one involving sexual orientation identity, and the other, sexual behavior. Participants coded as "missing" on the final sexual orientation variable were excluded from intersectional analyses involving sexual orientation as an independent variable. I realize that creating the sexual orientation variable based upon responses to these two questions may seem problematic; however, I chose to do this based upon a recent study conducted by Friedman and colleagues (Friedman et al., 2011). In their study, which also involved the OHT dataset, they found that the dimension used to categorize sexual orientation status (e.g., behavior, identity) did not produce significant differences in capturing the association between sexual orientation and peer victimization. Below I describe my process for coding the final sexual orientation variable.

In regards to sexual orientation identity, research suggests that students who identify as gay, lesbian or bisexual (with or without same sex contact) are at greater risk for exposure to peer victimization compared to students who identify as heterosexual 
(Kosciw et al., 2010). However, not all $11^{\text {th }}$ graders who reported having same sex experience may identify as LGBQ. Indeed, many may report their identity as "questioning." I decided to code "questioning" students as LGBQ based on an assumption that students identifying as "not sure," with or without same sex contact, are likely to be a more visible target, particularly if they are not identifying as heterosexual. In other words, it is plausible that "questioning" students are at greater risk of exposure to peer victimization.

In considering the question about sexual behavior, it is possible that heterosexually-identified students with same sex experience are not at greater risk of exposure to peer victimization, as the meaning associated with identity (as heterosexual) "may be more important than the acts themselves" and confer "upon heterosexual individuals all sorts of citizenship rights" (Pascoe, 2011, p. 10). Therefore I excluded heterosexually identified students who reported having had same sex contact from analyses $(n=360,5.7 \%)$, in an effort to keep the final sexual orientation variable as "clean" as possible.

Race/ethnicity. Race and ethnicity demographics were collected separately with two questions, making it possible to distinguish, for example, non-Latino white students from Latino white students. The first question asked students "Are you Hispanic or Latino?" Of those who answered this question, $84.2 \%$ reported that they were not Hispanic or Latino. The second question gave students a choice of racial categories, of which they could choose more than one (American Indian or Alaska Native, Asian, Black or African American, Native Hawaiian or Other Pacific Islander, and White). Nearly 20\% 
of the students answering this question self-identified as students of color. Only students who did not answer both questions, or answered "White" but not the question about being Hispanic or Latino, were excluded from a single composite race/ethnicity variable I created $(n=123,1.84 \%)$. This coding classification allowed me to retain a greater number of Latino students who had not answered the second question about race.

While it is preferable to have greater specificity in the use of racial/ethnic categories, given the relatively large rate of missing responses to the second question about race $(n=675,9.6 \%$, Table 10$)$, and the relatively small numbers of students of color with disabilities $(n=373,5.8 \%$, Table 11$)$, I collapsed all the racial/ethnic categories into two categories with students of color and/or Latino in one category, and white, non-Hispanic students in another category. Therefore, for the purposes of this study, "students of color" includes Hispanic/Latino youth of any race. White students who did not answer the question about ethnicity, and non-Hispanic students who did not answer the question about race were coded as "missing" on the final race/ethnicity variable.

Sex. Respondents were asked "What sex are you?" with a choice of male or female. Sex (male/female) was treated as a control variable or as an independent variable in the intersectional analyses, and as a moderator in the moderated mediation analyses. 
Table 10

Counts and Weighted Percentages by Selected Key Study Variables

\begin{tabular}{|c|c|c|c|}
\hline & $N$ & $M / \%$ & $S E$ \\
\hline \multicolumn{4}{|l|}{ Dependent and Mediator Variables } \\
\hline MHI5 Score (scaled 0 to 100 ) & 6822 & 71.5 & 0.2 \\
\hline Peer victimization $(\mathrm{PV})(n=6718)$ & 2048 & 30.58 & 0.8 \\
\hline \multicolumn{4}{|l|}{ Independent Variables } \\
\hline $\operatorname{Sex}(N=7091)$ & 3662 & 51.5 & 0.01 \\
\hline \multicolumn{4}{|l|}{ Disability status (was told by health professional that I have....) $(n=6875)$} \\
\hline Not been told have any of these conditions & 4336 & 62.9 & 0.69 \\
\hline Medical condition & 1008 & 14.7 & 0.57 \\
\hline Emotional condition & 915 & 13.3 & 0.39 \\
\hline Physical condition & 650 & 9.4 & 0.31 \\
\hline Severe hearing or vision condition & 274 & 4.1 & 0.26 \\
\hline Learning disability (LD) and/or ADHD ${ }^{\text {a }}$ & 597 & 8.6 & 0.44 \\
\hline Total: Physical, sensory and/or $L D / A D H D$ & 1343 & 19.4 & 0.64 \\
\hline \multicolumn{4}{|l|}{ Have an activity limitation due to disability/long term problem? $(n=6930)$} \\
\hline Yes & 691 & 9.6 & 0.35 \\
\hline No & 5787 & 83.7 & 0.45 \\
\hline Not sure & 452 & 6.8 & 0.38 \\
\hline \multicolumn{4}{|l|}{$\begin{array}{l}\text { Final disability variable (activity limitation and/or physical, } \\
\text { sensorv or LD/ADHD condition }(n=6975)^{a b}-\text { Disabled students }\end{array}$} \\
\hline \multicolumn{4}{|l|}{ Race and Ethnicity (combination of two questions) $(n=6968)$} \\
\hline Non-Hispanic, White & 5137 & 71.8 & 0.02 \\
\hline Latino or student of color ${ }^{b}$ & 1016 & 15.7 & 0.02 \\
\hline Latino AND student of color & 198 & 3.7 & 0.01 \\
\hline Student of color - unknown ethnicity & 28 & 0.5 & 0.00 \\
\hline Latino-unknown race & 589 & 8.4 & 0.01 \\
\hline Final race/ethnicity variable $(n=6968)^{b}$ & 1831 & 28.2 & 0.02 \\
\hline \multicolumn{4}{|l|}{ Sexual identity $(n=6674)$} \\
\hline Heterosexual (straight) & 6207 & 92.4 & 0.59 \\
\hline Gay or lesbian & 75 & 1.3 & 0.15 \\
\hline Bisexual & 244 & 3.7 & 0.29 \\
\hline Not sure & 148 & 2.5 & 0.25 \\
\hline \multicolumn{4}{|l|}{ Sexual contact (with whom?) $(n=6632)$} \\
\hline Never had sex & 2429 & 37.6 & 0.79 \\
\hline Females & 1959 & 28.9 & 0.61 \\
\hline Males & 1939 & 28.6 & 0.54 \\
\hline Females and Males & 305 & 4.9 & 0.33 \\
\hline Yes-same sex contact (based upon responses and sex of student) & 554 & 8.8 & 0.52 \\
\hline Final sexual orientation variable $(n=6227)^{\text {bd }}$ & 450 & 7.85 & 0.01 \\
\hline
\end{tabular}

${ }^{\mathrm{a}} \mathrm{ADHD}=$ Attention deficit hyperactivity disorder; $\mathrm{LD}=$ learning disability. ${ }^{\mathrm{b}}$ Dichotomous variable.

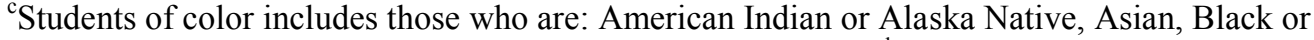
African American, Native Hawaiian or Other Pacific Islander. ${ }^{\mathrm{d}}$ Heterosexual students with same sex experience were excluded from the final variable. ${ }^{e} \mathrm{LGBQ}=$ Lesbian, gay, bisexual or questioning. 


\section{Intersectional Independent Variables}

As analyses for research questions 2.1 to 2.3 were based upon an inter-categorical intersectional approach, I intentionally avoided the use of interaction terms. The danger of interaction terms is summarized by Hancock (2007), who explains that linear regressions attempting to "capture the simultaneous impact of race and gender (are) multiplicative in that (they) test whether race, gender, or race and gender provide the greatest explanatory power" (2007, p. 70). Thus, instead of creating interaction terms, I created new intersectional variables based upon the combination of two or more social statuses. By doing so, I adhered to an inter-categorical approach as described by (McCall, 2005) and the methodological suggestion of Sen, Iyer and Mukherjee (2009). This approach allowed an examination of the relative impact of disability status, in combination with other marginalized statuses, on exposure to peer victimization among $11^{\text {th }}$ graders in Oregon. I provide a description of the intersectional variables created next. These intersectional variables were constructed as categorical variables; I was able to utilize a feature in Stata that converted these categorical variables to dummy variables when running logistic tests. Table 11 provides the count and percentage of these intersectional variables.

Disability and sex. A categorical variable was created representing the combined intersectional variable of disability and sex, with non-disabled males coded as 0 (referent group), followed by non-disabled females (coded as 1), disabled males (coded as 2), and disabled females (coded as 3). 
Disability and race/ethnicity. A categorical variable was created representing the combined intersectional variable of disability and race/ethnicity, with non-disabled white, non-Hispanic youth coded as 0 (referent group), followed by disabled white, nonHispanic youth (coded as 1), non-disabled students of color (coded as 2), and disabled students of color (coded as 3).

Disability and sexual orientation. A categorical variable was created representing the combined intersectional variable of disability and sexual orientation, with non-disabled heterosexuals (without same-sex contact) coded as 0 (referent group), followed by disabled heterosexuals (coded as 1), non-disabled LGBQ students (coded as 2), and disabled LGBQ students (coded as 3).

Disability, sex, and race/ethnicity. A categorical variable was created representing the combined intersectional variable of disability and race/ethnicity, with non-disabled white, non-Hispanic males coded as 0 (referent group), followed by nondisabled white, non-Hispanic females (coded as 1), non-disabled male students of color (coded as 2), non-disabled female students of color (coded as 3), disabled white, nonHispanic males (coded as 4), disabled white, non-Hispanic females (coded as 5), disabled male students of color (coded as 6), and disabled female students of color (coded as 7).

Disability, sex, and sexual orientation. A categorical variable was created representing the combined intersectional variable of disability, sex and sexual orientation, with non-disabled heterosexuals males (without same-sex contact) coded as 0 (referent group), followed by disabled heterosexuals males (coded as 1), non-disabled 
Table 11

Counts and Weighted Percentages of Intersectional Variables

\begin{tabular}{lccc}
\hline & $N$ & $\%$ & $S E$ \\
\hline Disability and sex subgroups & 6975 & & \\
Non-disabled male & 2600 & 37.8 & 0.52 \\
Non-disabled female & 2683 & 38.6 & 0.68 \\
Disabled male & 754 & 10.5 & 0.39 \\
Disabled female & 938 & 13.2 & 0.40 \\
Disability and race/ethnicity & 6858 & & \\
Non-disabled, white and non-Latino & 3791 & 54.3 & 1.73 \\
Non-disabled, person of color and/or Latino & 1405 & 22.1 & 2.09 \\
Disabled, white and non-Latino & 1289 & 17.9 & 0.84 \\
Disabled, person of color and/or Latino & 373 & 5.8 & 0.33 \\
Disability and sexual orientation & 6209 & & \\
Non-disabled heterosexual & 4461 & 71.7 & 0.59 \\
Disabled heterosexual & 1301 & 20.5 & 0.83 \\
Non-disabled LGBQ & 278 & 5.2 & 0.50 \\
Disabled LGBQ & 169 & 2.7 & 0.20 \\
Disability, race and sex & 6858 & & \\
Non-disabled, white (non-Latino), male & 1835 & 26.4 & 0.88 \\
Non-disabled, white (non-Latino), female & 1956 & 27.9 & 1.01 \\
Non-disabled, person of color and/or Latino, male & 711 & 11.2 & 0.92 \\
Non-disabled, person of color and/or Latino, female & 694 & 10.9 & 1.24 \\
Disabled, white, male & 567 & 7.9 & 0.42 \\
Disabled, white, female & 722 & 10.0 & 0.48 \\
Disabled, person of color and/or Latino, male & 170 & 2.6 & 0.16 \\
Disabled, person of color and/or Latino, female & 203 & 3.2 & 0.23 \\
Disability, sex and sexual orientation & 6209 & & \\
Non-disabled, heterosexual, male & 2250 & 36.4 & 0.62 \\
Disabled, heterosexual, male & 587 & 9.2 & 0.50 \\
Non-disabled, heterosexual, female & 2211 & 35.3 & 0.62 \\
Disabled, heterosexual, female & 714 & 11.3 & 0.45 \\
Non-disabled LBQ, female & 176 & 3.3 & 0.39 \\
Disabled LBQ, female & 111 & 1.8 & 0.18 \\
Non-disabled GBQ, male & 102 & 1.9 & 0.24 \\
Disabled GBQ, male & 58 & 0.8 & 0.09 \\
\hline
\end{tabular}

Note. $N=7091$. $\mathrm{L}=$ lesbian, $\mathrm{G}=$ gay, $\mathrm{B}=$ bisexual, $\mathrm{Q}=$ questioning or not sure. 
heterosexuals females (coded as 2), disabled heterosexuals females (coded as 3), nondisabled LGBQ males (coded as 4), disabled LGBQ females (coded as 5), non-disabled LGBQ males (coded as 6), and disabled LGBQ males (coded as 7).

\section{Analyses}

I conducted preliminary analyses of all relevant variables in the data set, including the weighted variable created by the OHA staff, as recommended by Heeringa, West and Berglund (2010a). As this dataset involves complex survey data, standard errors could be biased, unless the correct estimate of the variance is utilized. One of three estimate options in Stata is the Taylor series linearization (TSL) method, which is appropriate for analyses with the type of complex data present in this study (Heeringa et al., 2010a). This option takes into account the complex design factors, such as stratification and weighting (Heeringa et al., 2010a). Statistical testing in this study, using syntax supported by Stata survey commands, utilizes methodology to account for the sampling design with output that is similar to that of an $F$ test, $t$-test, and other statistical tests; this is often referred to a design-based (adjusted) statistic. Excluding frequencies, all statistics reported related to this research based upon the OHT dataset are weighted estimates taking into account the complex survey design. As recommended, instead of the Pearson $X^{2}$, the Rao-Scott correction $F$-statistic was used, and the Wald method was used to produce an adjusted design-based $F$ test in regression analyses as well as tests of differences between subgroups in the logistic model (StataCorp, 2011). The design-based goodness-of-fit was estimated using a $F$-adjusted mean residual test (Archer, Lemeshow, \& Hosmer, 2007). As the reader may notice later, the denominator for the degrees of freedom associated 
with the $F$ statistic is small given the size of the study sample. Every design-based test will have a different methodology for calculating the degrees of freedom. In the case of the adjusted design-based $F$ test in regression analyses utilizing the Wald method, the numerator consists of the number of predictors (without the intercept), and the denominator degrees of freedom equals: \# of PSUs - \# of stata - \# of predictors +1 (StataCorp, 2011). In the case of this study, the overall design degree of freedom (denominator) was 68 (76 school districts -8 regions).

Below I describe the steps taken to assess the adequacy of the data set overall, starting with missing data checks and missing patterns, followed by the steps taken to examine the assumptions, and analyses conducted.

\section{Missing Data}

Because thorough data quality checks for case-level missingness as well as checks for suspect responses were conducted by OHA, I focused upon variable-level missingness to determine which variable responses had more than a $2 \%$ rate of missing values. The rate of missing responses was particularly high for sensitive items, particularly in regards to sexual orientation. The sexual contact question had a higher rate of missingness $(6.4 \%$, $n=459)$, compared to the question in regards to sexual identity $(5.6 \%, n=417)$, resulting in a missing rate of $7.1 \%$ for the final variable. This may be due to the nature of sexual identity and behavior being very personal, private, and potentially stigmatizing. Social desirability bias may have accounted for a nearly $5 \%$ non-response rate to the question about harassment (Table 12). 
My examination of the missingness pattern, using Stata's misspattern command, did not reveal a monotonic pattern of missingness; rather it was arbitrary in nature, ruling out MNAR (missing not at random). Examination of missing patterns with the key independent variables (disability, race/ethnicity, sexual orientation) and dependent variable (peer victimization) together revealed that of the 504 missing responses on sexual orientation, more than half the same respondents also were missing on peer victimization ( $n=299,3.76 \%$ ). A deeper examination revealed significantly higher rates of missing responses on sexual identity by disability status $[F(1,68)=7.816, \mathrm{p}=.007]$, and by race/ethnicity $[F(1,68)=10.743, \mathrm{p}=.002]$. Over six percent of disabled youth $(6.2 \%)$ did not answer the question on sexual identity, compared to $4.2 \%$ of non-disabled youth. The weighted percentage of youth of color not answering the sexual identity question was almost twice that of white students not answering the same question $(8.7 \%$ and $4.4 \%$ respectively).

Significant differences in non-responses to the sexual contact questions were also found by disability status, race/ethnicity and exposure to peer victimization. Compared to non-disabled youth $(5 \%)$, disabled youth $(7.1 \%)$ were significantly more likely not to answer the question about sexual contact $[F(1,68)=8.49, \mathrm{p}=.005]$. A significantly higher percentage of students of color did not answer the sexual contact question $(9.3 \%)$, compared to white, non-Hispanic students $[5.2 \%, F(1,68)=10.564, \mathrm{p}=.002]$. The pattern of higher rates of missing responses by students of color is consistent with a recent article based upon the 2006-2008 National Survey of Family Growth of males and females age 15 to 44 (Chandra, Mosher, Copen, \& Sionean, 2011). 


\section{LOST IN THE MARGINS}

Table 12

Weighted Percentages and Counts of Missingness among Key Study Variables

\begin{tabular}{lcc} 
Variable & \multicolumn{2}{c}{ Missing } \\
\hline Sexual orientation $^{\mathrm{a}}$ & $\%$ & $n$ \\
Peer victimization & 7.1 & 504 \\
Psychosocial distress (MHI-5 score) $^{\text {Race/ethnicity }}$ & 4.8 & 373 \\
Disability & 3.5 & 269 \\
${ }^{\mathrm{a}}$ Did & 1.8 & 123 \\
\end{tabular}

${ }^{\mathrm{a}}$ Did not include heterosexually-identified students reporting same sex contact. 
Significant differences in non-responses to the sexual contact questions and peer victimization were found. Youth reporting peer victimization (4\%), compared to youth not reporting peer victimization $(2.5 \%)$ were significantly less likely to answer the sexual contact question $[F(1,68)=12.406, \mathrm{p}=0.001]$. To examine the possibility that those not answering the sexual contact question might in fact be more likely to report victimization, I conducted logistic regression with peer victimization as the dependent variable, using a categorical variable with 9 subgroups (eight of the subgroups were derived from different combinations of sexual contact and sexual behavior, such as "questioning, no same sex"). Compared to non-responders (reference group), heterosexuals not reporting same sex experience were less likely $(O R=0.6[4.8,7.4])$, while lesbian or gay $(O R=10.1[4.56$, $22.5])$, and bisexual students $(O R=2.4[1.6,3.4])$, reporting same sex experience were more likely to report exposure to peer victimization. However, significant differences in exposure to peer victimization between non-responders and questioning students (with or without same sex experience), and bisexual or gay students not reporting same sex experience were not detected.

The differential patterns in the odds of exposure to peer victimization, with nonresponders either being less likely to report peer victimization compared to some of the subgroups, and more likely to report peer victimization compared to other subgroups, suggest that while there is a non-response bias, it is likely not representative of bias due to a particular sexual identity or behavior not reported. Nonetheless, the non-response bias created a limitation in generalizing the results of this study, as the non-response in regards to sexual orientation status on peer victimization is not negligible. I was not able 
to assume MAR (missing at random), nor was I able to control the non-response bias by controlling other variables (Groves, 2006).

\section{Part 1: Analyses (Research Questions 1.1 to 2.3)}

This section provides additional detail on the analyses conducted to answer the following research questions related to the intersectional analyses.

Assumptions. The multivariate analyses involved logistical regression, which is suited to testing hypotheses with the categorical independent variables (intersectional social statuses) and a dichotomous dependent variable(peer victimization) (Peng \& So, 2002). As this is a randomized, representative sample, and all students were represented in the dataset only once, the assumption of a binomial distribution can be assured (Peng \& So, 2002). A nonlinear relationship is assumed in logistic regression; in this study as all study variables were categorical, this assumption was met. As the intersections of identities were handled via a coding scheme to ensure that each observation shows up in only one predictor category at a time, I was able to avoid perfect multicollinearity among predictors, which is a required assumption for logistic regression (Menard, 2010). While there are no theoretical or empirical grounds to expect high collinearity among the group status predictors, I checked multicollinearity among sex, disability, race/ethnicity and sexual orientation status. The results suggest no problems with multicollinearity as the tolerance for each variable was above 0.98 . Another required assumption for logistic modeling is that the model included all relevant predictors, and excluded extraneous variables (Menard, 2010). However, as noted by Wright (1994), "in practice...the specificity assumption is rarely met" (p.220). In this research study, not all possible 
relevant predictors of peer victimization were included in the survey design. For example, predictors measuring the degree of non-conformity among all social groups would strengthen the model. A link test was done following logistical testing, with peer victimization as the dependent variable for each model to rule out other significant predictors of peer victimization not in the model.

Univariate and bivariate statistics. Univariate statistics were conducted with attention to rate of missing values (as described above). Bivariate analyses were conducted to examine the association between selected characteristics such as sex, disability, race, sexual orientation, and reporting peer victimization. As explained in the section on measures, the measures used in these analyses were based upon an intercategorical intersectional approach, with a series of categorical variables constructed from the combination of two or more social statuses. Testing was done to determine if there are significant differences between groups reporting peer victimization.

Multivariate statistics. Six binominal logistic regressions were conducted, with peer victimization as the dependent variable (Models 1 through 6). As all independent variables indicate statuses, and utilizing a feature in Stata that converted the categorical variables to dummy variables when running logistic tests, I reported the odds ratio which lends itself to an intuitive, accessible interpretation (Menard, 2010). The first regression (Model 1) conducted tested for the independent effects of sex, disability, sexual orientation and race/ethnicity on exposure to peer victimization (dependent variable), thereby answering research questions 1.1 (Is disability status associated with peer victimization, and if so, to what extent?). 
Models 2 through 4 addressed research question 2.1. (Taking an intersectional approach, what is the relationship between (a) disability status with each of the three demographic variables - sex, sexual orientation, and racelethnicity, and (b) peer victimization, controlling for other demographic variables?). Specifically, the second model tested the likelihood of exposure to peer victimization by the intersections of disability and sex combined. The third model tested the likelihood of exposure to peer victimization by the intersections of disability, and race/ethnicity combined. The fourth model tested the likelihood of exposure to peer victimization by the intersections of disability and sexual orientation combined.

The fifth model, addressing research question 2.2 (Taking an intersectional approach, what is the relationship between (a) disability status with sex, and race/ethnicity, and (b) peer victimization, controlling for other demographic variables?), tested the likelihood of exposure to peer victimization by the combined intersections of disability, sex, and race/ethnicity. The sixth model, addressing research question 2.3 (Taking an intersectional approach, what is the relationship between (a) disability status with sex, and sexual orientation, and (b) peer victimization, controlling for other demographic variables?), tested the likelihood of exposure to peer victimization by the combined intersections of disability, sex, and sexual orientation.

Care was taken to ensure that the each individual was present in all models only once. Since significant differences in exposure to peer victimization were found by region, all models controlled for region. In addition to regions, sex, disability, race/ethnicity and sexual orientation variables were also controlled for, resulting in 
adjusted odd ratios. The overall goodness of fit for all models was assessed with the Hosmer and Lemeshow's goodness of fit test using the "svylogitgof" command in Stata which is recommended to be used with complex survey data (Archer et al., 2007; Heeringa et al., 2010b). All goodness-of-fit tests indicated satisfactory fit with the inclusion of regions in the models, with the exception of the fifth model. A logistic regression done without region resulted in a satisfactory goodness-of-fitness result. Follow-up tests (adjusted Wald) tested for significant differences between and within subgroups represented in models five and six. Results of the link test were non-significant for each of the six logistic regressions, indicating that other relevant variables in the dataset predicting peer victimization were not omitted (UCLA: Statistical Consulting Group, 2012).

Statistical power. To calculate the detectable odds ratio for the intersections of disability, sex, and race/ethnicity, I relied upon a calculator developed specifically for logistic regression (Demidenko, 2007a, 2007b). Based upon the sample demographics (Table 11), and estimates of peer victimization based upon the literature review (Table 13), I calculated the detectable odds ratio for the two smallest cells - both having to do with disabled youth of color (males and females). When these low cell counts were taken into consideration as the maximum threshold for power calculations, the proportion of cases for disabled males and females of color were calculated to be .027 , and .033 respectively (Table 14). Based upon the premise that being disabled and also a person of color is not protective, I set the prevalence rate of peer victimization to $38 \%$ based upon YRBS results reported in Montana (Montana Office of Public Instruction, 2011). With 
power set to 0.8 , the lowest detectable odds ratio was 1.45 (Table 14). This means the odds ratio needs to exceed 1.45 before being detected as significant.

To calculate the detectable odds ratio for the intersections of disability, sex, and sexual orientation, I repeated these steps, except in this case I included the three smallest cells (Table 15). The proportion of cases for disabled GBQ males, non-disabled GBQ males, and disabled LBQ females were $0.009,0.016$, and 0.018 respectively. Based upon the premise that disability or sexual orientation is not protective when combined, I set the prevalence rate of peer victimization to $55 \%$ based upon OHT results reported by Hatzenbuehler (2011) for bisexual youth (choosing a more conservative percentage). With power set to 0.8 , the lowest detectable odds ratio calculated was 1.63 (Table 14). While an odds ratio of 1.6 is not considered to represent a strong relationship between variables, there is sufficient power to at least determine if there is a significant relationship between variables (Haddock, Rindskopf, \& Shadish, 1998). 
Table 13

Estimates Used for Detectable Effect Size Calculations

\begin{tabular}{|c|c|c|c|c|}
\hline & \multicolumn{2}{|c|}{$\begin{array}{l}\text { Percent reporting } \\
\text { peer victimization }\end{array}$} & \multicolumn{2}{|c|}{ Reported odd ratios } \\
\hline & Male & Females & Male & Females \\
\hline \multicolumn{5}{|l|}{ Disability } \\
\hline YRBS (Montana, 2009) & \multicolumn{2}{|c|}{38} & \multicolumn{2}{|c|}{-} \\
\hline Sentenac (2010) & \multicolumn{2}{|c|}{41} & \multicolumn{2}{|c|}{1.3 to 1.8} \\
\hline \multicolumn{5}{|l|}{ Race/Ethnicity } \\
\hline \multicolumn{5}{|l|}{ Eaton et al. (2010) } \\
\hline Hispanic & 18 & 18.9 & \multicolumn{2}{|c|}{-} \\
\hline Black & 11.9 & 15.5 & & \\
\hline \multicolumn{5}{|l|}{ Sawyer et al. (2008) } \\
\hline Hispanic & 34.5 & 23.2 & 1.67 & ns \\
\hline Black & 20.3 & 12.3 & 0.6 & ns \\
\hline Other & 30.6 & 27.9 & 1.66 & 1.64 \\
\hline \multicolumn{5}{|l|}{ Sexual minority } \\
\hline \multicolumn{5}{|l|}{ Hatzenbuehler (2011) } \\
\hline Lesbian / Gay & \multicolumn{2}{|c|}{58.8} & \multicolumn{2}{|c|}{-} \\
\hline Bisexual & \multicolumn{2}{|c|}{55.8} & \multicolumn{2}{|c|}{-} \\
\hline \multicolumn{5}{|l|}{ Berlan et al. (2010) } \\
\hline Gay / Lesbian & 43.6 & 40 & 1.98 & 3.36 \\
\hline Bisexual & 35.7 & 25.6 & 1.46 & 1.63 \\
\hline
\end{tabular}

Note. Dash $(-)=$ not reported, $\mathrm{ns}=$ not significant. All figures come from the empirical literature review reported in Tables 1,2 and 3. 
Table 14

Detectable Effect Size Calculations for Logistical Regressions

\begin{tabular}{|c|c|c|c|c|}
\hline & $\mathrm{N}$ & $\operatorname{Prx}$ & Pry & $\begin{array}{l}\text { Detectable } \\
\text { odds ratio }\end{array}$ \\
\hline \multicolumn{5}{|c|}{ Intersections of Disability, Sex, and Race/ethnicity } \\
\hline Disabled males color and/or Latino & 170 & $0.38^{\mathrm{a}}$ & 0.027 & 1.529 \\
\hline Disabled females of color and/or Latino & 203 & $0.38^{\mathrm{a}}$ & 0.033 & 1.447 \\
\hline Total sample size & 6209 & & & \\
\hline \multicolumn{5}{|c|}{ Intersections of Disability, Sex, and Sexual Orientation } \\
\hline Disabled GBQ males & 58 & $0.55^{\mathrm{b}}$ & 0.009 & 1.95 \\
\hline Non-disabled GBQ males & 102 & $0.55^{\mathrm{b}}$ & 0.016 & 1.68 \\
\hline Disabled LBQ females & 111 & $0.55^{\mathrm{b}}$ & 0.018 & 1.63 \\
\hline Total sample size & 6209 & & & \\
\hline
\end{tabular}

Note. $\mathrm{G}=$ gay, $\mathrm{B}=$ bisexual, $\mathrm{q}=$ questioning or "not sure"; $\mathrm{L}=$ lesbian. Estimates derived from Demidenko (2007) power calculator for logistic regression with binary covariates with alpha $=.05$, power $=0.80$. Prx $=$ estimated proportion of cases reporting peer victimization. Pry $=$ proportion of cases (group of interest) in the total sample.

${ }^{a}$ Estimates based upon Montana YRBS results (Montana, 2011). ${ }^{b}$ Estimates based upon study results by Hatzenbuehler (2011). 


\section{Part 2: Analyses (Research Question 3.1 to 3.3)}

This section provides additional detail on the bivariate and multivariate analyses conducted to answer the related the mediation analyses.

Assumptions. As pointed out by Judd and Kenny (2010), establishing the causal assumptions underlying a mediation model are extremely difficult, and "ultimately, theoretical and empirical arguments need to be made for the plausibility of a mediation process" (p.119). I briefly discuss the plausibility of the mediation model before describing in greater detail the methodology used for this study. First, it is highly plausible that disability status occurred or existed previous to the onset of exposure to peer victimization, thus meeting a required assumption of mediation. Based upon the empirical literature review in Chapter Two, it is likely that disability status predicts both exposure to peer victimization and increased psychosocial distress. Empirical and theoretical literature reviewed above also suggests it is plausible that peer victimization mediates the relationship between disability status and psychosocial distress. In terms of moderation, based upon the literature review, it is possible that sex moderates the mediating effects of peer victimization on psychosocial distress. Thus, analyses involved both testing mediation, as well as moderated mediation (see Figure 2). Admittedly, it is possible that there is an element of non-recursiveness in the model with psychosocial distress creating greater vulnerability to peer victimization. Nonetheless, based on both empirical evidence and theory, the moderated mediated model is plausible. The validity of the statistical models utilized is described below in Chapter Five of this dissertation. 
Univariate and bivariate statistics. Preliminary analyses were conducted to examine sample characteristics. In preparation for regression, descriptive statistics were calculated for the subpopulation of disabled students who answered both the peer victimization question, and at least four of the five MHI questions. Tests were done to determine if there were significant differences by disability status and peer victimization, and significant differences in mean MHI scores by disability status.

Multivariate analyses. Analyses began with testing for mediation as a starting step (Judd \& Kenny, 2010), involving four steps (Baron \& Kenny, 1986; Kenny, 2011). A series of regressions were conducted separately for males and females which made it possible to determine if sex moderated the mediated effect of peer victimization on psychosocial distress (MacKinnon, Fairchild, \& Fritz, 2007).

Research question 3.1. The first step of the Baron and Kenny (1986) approach to testing mediation addressed research question 3.1., which was to determine whether and the extent to which disability status was associated with psychosocial distress. Linear regressions, to determine the total effect (path c) of disability status on psychosocial distress (MHI-5 total score), were conducted for male and females separately (Figure 2a).

Research question 3.2. The second step in the Baron and Kenny approach (Baron \& Kenny, 1986; Kenny, 2011) involved testing to determine the extent to which disability status is associated with peer victimization (path a) (Figure 2b). Peer victimization was regressed on disability status using logistic regression for males and females separately, as the peer victimization measure was dichotomous. The odds ratios 


\begin{tabular}{|c|c|c|}
\hline $\begin{array}{c}\text { Disability status } \\
\text { (Independent Variable - X) }\end{array}$ & c & $\begin{array}{c}\text { Psychosocial distress } \\
\text { (Dependent Variable - Y) }\end{array}$ \\
\cline { 2 - 3 }
\end{tabular}

a). Direct Pathway

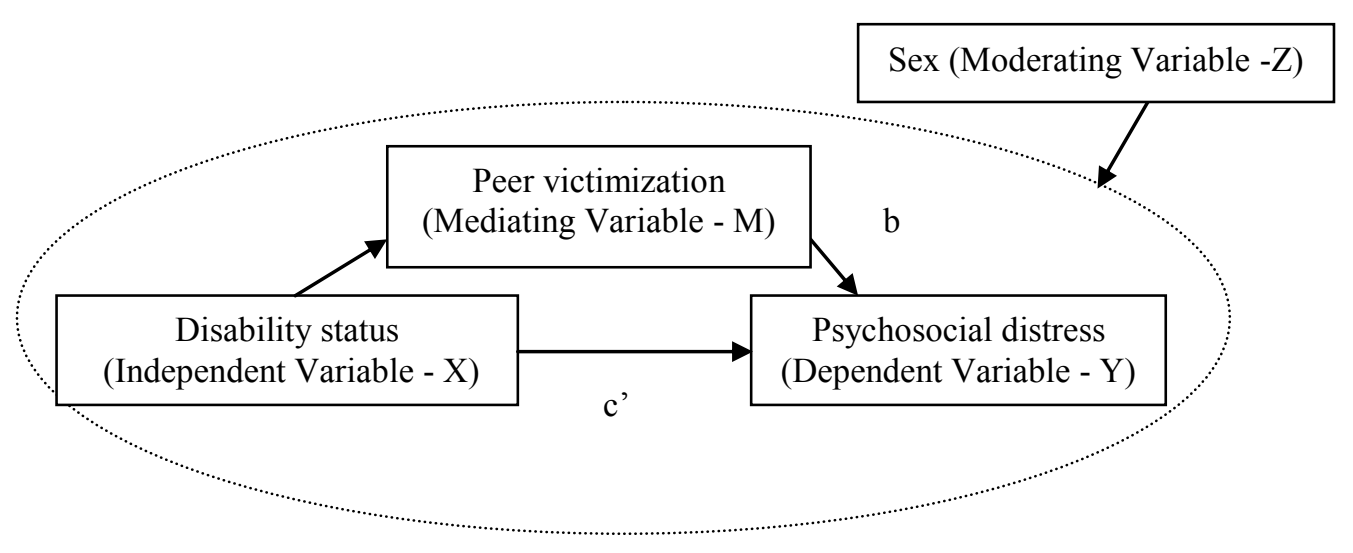

b). Moderated Mediated Pathway

Figure 2.

Moderated mediation model (with subpopulations of males and females regressed separately). 
from this regression provided a measure of the effect of disability status on exposure to peer victimization for both males and females.

The third and final step involved testing the effect of the mediator (peer victimization) on psychosocial distress (path b), controlling for disability status, as well as the effect of disability status on psychosocial distress (path c'), controlling for exposure to peer victimization using linear regression (Figure 2b). Assumptions of normality and linearity were then examined in order to determine the appropriateness of the model.

In order to statistically establish mediation the direct relationship between the predictor (disability status) and the dependent variable (psychosocial distress) must change after controlling for the mediator (peer victimization), or in other words, $\mathrm{c}-\mathrm{c}$ ' does not equal zero (Judd \& Kenny, 2010). Full mediation is indicated when the regression coefficient for path c' is non-significant, or is zero (Judd \& Kenny, 2010). Partial mediation is indicated by a significantly reduced path c' compared to path c (i.e., path c > path c').

The degree to which peer victimization significantly mediates the relationship between disability status and psychosocial distress is indicated by the indirect effect, which is not directly provided by the three steps above. Typically this is done by either utilizing the "product of coefficients" method (path a • path b, or ab), or the "difference of coefficients" method (c - c'). Typically both methods (ab, c - c') produce the same results, however, this is not the case when utilizing different samples, or when the coefficients are scaled differently (MacKinnon, 2008). However, if the coefficient 
resulting from the logistic regression is standardized, both methods should be similar (Herr, n.d.; Kenny, 2008, 2011; MacKinnon, 2008)

Following the steps recommended by Herr (n.d.) and Kenny (2008, 2011), I

computed the mediated effect using the product method $(\mathrm{ab})$ via a three-step process. The first step required determining variance of the mediator (M'), which was done by utilizing the following equation (Herr, n.d.; Kenny, 2008, 2011,) in which "a" represents the unstandardized coefficient estimate for path a and $\mathrm{X}$ is disability status:

$$
\operatorname{Var}\left(M^{\prime}\right)=a^{2} \cdot \operatorname{Var}(X)+\left(\pi^{2}\right) / 3
$$

I then computed the standardized path coefficient which was done by multiplying the unstandardized coefficient of path a by the standard deviation of the predictor variable (X, disability status) divided by the standard deviation (SD) of the mediator variable (M'):

\section{Standardized path a coefficient $=$ path a coefficient $\bullet\left[S D(X) / S D\left(M^{\prime}\right)\right]$}

Once I had the standardized path a coefficient, I was then able to calculate the mediated effect, utilizing the product method (standardized path a coefficient $\bullet$ unstandardized path $b$ coefficient).

As normality cannot be assumed with a dichotomous mediator, the SobalGoodman mediation test is not recommended (Herr, n.d.). Typically bootstrapping is recommended (Preacher, Rucker, \& Hayes, 2007) to estimate the variance of the indirect effect; however, due to the challenges of testing mediation with a dichotomous mediator, with complex survey data in Stata, I opted instead to compute the confidence interval of 
the mediated effect $(\mathrm{ab})$. The equation suggested by MacKinnon to compute the upper and lower limits of the confidence interval is:

Upper/lower confidence interval $=$ mediated effect $\pm 1.96($ SE of ab estimate $)$

Research question 3.3. To determine if sex moderated the mediation effects of peer victimization on psychosocial distress, the direct and indirect effects, resulting from the two mediation analyses done separately for the males and females, were compared, representing a fairly straightforward test of moderated mediation (MacKinnon et al., 2007).

Statistical power. Prior to conducting the planned analyses in this section, I estimated detectable effect size needed. Since the research involving moderated mediation requires comparing the results of the path's strengths between peer victimization and psychosocial distress separately for males and females, it is important to ensure adequate sample size for both groups. Based upon power tables supplied by Fritz and MacKinnon (Fritz \& MacKinnon, 2007), with a power of .80 and alpha of .05, the minimum sample size required to test mediation (utilizing the bootstrap procedure), assuming conservatively a small effect size and small direct effect, was 558, which is well within the sample size of 754 males, and 938 females with disabilities. 


\section{CHAPTER V: RESULTS}

The scaling and general distribution of the weighted variables met assumptions of normality without extreme weight values assigned by the OHA staff to a case. Slightly more than half of the $709111^{\text {th }}$ graders responding to the survey were female $(51.5 \%, n=$ 3662 ), and the mean age of $11^{\text {th }}$ graders in the randomized sample was 16.7 . Nearly a quarter of the students were categorized as disabled using the single disability composite variable $(n=1692,23.7 \%)$ (Table 10$)$. Overall, nearly a third of the students indicated being Hispanic/ Latino and/or a person of color $(n=1831)$ (Table 10$)$. Over $90 \%$ of the students identified as heterosexual. See Table 11 for the counts and weighted percentages by the intersectional variables. The values of differences between subgroups became markedly different as the number of subgroups were increased from four (e.g., disability and sex) to eight (e.g., disability, sex, and race) subgroups (Table 11). The smallest subgroup consisted of students identifying as disabled, male, and gay, bisexual or questioning $(n=58,0.81 \%)$ (Table 11$)$.

Nearly a third of the students answering the question about peer victimization reported being harassed at school, on the way to/from school, in the past 30 days (Table 15). With the single addition of sex, race/ethnicity, and sexual orientation to disability status, the percentage of students reporting peer victimization increased greatly, particularly for disabled females (42.5\%), disabled students of color (49\%), non-disabled LGBQ students (44.8\%), and disabled LGBQ students (61.4\%) (Table 15). The lowest rate of exposure to peer victimization by two variables combined was found for nondisabled males (25.3\%), followed closely by white (non-Hispanic) males $(25.6 \%)$. 
The combined social variable of disability, sex, and race/ethnicity, revealed varying percentages of students reporting peer victimization, ranging from $22.3 \%$ for non-disabled, white (non-Hispanic) males, to $49 \%$ among disabled males of color. Cell sizes ranged from 1366 (non-disabled white males not reporting peer victimization) to 73 (disabled males of color reporting peer victimization) (Table 15). The combined social variable of disability, sex and sexual orientation also revealed varying percentages of students reporting peer victimization, ranging from $23.7 \%$ for non-disabled, heterosexual males, to $75 \%$ for disabled LGBQ males. Cell sizes ranged from 1718 (non-disabled heterosexual males not reporting peer victimization) to 16 (disabled GBQ males not reporting peer victimization) (Table 15). Among the intersectional categories with three statues combined, students most likely to report being victimized in the past 30 days were disabled GBQ males.

Design-based tests of independence (reporting the Rao-Scott correction F statistic instead of the Pearson $X^{2}$ ) revealed significant differences in exposure to peer victimization by each key independent variable (including the intersectional variables) (Table 15). Significant differences also existed among the eight regions $[F(3.55,241.43)$ $=2.592, \mathrm{p}=.0438]$, with the percentage of exposure to peer victimization ranging from $25.1 \%$ in one region, to $34.1 \%$ in another region.

The mean for the MHI-5 score (71.5) indicated, on the whole, positive mental health among respondents (Table 16). Generally, males and students without disabilities scored better on the MHI-5 items, compared to females and disabled students. Significant differences by disability status were found by MHI-5 total score (lower among disabled 
students), peer victimization, sex, race/ethnicity, and sexual orientation status. Students who were male $(t=19.44, \mathrm{p}<.000)$ and students who were non-disabled $(t=11.73, \mathrm{p}<$ .000) had significantly higher MHI scores than students who were female and students who were disabled; both of the latter groups were also more likely to report peer victimization $(33 \%, 39.9 \%$ respectively). Within the intersectional variable of disability and sex combined, disabled females had the lowest mean MHI score (62.4), and were more likely to report peer victimization (42.5\%). Non-disabled males had significantly higher mean MHI score (77.9) compared to all other groups, and were less likely to report peer victimization (25.3\%). 
Table 15

Characteristics of 11th Graders in Oregon (2008) Reporting Peer Victimization

\begin{tabular}{|c|c|c|c|c|c|c|}
\hline \multirow[b]{2}{*}{ Social status } & \multirow{2}{*}{$\begin{array}{c}\text { Valid } \\
n\end{array}$} & \multicolumn{2}{|c|}{ Not victimized } & \multicolumn{2}{|c|}{ Victimized } & \multirow[t]{2}{*}{$F^{\mathrm{a}}$} \\
\hline & & $n$ & $\%$ & $n$ & $\%$ & \\
\hline Total & 6718 & 4670 & 69.4 & 2048 & 30.6 & \\
\hline \multicolumn{7}{|l|}{ Sex } \\
\hline Male & 3198 & 2320 & 72.1 & 878 & 27.9 & $F(1,68)=$ \\
\hline Female & 3520 & 2350 & 66.99 & 1170 & 33 & $15.419 * * *$ \\
\hline Total & 6718 & 4670 & 69.42 & 2048 & 30.6 & \\
\hline \multicolumn{7}{|l|}{ Disability } \\
\hline Non-disabled & 5079 & 3697 & 72.4 & 1382 & 27.6 & $F(1,68)=$ \\
\hline Disabled & 1620 & 962 & 60.1 & 658 & 39.9 & $50.997 * * *$ \\
\hline Total & 6699 & 4659 & 69.5 & 2040 & 30.5 & \\
\hline \multicolumn{7}{|l|}{ Race/ethnicity } \\
\hline White & 4908 & 3492 & 71.5 & 1416 & 28.5 & $F(1,68)=$ \\
\hline Student of color $(\mathrm{SoC})^{\mathrm{b}}$ & 1695 & 1104 & 64 & 591 & 36 & $17.784 * * *$ \\
\hline Total & 6603 & 4596 & 69.4 & 2007 & 30.6 & \\
\hline \multicolumn{7}{|l|}{ Sexual orientation } \\
\hline Heterosexual $^{\mathrm{c}}$ & 5714 & 4122 & 71.7 & 1592 & 28.3 & $F(1,68)=$ \\
\hline $\mathrm{LGBQ}^{\mathrm{d}}$ & 443 & 199 & 49.4 & 244 & 50.6 & $48.233 * * *$ \\
\hline Total & 6157 & 4321 & 70 & 1836 & 30 & \\
\hline \multicolumn{7}{|l|}{ Disability and sex } \\
\hline Non-disabled, male & 2481 & 1877 & 74.7 & 604 & 25.3 & $F(3,66)=$ \\
\hline Non-disabled, female & 2598 & 1820 & 70.2 & 778 & 29.8 & $20.779 * * *$ \\
\hline Disabled, male & 708 & 438 & 63.4 & 270 & 36.6 & \\
\hline Disabled, female & 912 & 524 & 57.5 & 388 & 42.5 & \\
\hline Total & 6699 & 4659 & 69.5 & 2040 & 30.5 & \\
\hline \multicolumn{7}{|l|}{ Disability and race/ethnicity } \\
\hline Non-disabled, white & 3663 & 2724 & 74.4 & 939 & 25.6 & $F(3,66)=$ \\
\hline Non-disabled, SoC & 1332 & 915 & 67.3 & 417 & 32.7 & $17.522 * * *$ \\
\hline Disabled, white & 1240 & 765 & 62.7 & 475 & 37.3 & \\
\hline Disabled, student, SoC & 351 & 182 & 51 & 169 & 49 & \\
\hline Total & 6586 & 4586 & 69.4 & 2000 & 30.6 & \\
\hline \multicolumn{7}{|l|}{ Disability and sexual orientation } \\
\hline Non-disabled, heterosexual & 4411 & 3292 & 73.7 & 1119 & 26.3 & $F(3,66)=$ \\
\hline Disabled, heterosexual & 1290 & 822 & 64.8 & 468 & 35.2 & $21.713 * * *$ \\
\hline Non-disabled, LGBQ & 274 & 137 & 55.2 & 137 & 44.8 & \\
\hline Disabled, LGBQ & 168 & 62 & 38.6 & 106 & 61.4 & \\
\hline Total & 6143 & 4313 & 70 & 1830 & 30 & \\
\hline
\end{tabular}




\begin{tabular}{|c|c|c|c|c|c|c|}
\hline \multirow[b]{2}{*}{ Social status } & \multirow{2}{*}{$\begin{array}{c}\text { Valid } \\
n\end{array}$} & \multicolumn{2}{|c|}{ Not victimized } & \multicolumn{2}{|c|}{ Victimized } & \multirow[t]{2}{*}{$F^{\mathrm{a}}$} \\
\hline & & $n$ & $\%$ & $n$ & $\%$ & \\
\hline \multicolumn{7}{|l|}{ Disability, race and sex } \\
\hline Non-disabled, white, male & 1765 & 1366 & 77.8 & 399 & 22.3 & $F(7,62)=$ \\
\hline Non-disabled, white, female & 1898 & 1358 & 71.3 & 540 & 28.7 & $9.457 * * *$ \\
\hline Non-disabled, SoC, male & 664 & 474 & 67.9 & 190 & 32.1 & \\
\hline Non-disabled, SoC, female & 668 & 441 & 66.7 & 227 & 33.3 & \\
\hline Disabled, white, male & 538 & 347 & 67.1 & 191 & 32.9 & \\
\hline Disabled, white, female & 702 & 418 & 59.4 & 284 & 40.6 & \\
\hline Disabled, SoC, male & 154 & 81 & 50.7 & 73 & 49.4 & \\
\hline Disabled, SoC, female & 197 & 101 & 51.3 & 96 & 48.7 & \\
\hline Total & 6586 & 4586 & 69.4 & 2000 & 30.6 & \\
\hline \multicolumn{7}{|l|}{ Disability, sex and sexual orientation } \\
\hline Non-disabled, heterosexual, male & 2220 & 1718 & 76.3 & 502 & 23.7 & $F(7,62)=$ \\
\hline Disabled, heterosexual, male & 580 & 390 & 69.4 & 190 & 30.6 & $10.245 * * *$ \\
\hline Non-disabled, heterosexual, female & 2191 & 1574 & 71 & 617 & 29 & \\
\hline Disabled, heterosexual, female & 710 & 432 & 61.1 & 278 & 38.9 & \\
\hline Non-disabled LBQ, female & 175 & 93 & 59.8 & 82 & 40.2 & \\
\hline Disabled LBQ, female & 111 & 46 & 44.4 & 65 & 55.6 & \\
\hline Non-disabled GBQ, male & 99 & 44 & 46.9 & 55 & 53.1 & \\
\hline Disabled GBQ, male & 57 & 16 & 25 & 41 & 75 & \\
\hline Total & 6143 & 4313 & 70 & 1830 & 30 & \\
\hline
\end{tabular}

${ }^{a}$ Significance results reported are based upon design-based $F$ test ${ }^{6}$ Racial categories: white excludes Hispanic/Latino youth who identified as white, students of color (SOC) included students who identified as Hispanic/Latino. ${ }^{c}$ Analyses excluded students who identified as heterosexual and reported same sex experience. ${ }^{\mathrm{d}} \mathrm{LGBQ}=$ lesbian, gay, bisexual, questioning (not sure).

$* * * \mathrm{p}<.001$. 
Table 16

Psychosocial Distress of 11th Graders Reporting Peer Victimization by Sex and Disability

\begin{tabular}{|c|c|c|c|c|c|c|}
\hline \multirow{2}{*}{ Social status } & \multirow{2}{*}{$n$} & \multirow{2}{*}{$M$} & \multirow{2}{*}{$S E$} & \multicolumn{2}{|c|}{$95 \% \mathrm{CI}$} & \multirow{2}{*}{$t^{\mathrm{a}}$} \\
\hline & & & & LL & UL & \\
\hline All & 6822 & 71.5 & 0.21 & 71.1 & 72.0 & \\
\hline Sex & 6822 & & & & & \\
\hline Male & 3,268 & 75.6 & 0.27 & 75.1 & 76.1 & $1044^{* * *}$ \\
\hline Female & 3,554 & 67.8 & 0.3 & 67.2 & 68.4 & 19.44 \\
\hline Disability & 6804 & & & & & \\
\hline Non-disabled & 5157 & 73.3 & 0.23 & 72.8 & 73.7 & $11.73^{* * *}$ \\
\hline Disabled & 1647 & 66 & 0.59 & 64.8 & 67.2 & \\
\hline \multicolumn{7}{|l|}{ Disability and sex } \\
\hline Non-disabled, male & 2526 & 77 & 0.29 & 76.5 & 77.6 & $\begin{array}{l}6.84 \mathrm{~b}^{* * *} \\
18.1^{\mathrm{c} * * *}\end{array}$ \\
\hline Non-disabled, female & 2631 & 69.6 & 0.27 & 69.1 & 70.2 & $22.15^{\mathrm{d} * * *}$ \\
\hline Disabled, male & 730 & 70.6 & 0.88 & 68.9 & 72.4 & $9.74^{\mathrm{e}^{* * *}}$ \\
\hline Disabled, female & 917 & 62.4 & 0.75 & 60.9 & 63.9 & $7.18^{\mathrm{f} * * *}$ \\
\hline
\end{tabular}

Note. $n=6822 . \mathrm{CI}=$ confidence intervals; $\mathrm{LL}=$ lower limit; $\mathrm{UL}=$ upper limit. Psychosocial distress is measured by a transformed MHI-5 score, scaled 0 to 100; higher score indicates better mental health. ${ }^{a} t$ statistics reported are derived from design-based estimates (reported as $t$ ). ${ }^{b}$ Non-disabled males and disabled males. ${ }^{\mathrm{c}}$ Non-disabled males and disabled females. ${ }^{\mathrm{d}}$ Non-disabled males and females. ${ }^{\mathrm{e}}$ Disabled and non-disabled females. ${ }^{\mathrm{f}}$ Disabled males and females.

$* * * \mathrm{p}<.001$. 


\section{Part 1: Analyses (research questions 1.1 to 2.3)}

Of the 7091 respondents in the dataset, 6699 answered the questions for peer victimization, disability and sex. The sample size was reduced further to 6586 after excluding those missing on race/ethnicity. The final sample size for respondents answering questions related to peer victimization, disability, sex, sexual orientation, and race/ethnicity was 6045 . Results for each model are described below. The adjusted odd ratios are reported; all models, with the exception of the sixth model, controlled for region and other social status variables not directly being tested in the logistic regression, unless otherwise indicated. As an aid in following the findings in the context research questions and hypotheses associated with the first two aims, see Table 17.

Research question 1.1. ("Is disability status associated with peer victimization, and if so, to what extent?"). Results from logistic testing supported the hypothesis that students with disabilities were significantly more likely to report peer victimization compared to their non-disabled peers $(O R=1.7[1.57,1.93], \mathrm{p}<.001)$. After controlling for other demographic characteristics, sex, sexual orientation, and race/ethnicity were also significant predictors of exposure to peer victimization (Table 18, Model 1). Sexual orientation status stood out as a particularly important variable with relatively higher odds ratio reported for LGBQ youth compared to all other social statuses $(O R=2.4[1.97$, 2.92], $\mathrm{p}<.001)$.

Research question 2.1. ("Taking an intersectional approach, what is the relationship between (a) disability status with each of the three demographic variables sex, sexual orientation, and race/ethnicity, and (b) peer victimization, controlling for 
other demographic variables?"). This question involved conducting three logistic regressions, each with one of the three intersectional predictors: disability and sex, disability and race/ethnicity, and disability and sexual orientation. Results from Model 2, 3 , and 4 (Table 18) supported the hypothesis that the relationship between disability status and peer victimization changes significantly with the addition of a single variable, whether it be sex, race/ethnicity, or sexual orientation.

Logistic regression conducted with the intersectional variable of disability and sex supported my hypothesis that disabled students, compared to non-disabled students, would be more likely to report peer victimization (Table 18, Model 2). Additionally, not only were disabled youth more likely to report peer victimization, but there were also significant differences by sex as disabled females $(O R=2.0[1.74,2.35])$ were more likely to report exposure to peer victimization compared to non-disabled females $(O R=$ $1.3[1.13,1.44])$. Testing the equality of coefficients, using an adjusted Wald test, for disabled females and non-disabled females, indicated significant differences $(F(1,68)=$ $54.24, \mathrm{p}<.000)$.

Findings from the logistic regression containing the intersectional predictor of disability status and race/ethnicity confirmed that students with disabilities of any race/ethnicity would be more likely to report peer victimization, and disabled students of color were most likely to report exposure to peer victimization compared to their peers $(O R=2.4[1.97,3.02])($ Table 18, Model 3). However, the results did not align with my hypothesis that non-disabled students of color would be least likely to report peer victimization. Rather, disabled white students and disabled students of color, were both 
more likely to report peer victimization, compared to white non- disabled students $(O R=$ $1.4[1.21,1.64], O R=2.4[1.97,3.03]$ respectively).

Findings from logistic regression conducted with the intersectional variable of disability and sexual orientation also supported my hypothesis that the greatest exposure to peer victimization reported would be found among LGBQ students with disabilities $(O R=4.3[3.27,5.65])$, and that the lowest rates of exposure to peer victimization would be found among non-disabled males identifying as heterosexual and not reporting same sex experience (reference group) (Table 18, Model 4).

Research question 2.2. ("Taking an intersectional approach, what is the relationship between (a) disability status with sex, and racelethnicity, and (b) peer victimization, controlling for other demographic variables?"). My hypothesis that disability status would be the strongest influence on exposure to peer victimization, followed by racial/ethnic status, was partially supported. However, my hypothesis that students of color would be represented at both ends of the spectrum, with male nondisabled students of color least likely, and female students of color and/or Latino with disabilities most likely, to report peer victimization, was not supported (Table 18, Model $5)$.

The odd ratios reported in Table 18 (Model 5) reveal the relatively close distance between all seven subgroups (excluding the referent group). While significant differences were found between the subgroups at both ends of spectrum (e.g., differences between non-disabled white females compared to disabled males of color $[F(1,68)=20.91, \mathrm{p}<$ $.001]$, there were a number of subgroups that did not differ (Table 19). Several subgroups 
which differed only by sex were not found to be significantly different from each other; significant differences were not detected between non-disabled males and females of color, nor between disabled white males and females. Other non-significant differences in exposure to peer victimization were found between: (1) non-disabled white females and non-disabled males of color, (2) white disabled males and white non-disabled females, and (3) disabled white males and non-disabled females of color.

Research question 2.3. ("Taking an intersectional approach, what is the relationship between (a) disability status with sex, and sexual orientation, and (b) peer victimization, controlling for other demographic variables?"). As hypothesized, male GBQ students with disabilities were most likely to report peer victimization $(O R=10.8$ $[5.84,19.9])$, while male students without disabilities who identified as heterosexual and did not report having same sex experience, as the referent group, were least likely to report peer victimization (Table 18, Model 6). Sex was also a strong predictor when combined with sexual orientation status, as seen in the relatively lower odds of LBQ females reporting peer victimization compared to their GBQ male peers, regardless of disability status. Caution is needed, however, due to instability in cell sizes as the standard error was considerable $(S E=3.31)$ in regards to the odds for disabled GBQ males, with larger variability in the $95 \%$ confidence intervals.

My hypothesis that sex and sexual orientation combined with disability status would be stronger predictors of exposure to peer victimization than disability status alone was supported, as seen in the lower odds for disabled heterosexual males $(O R=1.5[1.27$, $1.79])$ and females $(O R=2.2[1.81,2.56])$, compared to those of students who identified 
as LGBQ, particularly for non-disabled GBQ males $(O R=3.5[2.26,5.55])$, disabled LBQ females $(O R=4.0[3.06,5.25)]$ and disabled GBQ males $(O R=10.8[5.84,19.9])$ (Table 18, Model 6).

While all sub-groups reported significantly greater odds than the referent group (non-disabled heterosexual males), there were a few cases in which non-significant differences were detected between groups (Table 20). A visual glance of the odd ratios reported in Table 18 (Model 6) provides a clue to which subgroups would likely be significantly different from each other; however there were a number of subgroups that did not differ. Specifically, differences were not detected between: non-disabled LBQ females and non-disabled GBQ males (sex differences), disabled heterosexual females and non-disabled heterosexual females (disability differences), disabled LBQ females and non-disabled GBQ males (disability and sex combined differences), and disabled heterosexual females and non-disabled LBQ females (disability and sexual orientation combined differences).

To summarize, results of logistic tests (Table 18) suggest that those who are disabled have the highest odds ratios alone and in combination with other statuses. Further, the relationship between disability status and peer victimization does change, and the magnitude of change varies by specific intersectional statuses of the students. For example, while disabled students were most likely to report exposure to peer victimization, compared to non-disabled students, the odds of exposure to peer victimization among disabled females was greater than the odds reported for disabled students alone. 
The findings from the fourth logistic model provided the sharpest contrast on the effects of disability and one single status (sexual orientation) combined. The odds ratio of disabled LGBQ students $(O R=4.3[3.27,5.65])$, with non-disabled heterosexual students as the referent group, was considerably larger than that of: (1) disabled students, compared to non-disabled students $(O R=1.6[1.44,1.77])$, and (2) LGBQ students $(O R=$ $2.4[1.97,2.92])$ compared to heterosexual students (reporting no same sex experience).

The relative magnitude of these odds grew considerably with the insertion of a third variable, as seen in Model 5 and 6 (Table 18). It is also important to consider the statistical significant differences between subgroups as shown in Table 19 and Table 20.

Differences in the odds of reporting exposure to peer victimization among disabled students, by sex and race/ethnicity, reveal the relative differences within groups in exposure to peer victimization. While disabled white males were significantly more likely to report exposure to peer victimization compared to non-disabled white males, they were significantly less likely to report peer victimization compared to other disabled students who differed by sex or racial status. Similarly, while disabled heterosexual males were more likely to report exposure to peer victimization compared to non-disabled heterosexual males, they were far less likely to report peer victimization compared to LGBQ students, with and without disabilities. 
Table 17

Summary of Findings in Context of Aims $1 \& 2$, and Associated Research Questions and Hypotheses

\begin{tabular}{|c|c|c|c|}
\hline Study Aims & Research Questions & Hypotheses & Findings \\
\hline $\begin{array}{l}\text { AIM 1: To } \\
\text { examine the } \\
\text { extent to which } \\
\text { disability status } \\
\text { is associated } \\
\text { with exposure to } \\
\text { PV. }\end{array}$ & $\begin{array}{l}\text { 1.1. Is disability } \\
\text { status associated with } \\
\text { PV, and if so, to what } \\
\text { extent? }\end{array}$ & $\begin{array}{l}\text { 1.1.1. Disability and PV. } \\
\text { Most likely: Students with } \\
\text { disabilities }\end{array}$ & $\begin{array}{l}\text { Most likely: Supported } \\
\text { OR }=1.6[1.44,1.77] . \\
\text { (Model 1, Table 18) }\end{array}$ \\
\hline \multirow{4}{*}{$\begin{array}{l}\text { AIM 2: Taking } \\
\text { an intersectional } \\
\text { approach, } \\
\text { investigate the } \\
\text { extent to which } \\
\text { disability status } \\
\text { in combination } \\
\text { with two sets of } \\
\text { identity variables } \\
\text { (sexual } \\
\text { orientation, sex; } \\
\text { and race/ } \\
\text { ethnicity, sex) is } \\
\text { associated with } \\
\text { exposure to PV. }\end{array}$} & \multirow{3}{*}{$\begin{array}{l}\text { 2.1. Taking an } \\
\text { intersectional } \\
\text { approach, what is the } \\
\text { relationship between } \\
\text { (a) disability status } \\
\text { with each of the } \\
\text { three demographic } \\
\text { variables - sex, } \\
\text { sexual orientation, } \\
\text { and race/ethnicity, } \\
\text { and (b) PV, } \\
\text { controlling for other } \\
\text { demographic } \\
\text { variables? }\end{array}$} & $\begin{array}{l}\text { 2.1.1. Disability and sex. } \\
\text { Most likely: Disabled } \\
\text { females; Least likely: Non- } \\
\text { disabled males }\end{array}$ & $\begin{array}{l}\text { Most likely: Supported } \\
\text { OR }=2[1.74,2.35] . \\
\text { Least likely: Supported } \\
\text { OR }=1 \text { (reference group). } \\
\text { (Model 2, Table 18) }\end{array}$ \\
\hline & & $\begin{array}{l}\text { 2.1.2. Disability and race/ } \\
\text { ethnicity. Most likely: } \\
\text { Disabled students of color; } \\
\text { Least likely: Non-disabled } \\
\text { students of color }\end{array}$ & $\begin{array}{l}\text { Most likely: Supported } \\
\text { OR }=2.4[1.97,3.03] . \\
\text { Least likely: Not } \\
\text { supported. } \\
\text { (Model 3, Table 18) }\end{array}$ \\
\hline & & $\begin{array}{l}\text { 2.1.3. Disability and } \\
\text { sexual orientation. } \\
\text { Most likely: Disabled } \\
\text { LGBQ; Least likely: Non- } \\
\text { disabled heterosexual }\end{array}$ & $\begin{array}{l}\text { Most likely: Supported } \\
\text { OR }=4.3[3.27,5.65] . \\
\text { Least likely: Supported } \\
\text { OR }=1 \text { (reference group) } \\
\text { (Model 4, Table 18) }\end{array}$ \\
\hline & $\begin{array}{l}\text { 2.2. Taking an } \\
\text { intersectional } \\
\text { approach, what is the } \\
\text { relationship between } \\
\text { (a) disability status } \\
\text { with sex, and } \\
\text { race/ethnicity, and } \\
\text { (b) PV, controlling } \\
\text { for other } \\
\text { demographic } \\
\text { variables? }\end{array}$ & $\begin{array}{l}\text { Most likely: Disabled } \\
\text { female students of color. } \\
\text { Least likely: Non-disabled } \\
\text { male students of color }\end{array}$ & $\begin{array}{l}\text { Most likely: Partially } \\
\text { supported; disabled } \\
\text { female students of color } \\
(O R=2.95[2.24,3.89] \\
\text { almost as likely as disabled } \\
\text { male students of color (OR } \\
=2.96[2.14,4.10] . \\
\text { Least likely: Not } \\
\text { supported. (Model } 5 \text {, } \\
\text { Table 18) }\end{array}$ \\
\hline
\end{tabular}

\begin{tabular}{|c|c|c|}
\hline $\begin{array}{l}\text { 2.3. Taking an } \\
\text { intersectional } \\
\text { approach, what is the } \\
\text { relationship between } \\
\text { (a) disability status } \\
\text { with sex, and sexual } \\
\text { orientation, and (b) } \\
\text { PV, controlling for } \\
\text { other demographic } \\
\text { variables? }\end{array}$ & $\begin{array}{l}\text { 2.3.1. Disability, sex, sex } \\
\text { orientation and peer } \\
\text { victimization. } \\
\text { Most likely: Male GBQ } \\
\text { students with disabilities } \\
\text { Least likely: Non-disabled } \\
\text { heterosexual male students }\end{array}$ & $\begin{array}{l}\text { Most likely: Supported } \\
\text { OR }=10.8 \text { [5.84. 19.9]. } \\
\text { Least likely: Supported } \\
\text { OR }=1 \text { (reference group) } \\
\text { (Model 6, Table 18) }\end{array}$ \\
\hline
\end{tabular}

Note. $\mathrm{PV}=$ peer victimization. $O R=$ adjusted odds ratio with peer victimization as dependent variable. Brackets contain the lower and upper limits of the $95 \%$ confidence interval. 
Table 18

Adjusted Odds of Reporting Peer Victimization

\begin{tabular}{|c|c|c|c|c|c|c|}
\hline \multirow{2}{*}{ Predictor (referent group) } & \multirow{2}{*}{$A O R$} & \multirow{2}{*}{$S E$} & & \multirow{2}{*}{$p$} & \multicolumn{2}{|c|}{$95 \% \mathrm{CI}$} \\
\hline & & & & & LL & UL \\
\hline \multicolumn{7}{|l|}{ Model 1} \\
\hline Constant & 0.32 & 0.02 & -15.14 & 0.000 & 0.27 & 0.37 \\
\hline Region ID & 0.97 & 0.01 & -2.53 & 0.014 & 0.94 & 0.99 \\
\hline Sex (males) & 1.27 & 0.08 & 4.06 & 0.000 & 1.13 & 1.43 \\
\hline Race/ethnicity (white) ${ }^{\mathrm{a}}$ & 1.45 & 0.09 & 5.99 & 0.000 & 1.28 & 1.64 \\
\hline Disability status (non-disabled) & 1.6 & 0.08 & 9.16 & 0.000 & 1.44 & 1.77 \\
\hline Sexual orientation (heterosexual) ${ }^{\mathrm{b}}$ & 2.4 & 0.24 & 8.85 & 0.000 & 1.97 & 2.92 \\
\hline \multicolumn{7}{|c|}{ Model $F(5,64)=47.12, \mathrm{p}<.000 . \quad F$-adjusted goodness of fitness test $=F(9,60)=0.764, \mathrm{p}=.649$} \\
\hline \multicolumn{7}{|l|}{ Model 2 - Disability and Sex } \\
\hline Constant & 0.32 & 0.02 & -15.85 & 0.000 & 0.27 & 0.37 \\
\hline Region ID & 0.97 & 0.01 & -2.53 & 0.014 & 0.94 & 0.99 \\
\hline Race/ethnicity (white) & 1.45 & 0.09 & 6.01 & 0.000 & 1.28 & 1.64 \\
\hline Sexual orientation (heterosexual) & 2.4 & 0.24 & 8.88 & 0.000 & 1.97 & 2.92 \\
\hline \multicolumn{7}{|c|}{ Disability and sex (non-disabled males) } \\
\hline Non-disabled females & 1.28 & 0.08 & 4.02 & 0.000 & 1.13 & 1.44 \\
\hline Disabled males & 1.61 & 0.12 & 6.17 & 0.000 & 1.38 & 1.87 \\
\hline Disabled females & 2.02 & 0.15 & 9.36 & 0.000 & 1.74 & 2.35 \\
\hline Model $F(6,63)=42.53, \mathrm{p}<.000 . F$-ad & ed gooc & $0 \mathrm{cof}$ & ecs t to & $=E(0) 6$ & 0 & $=.661$ \\
\hline \multicolumn{7}{|l|}{ Model 3 - Disability and race/ethnicity } \\
\hline Constant & 0.32 & 0.03 & -14.45 & 0.000 & 0.27 & 0.38 \\
\hline Region ID & 0.96 & 0.01 & -2.55 & 0.013 & 0.94 & 0.99 \\
\hline Sexual orientation (heterosexual) & 2.4 & 0.24 & 8.9 & 0.000 & 1.97 & 2.92 \\
\hline Sex (males) & 1.27 & 0.08 & 4.06 & 0.000 & 1.13 & 1.43 \\
\hline \multicolumn{7}{|c|}{ Disability and race/ethnicity ( non-disabled white, non-Hispanic students) } \\
\hline Non-disabled student of color & 1.41 & 0.11 & 4.55 & 0.000 & 1.21 & 1.64 \\
\hline Disabled white & 1.55 & 0.09 & 7.82 & 0.000 & 1.39 & 1.73 \\
\hline Disabled student of color & 2.44 & 0.26 & 8.36 & 0.000 & 1.97 & 3.03 \\
\hline Model $F(6,63)=38.53, \mathrm{p}<.000 . F$-ad & d & f & moto & $=F(9$, & & $=.518$ \\
\hline \multicolumn{7}{|l|}{ Model 4 - Disability and sexual orientation } \\
\hline Constant & 0.32 & 0.02 & -14.79 & 0.000 & 0.27 & 0.37 \\
\hline Region ID & 0.97 & 0.01 & -2.54 & 0.014 & 0.94 & 0.99 \\
\hline Race/ethnicity (white) & 1.45 & 0.09 & 6.05 & 0.000 & 1.28 & 1.64 \\
\hline Sex (males) & 1.27 & 0.08 & 4.07 & 0.000 & 1.13 & 1.43 \\
\hline
\end{tabular}




\begin{tabular}{|c|c|c|c|c|c|c|}
\hline \multirow{2}{*}{ Predictor (referent group) } & \multirow{2}{*}{$A O R$} & \multirow{2}{*}{$S E$} & & \multirow{2}{*}{$p$} & \multicolumn{2}{|c|}{$95 \% \mathrm{CI}$} \\
\hline & & & & & LL & UL \\
\hline \multicolumn{7}{|c|}{ Disability and sexual orientation (non-disabled heterosexual) } \\
\hline Disabled heterosexual & 1.56 & 0.08 & 8.57 & 0.000 & 1.41 & 1.73 \\
\hline Non-disabled LGBQ & 2.24 & 0.29 & 6.33 & 0.000 & 1.74 & 2.89 \\
\hline \multicolumn{7}{|c|}{$\begin{array}{l}\text { Model } F(6,63)=39.63, \mathrm{p}<.000 . F \text {-adjusted goodness of fitness test }=F(9,60)=0.769, \mathrm{p}= \\
.645\end{array}$} \\
\hline \multicolumn{7}{|l|}{ Model 5 - Disability, sex, and race/ethnicity } \\
\hline Constant & 0.27 & 0.02 & -21.4 & 0.000 & 0.24 & 0.30 \\
\hline Sexual orientation (heterosexual) & 2.40 & 0.23 & 9.12 & 0.000 & 1.98 & 2.91 \\
\hline \multicolumn{7}{|c|}{ Disability, sex, and race/ethnicity (non-disabled white, non-Hispanic males) } \\
\hline Non-disabled white females & 1.39 & 0.09 & 4.94 & 0.000 & 1.22 & 1.59 \\
\hline Disabled white males & 1.56 & 0.14 & 5.07 & 0.000 & 1.31 & 1.85 \\
\hline Non-disabled males of color & 1.65 & & .7 & & 1.34 & 05 \\
\hline Non-disabled females of color & 1.74 & 0.13 & 7.48 & 0.000 & 1.5 & 2.02 \\
\hline Disabled white females & 2.13 & 0.19 & 8.6 & 0.000 & 1.79 & 2.54 \\
\hline Disabled females of color & 2.95 & 0.41 & 7.84 & 0.000 & 2.24 & 3.89 \\
\hline Disabled males of color & & 0.48 & 6.7 & & & \\
\hline Model $F(8,61)=29.14, \mathrm{p}<.000 . \quad F$-adjl & & & & & & \\
\hline \multicolumn{7}{|c|}{ Model 6 - Disability, sex, and sexual orientation } \\
\hline Constant & 0.36 & 0.02 & -14.86 & 0.000 & 0.31 & 0.41 \\
\hline Region ID & 0.97 & 0.01 & -2.51 & 0.014 & 0.94 & 0.99 \\
\hline Race/ethnicity (white) & 1.44 & 0.09 & 5.91 & 0.000 & 1.27 & 1.63 \\
\hline \multicolumn{7}{|c|}{ Disability, sex, and sexual orientation (non-disabled heterosexual males) } \\
\hline Non-disabled heterosexual females & 1.35 & 0.08 & 5.04 & 0.000 & 1.2 & 1.52 \\
\hline Disabled heterosexual males & 1.5 & 0.13 & 4.74 & 0.000 & 1.27 & 1.79 \\
\hline Disabled heterosexual females & 2.15 & 0.19 & 8.92 & 0.000 & 1.81 & 2.56 \\
\hline Non-disabled LBQ females & 2.3 & 0.34 & 5.69 & 0.000 & 1.72 & 3.08 \\
\hline Non-disabled GBQ males & 3.54 & 0.8 & 5.6 & 0.000 & 2.26 & 5.55 \\
\hline Disabled LBQ females & 4.0 & 0.54 & 10.24 & 0.000 & 3.06 & 5.25 \\
\hline \multicolumn{7}{|c|}{$\begin{array}{l}\text { Model } F(9,60)=31.32, \mathrm{p}<.000 . F \text {-adjusted goodness of fitness test }=F(9,60)=2.118, \mathrm{p}= \\
.042\end{array}$} \\
\hline \multicolumn{7}{|c|}{$\begin{array}{l}\text { Note. } N=6045 . A O R=\text { adjusted odds ratio with peer victimization as dependent variable. } \\
\text { Subgroups are in order of magnitude of AORs. CI = confidence interval; } \mathrm{LL}=\text { lower limit; } \mathrm{UL}= \\
\text { upper limit. Significance results reported are based upon survey design-based tests. } \\
\text { a Racial categories: white excluded youth identifying as white and Hispanic/Latino youth; students } \\
\text { of color included students who identified as Hispanic/Latino. }{ }^{b} \text { Analyses excluded students who } \\
\text { identified as heterosexual and reported same sex experience. }\end{array}$} \\
\hline
\end{tabular}


Table 19

Tests of Independence for Exposure to Peer Victimization by Disability, Sex, and Race/ethnicity

\begin{tabular}{|c|c|c|c|}
\hline & & $F(1,68)$ & $\mathrm{p}$ value \\
\hline \multicolumn{4}{|c|}{ Differences by disability } \\
\hline DWm (4) & $=\operatorname{NdWm}(0)$ & 25.74 & $* * *$ \\
\hline $\operatorname{DWf}(5)$ & $=\operatorname{NdWf}(1)$ & 39.29 & $* * *$ \\
\hline DNwm (6) & $=\operatorname{NdNwm}(2)$ & 10.65 & $* *$ \\
\hline DNwf (7) & $=\operatorname{NdNwf}(3)$ & 13.84 & $* * *$ \\
\hline \multicolumn{4}{|c|}{ Differences by sex } \\
\hline $\operatorname{NdWm}(0)$ & $=\operatorname{NdWf}(1)$ & 24.38 & $* * *$ \\
\hline NdNwm (2) & $=\operatorname{NdNwf}(3)$ & 0.19 & \\
\hline DWm (4) & $=\operatorname{DWf}(5)$ & 6.63 & $*$ \\
\hline DNwm (6) & $=\operatorname{DNwf}(7)$ & 0.00 & \\
\hline \multicolumn{4}{|c|}{ Differences by race } \\
\hline $\operatorname{NdWm}(0)=$ & $=\operatorname{NdNwm}(2)$ & 22.1 & $* * *$ \\
\hline $\operatorname{NdWf}(1)=$ & $=\operatorname{NdNwf}(3)$ & 6.34 & $*$ \\
\hline $\operatorname{DWm}(4)=$ & $=\operatorname{DNwm}(6)$ & 12.08 & $* * *$ \\
\hline $\operatorname{DWf}(5)=$ & $=\operatorname{DNwf}(7)$ & 5.78 & $*$ \\
\hline \multicolumn{4}{|c|}{ Differences by race and sex } \\
\hline $\operatorname{NdWm}(0)=$ & $=\operatorname{NdNwf}(3)$ & 55.91 & $* * *$ \\
\hline $\operatorname{NdWf}(1)=$ & $=\operatorname{NdNwm}(2)$ & 2.05 & \\
\hline $\operatorname{DWm}(4)=$ & $=\operatorname{DNwf}(7)$ & 18.72 & $* * *$ \\
\hline $\operatorname{DWf}(5)=$ & $=$ DNwm (6) & 4.11 & $*$ \\
\hline \multicolumn{4}{|c|}{ Differences by disability and sex } \\
\hline DWf (5) & $=\operatorname{NdWm}(0)$ & 73.94 & $* * *$ \\
\hline $\operatorname{DWm}(4)=$ & $=\operatorname{NdWf}(1)$ & 1.04 & \\
\hline $\operatorname{DNwf}(7)=$ & $=\operatorname{NdNwm}(2)$ & 14.25 & $* * *$ \\
\hline $\operatorname{DNwm}(6)=$ & $=\operatorname{MdNwf}(3)$ & 9.50 & $* *$ \\
\hline \multicolumn{4}{|c|}{ Differences by disability and race/ethnicity } \\
\hline $\operatorname{DNwm}(6)=$ & $=\operatorname{NdWm}(0)$ & 44.86 & $* * *$ \\
\hline $\operatorname{DNwf}(7)=$ & $=\operatorname{NdWf}(1)$ & 38.17 & $* * *$ \\
\hline $\operatorname{DWm}(4)=$ & $=\operatorname{NdNwm}(2)$ & 0.22 & \\
\hline $\operatorname{DWf}(5)=$ & $=\operatorname{NdNwf(3)}$ & 4.08 & $*$ \\
\hline \multicolumn{4}{|c|}{ Differences by disability, race/ethnicity and sex } \\
\hline $\operatorname{DNwf}(7)=$ & $=\operatorname{NdWm}(0)$ & 61.51 & $* * *$ \\
\hline $\operatorname{DNwm}(6)=$ & $=\operatorname{NdWf}(1)$ & 20.91 & $* * *$ \\
\hline $\operatorname{DWf}(5)=$ & $=\operatorname{NdNwm}(2)$ & 6.06 & $*$ \\
\hline $\operatorname{DWm}(4)=$ & $=\operatorname{NdNwf}(3)$ & 0.95 & \\
\hline
\end{tabular}

Note. Differences in coefficients based upon logistic results (see Table 18, Model 5). The number within the parenthesis indicates number of the subgroup assigned. Significance results reported are based upon survey design-based tests. $\mathrm{D}=$ disabled, $\mathrm{Nd}=$ non-disabled, $\mathrm{W}=$ white, nonHispanic, $\mathrm{Nw}=$ non-white, non-Hispanic, $\mathrm{f}=$ female, $\mathrm{m}=$ male.

$* * * p<.001, * * p<.01, * p<.05$ 
Table 20

Tests of Independence for Peer Victimization by Disability, Sex, and Sexual Orientation

\begin{tabular}{|c|c|c|c|}
\hline & & $F(1,68)$ & $p$ value \\
\hline \multicolumn{4}{|c|}{ Differences by disability } \\
\hline $\operatorname{DHm}(1)$ & $=\operatorname{NdHm}(0)$ & 22.45 & $* * *$ \\
\hline $\operatorname{DHf}(3)$ & $=\operatorname{NdHf}(2)$ & 54.8 & $* * *$ \\
\hline DQf (5) & $=\operatorname{NdQf}(4)$ & 7.14 & $* *$ \\
\hline DQm (7) & $=\mathrm{NdQm}(6)$ & 11.05 & $* *$ \\
\hline \multicolumn{4}{|c|}{ Differences by sex } \\
\hline $\operatorname{DHf}(3)$ & $=\operatorname{DHm}(1)$ & 8.94 & $* *$ \\
\hline $\mathrm{NdHm}(0)$ & $=\operatorname{NdHf}(2)$ & 25.39 & $* * *$ \\
\hline NdQf (4) & $=\operatorname{NdQm}(6)$ & 3.14 & \\
\hline DQf $(5)$ & $=\mathrm{DQm}(7)$ & 7.86 & $* *$ \\
\hline \multicolumn{4}{|c|}{ Differences by sexual orientation } \\
\hline NdHM (0) & $=\operatorname{NdQM}(6)$ & 31.37 & $* * *$ \\
\hline DHm (1) & $=\operatorname{DQm}(7)$ & 31.89 & $* * *$ \\
\hline $\operatorname{NdHf}(2)$ & $=\operatorname{NdQf}(4)$ & 15.25 & $* * *$ \\
\hline $\mathrm{DHf}(3)$ & $=\operatorname{DQf}(5)$ & 16.43 & $* * *$ \\
\hline \multicolumn{4}{|c|}{ Differences by disability and sex } \\
\hline $\mathrm{DHm}(1)$ & $=\operatorname{NdHf}(2)$ & 1.08 & \\
\hline $\operatorname{DHf}(3)$ & $=\mathrm{NdHm}(0)$ & 79.58 & $* * *$ \\
\hline DQf (5) & $=\mathrm{NdQm}(6)$ & 0.21 & \\
\hline $\operatorname{DQm}(7)$ & $=\operatorname{NdQf}(4)$ & 21.43 & $* * *$ \\
\hline \multicolumn{4}{|c|}{ Differences by sex and sexual orientation } \\
\hline $\operatorname{NdHM}(0)$ & $=\operatorname{NdQf}(4)$ & 32.37 & $* * *$ \\
\hline $\mathrm{DHm}(1)$ & $=\operatorname{DQf}(5)$ & 47.7 & $* * *$ \\
\hline $\operatorname{NdHf}(2)$ & $=\operatorname{NdQm}(6)$ & 17.4 & $* * *$ \\
\hline DHf (3) & $=\operatorname{DQm}(7)$ & 28.59 & $* * *$ \\
\hline \multicolumn{4}{|c|}{ Differences by disability and sexual orientation } \\
\hline $\mathrm{DHm}(1)$ & $=\operatorname{NdQm}(6)$ & 11.1 & $* *$ \\
\hline DHf (3) & $=\operatorname{NdQf}(4)$ & 0.2 & \\
\hline $\operatorname{DQf}(5)$ & $=\operatorname{NdHf}(2)$ & 52.76 & $* * *$ \\
\hline $\mathrm{DQm}(7)$ & $=\mathrm{NdHm}(0)$ & 59.87 & $* * *$ \\
\hline \multicolumn{4}{|c|}{ Differences by disability, sexual orientation and sex } \\
\hline $\mathrm{DHm}(1)$ & $=\operatorname{NdQf}(4)$ & 6.2 & * \\
\hline DHf (3) & $=\mathrm{NdQm}(6)$ & 5.09 & $*$ \\
\hline $\operatorname{DQf}(5)$ & $=\mathrm{NdHm}(0)$ & 104.94 & $* * *$ \\
\hline $\operatorname{DQm}(7)$ & $=\operatorname{NdHf}(2)$ & 45.38 & $* * *$ \\
\hline
\end{tabular}

Note. Differences in coefficients based upon logistic results (see Table 18, Model 6). The number within the parenthesis indicates number of the subgroup assigned. Significance results reported are based upon survey design-based tests. $\mathrm{D}=$ disabled, $\mathrm{H}=$ heterosexual (and no same sex contact), $\mathrm{m}=$ male, $\mathrm{Nd}=$ non-disabled, $\mathrm{Q}=$ lesbian, gay, bisexual or questioning, $\mathrm{f}=$ female . $* * * p<.001, * * p<.01, * p<.05$ 


\section{Part 2: Analyses (Research Question 3.1 to 3.3)}

Of the 7091 respondents in the dataset, 6664 students answered survey questions related to disability status, peer victimization, and psychosocial distress (at least four of the five MHI items). As an aid in following the findings in the context of the research questions and hypotheses associated with the third aim, see Table 21.

Research question 3.1. ("Is disability status associated with psychosocial distress, and if so, to what extent?"). Results from the linear regression of psychosocial distress on disability status supported the hypothesis that students with disabilities are more likely to report symptoms of psychosocial distress compared to their non-disabled peers. The total effect (path c) of disability status on psychosocial distress was significant for both males and females; mental health scores (MHI-5) were significantly lower for female and males students with disabilities by seven and six points respectively, relative to female and male students without disabilities (Figure 3a).

Research question 3.2. ("Is the relationship between disability status and psychosocial distress mediated by exposure to peer victimization?"). Findings supported my hypothesis that exposure to peer victimization partially mediated the relationship between disability status and psychosocial distress (Figure 3b). The strength of the association between disability status and psychosocial distress decreased (path c'< path c) with the inclusion of exposure to peer victimization in the model. However, as the relationship between disability status and psychosocial distress remained significant (path c') for females and males (Figure 3b), partial mediation is indicated, rather than full mediation. The indirect effect, or the mediated effect (ab), was significant for both 
females $(-1.52[-1.9,-1.15])$ and males $(-1.15[-1.55,-0.76]))$. However, an examination of the confidence intervals in Table 22 and the differences between path c and c' suggests that for both females $\left(c^{-} c^{\prime}=1.47\right)$ and males $\left(c^{-} c^{\prime}=1.08\right)$ is close. The proportion of the mediated effects of exposure to peer victimization on the relationship between disability status and psychosocial distress was relatively small given by the measure of the proportion mediated (MacKinnon, 2008), which ranged from $17 \%$ to $21 \%$ for females, and $16 \%$ to $18 \%$ for males (Table 23 ).

Following mediation testing, I checked the normality of the MHI-5 total score measure following the linear regression, in order to determine the appropriateness of the model. The distributions of the MHI-5 total scores (ranged from 0 to100) were somewhat positively skewed, violating assumptions of normality. This is not unusual as the mean score for the U.S. general population using this measure was 75 , which is slightly higher than the mean for this study (71.5) (Ware et al., 2007). Efforts to transform this variable were not satisfactory; a decision was made to use the results involved in the analyses.

\section{Research question 3.3. ("Is the mediated effect of peer victimization on the} relationship between disability status and psychosocial distress moderated by sex?). While slight sex differences in the path coefficients were observed, particularly on path $b$, the difference only amounts to just over one point on the MHI-5 scale (scaled from 0 to 100) (Figure 3; Table 22). Nonetheless, post-hoc moderated mediation analyses were conducted as recommended by Edwards and Lambert (2007) which confirmed that sex did not significantly moderate the effects of peer victimization on psychosocial distress controlling for disability status (path b). 
Table 21

Summary of Findings in Context of Aim 3 and Associated Research Questions and Hypotheses

\begin{tabular}{|c|c|c|c|}
\hline Study Aims & $\begin{array}{l}\text { Research } \\
\text { Questions }\end{array}$ & Hypotheses & Findings \\
\hline \multirow{3}{*}{$\begin{array}{l}\text { AIM 3: To examine } \\
\text { the extent to which } \\
\text { disability status is } \\
\text { associated with } \\
\text { psychosocial } \\
\text { distress, and if so, } \\
\text { determine if the } \\
\text { effects of disability } \\
\text { status on } \\
\text { psychosocial } \\
\text { distress are } \\
\text { mediated by } \\
\text { exposure to peer } \\
\text { victimization. }\end{array}$} & $\begin{array}{l}\text { 3.1. Is disability } \\
\text { status associated } \\
\text { with psychosocial } \\
\text { distress, and if so, } \\
\text { to what extent? }\end{array}$ & $\begin{array}{l}\text { 3.1.1. Disability and } \\
\text { psychosocial distress. } \\
\text { Disability is } \\
\text { significantly associated } \\
\text { with PD }\end{array}$ & $\begin{array}{l}\text { Supported. The total effect } \\
\text { (path c) of disability status } \\
\text { on psychosocial distress was } \\
\text { significant for both males } \\
\text { and females. MHI-5 scores } \\
\text { were significantly lower for } \\
\text { female and males students } \\
\text { with disabilities by } 7 \text { and } 6 \\
\text { points respectively. }\end{array}$ \\
\hline & $\begin{array}{l}\text { 3.2. Is the } \\
\text { relationship } \\
\text { between disability } \\
\text { status and } \\
\text { psychosocial } \\
\text { distress mediated } \\
\text { by exposure to } \\
\text { peer } \\
\text { victimization? }\end{array}$ & $\begin{array}{l}\text { 3.2.1. Mediation: } \\
\text { disability, psychosocial } \\
\text { distress, and peer } \\
\text { victimization. } \\
\text { Exposure to peer } \\
\text { victimization partially } \\
\text { mediates the } \\
\text { relationship between } \\
\text { disability status and } \\
\text { psychosocial distress. }\end{array}$ & $\begin{array}{l}\text { Supported. Path c'< path c; } \\
\text { the indirect effect, or the } \\
\text { mediated effect }(\mathrm{ab}) \text {, was } \\
\text { significant for both females } \\
\text { and males. The proportion } \\
\text { mediated ranged from } 17 \% \\
\text { to } 21 \% \text { for females, and } \\
16 \% \text { to } 18 \% \text { for males } \\
\text { (Table } 24 \text { ) }\end{array}$ \\
\hline & $\begin{array}{l}\text { 3.3. Is the } \\
\text { mediated effect of } \\
\text { peer victimization } \\
\text { on the relationship } \\
\text { between disability } \\
\text { status and } \\
\text { psychosocial } \\
\text { distress } \\
\text { moderated by } \\
\text { sex? }\end{array}$ & $\begin{array}{l}\text { 3.3.1. Moderated } \\
\text { mediation: sex, } \\
\text { psychosocial distress, } \\
\text { and peer victimization. } \\
\text { The mediated effect of } \\
\text { peer victimization on } \\
\text { the relationship } \\
\text { between disability } \\
\text { status and psychosocial } \\
\text { distress is stronger for } \\
\text { females. }\end{array}$ & $\begin{array}{l}\text { Not supported. The } \\
\text { difference in the path } \\
\text { coefficients for both groups } \\
\text { (males and females) is just } \\
\text { over one point on the MHI- } \\
5 \text { scale. Post-hoc moderated } \\
\text { mediation analyses } \\
\text { confirmed that sex did not } \\
\text { significantly moderate the } \\
\text { effects of peer victimization } \\
\text { on psychosocial distress } \\
\text { controlling for disability } \\
\text { status (path b). }\end{array}$ \\
\hline
\end{tabular}

Note. Psychosocial distress is indicated by lower MHI5 total scores (scaled from 0 to 100). PV= peer victimization. 
$3 a$

c

Females: $-7.333 * * *$

\begin{tabular}{|c|c|c|}
\hline $\begin{array}{c}\text { Disability } \\
\text { status }\end{array}$ & Males: $-6.304^{* * *}$ & $\begin{array}{c}\text { Psychosocial distress } \\
\text { (MHI5 total score) }\end{array}$ \\
\hline
\end{tabular}

$3 \mathbf{b}$

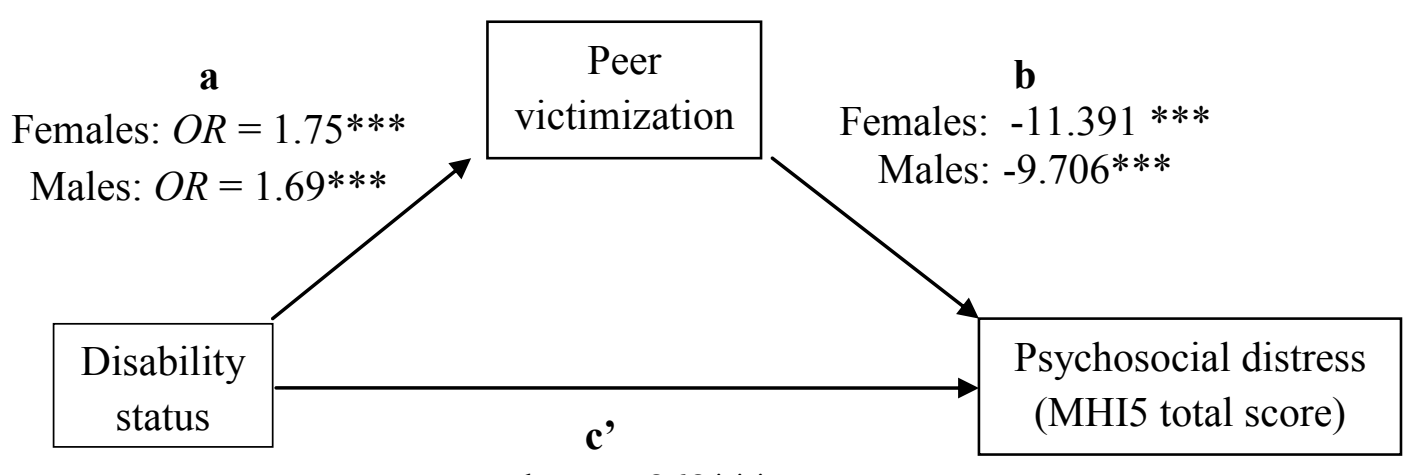

Females: $-5.868 * * *$

Males: $-5.221 * * *$

\section{Figure 3.}

Mediation results. Unstandardized regression coefficients for the relationship between disability status and psychosocial distress as mediated by exposure to peer victimization in the past 30 days for females and males. Odds ratios are reported for path a instead of unstandardized coefficients. $* * * p<.001$. 
Table 22

Regression Results from Mediation Analyses ${ }^{\text {ab }}$

\begin{tabular}{|c|c|c|c|c|c|}
\hline \multirow{2}{*}{ Path/effect by Groups } & \multirow{2}{*}{$\beta$} & \multirow{2}{*}{$S E$} & \multirow{2}{*}{$t$} & \multicolumn{2}{|c|}{$95 \% \mathrm{CI}$} \\
\hline & & & & LL & UL \\
\hline \multicolumn{6}{|l|}{ Females $(n=3492)$} \\
\hline $\mathrm{c}(\mathrm{DA} \rightarrow \mathrm{PD}) * * *$ & -7.333 & 0.753 & -9.74 & -8.83 & -5.83 \\
\hline $\mathrm{a}(\mathrm{DA} \rightarrow \mathrm{PV}) * * *$ & 0.562 & 0.059 & 9.47 & 0.44 & 0.68 \\
\hline $\begin{array}{l}\mathrm{a}(\mathrm{DA} \rightarrow \mathrm{PV}) \\
(\text { standardized beta })\end{array}$ & 0.134 & 0.014 & & & \\
\hline $\mathrm{b}(\mathrm{PV} \rightarrow \mathrm{PD}) * * *$ & -11.391 & 0.776 & -14.68 & -12.94 & -9.84 \\
\hline $\begin{array}{l}\mathrm{c}^{\prime}(\mathrm{DA} \rightarrow \mathrm{PD}, \text { controlling for } \\
\mathrm{PV})^{* * *}\end{array}$ & -5.868 & 0.746 & -7.87 & -7.36 & -4.38 \\
\hline Mediated effect estimate $(a b)^{c}$ & -1.523 & 0.191 & & -1.9 & -1.15 \\
\hline \multicolumn{6}{|l|}{ Males $(n=3172)$} \\
\hline $\mathrm{c}(\mathrm{DA} \rightarrow \mathrm{PD}) * * *$ & -6.304 & 0.959 & -6.57 & -8.22 & -4.39 \\
\hline $\mathrm{a}(\mathrm{DA} \rightarrow \mathrm{PV}) * * *$ & 0.526 & 0.082 & 6.4 & 0.36 & 0.69 \\
\hline $\begin{array}{l}\text { a (DA } \rightarrow \text { PV) (standardized } \\
\text { beta) }\end{array}$ & 0.119 & 0.019 & & & \\
\hline $\mathrm{b}(\mathrm{PV} \rightarrow \mathrm{PD}) * * *$ & -9.706 & 0.793 & -12.24 & -11.29 & -8.12 \\
\hline $\begin{array}{l}\mathrm{c}^{\prime}(\mathrm{DA} \rightarrow \mathrm{PD}, \text { controlling for } \\
\mathrm{PV}) * * *\end{array}$ & -5.221 & 0.916 & -5.7 & -7.05 & -3.39 \\
\hline Mediated effect estimate $(a b)^{c}$ & -1.154 & 0.203 & & -1.55 & -0.76 \\
\hline
\end{tabular}

Note. $\mathrm{CI}=$ confidence interval; $\mathrm{LL}=$ lower limit; $\mathrm{UL}=$ upper limit. Significance results reported are based upon design-based tests using the Wald method. ${ }^{\mathrm{a}}$ Model and regression estimates significant at $\mathrm{p}<0.000$; females: $F(2,67)=$ 127.81; males: $F(2,67)=114.25$. ${ }^{\mathrm{b}} \mathrm{DA}=$ disability $(0=$ non-disabled, $1=$ disabled $)$, $\mathrm{PD}=$ psychosocial distress (a higher score indicated better mental health), $\mathrm{PV}=$ peer victimization $(0=$ not exposed, $1=$ exposed $)$. ${ }^{c}$ ab calculated with standardized beta for path a and unstandardized beta for path $b$.

$* * * \mathrm{p}<.001$ 
Table 23

Estimated Proportion Mediated and Total Effects

\begin{tabular}{cc}
\hline Equation & Estimate \\
\hline Females $(n=3492)$ & \\
Proportion mediated: & \\
$1-\left(c^{\prime} / \mathrm{c}\right)$ & 0.191 \\
$\mathrm{ab} / \mathrm{c}$ & 0.210 \\
$\mathrm{ab} /(\mathrm{c}+\mathrm{ab})$ & 0.174 \\
Total effect & \\
$\mathrm{c}^{\prime}+\mathrm{ab}$ & -7.391 \\
$\mathrm{c}$ & -7.333 \\
Males $(n=3492)$ & \\
Proportion mediated: & \\
$1-\left(c^{\prime} / \mathrm{c}\right)$ & 0.172 \\
ab / c & 0.183 \\
ab / (c + ab) & 0.155 \\
Total effect & \\
$\mathrm{c}^{\prime}+\mathrm{ab}$ & -6.375 \\
$\mathrm{c}$ & -6.304 \\
\hline
\end{tabular}

Note: Due to the mix of logistic and linear regression, the proportion mediated and total effects will not be identical, but similar, after standardizing the logistic estimate (path a). 


\section{CHAPTER VI: DISCUSSION}

In this chapter, I summarize the findings for the two distinct studies contained within this dissertation, and discuss the degree to which these findings are in concordance with the empirical and theoretical literature review. In doing so, I will make the case that the results from both studies suggests the need to consider the role of "disability" status as a marker of "difference." I then discuss the limitations of this research. The theoretical implications from this dissertation research will next be discussed, followed by implications for practice, policy and research. Suggestions for future research and a brief conclusion complete this chapter.

\section{Summary of Findings}

Most of the study hypotheses were supported related to the intersections of disability, sex, LGB, and race/ethnicity statuses in predicting exposure to peer victimization. Not only were youth with disabilities more likely to report exposure to peer victimization, compared to youth without disabilities (Table 18, Model 1), the relative magnitude of exposure to peer victimization increased when disability status was combined with sex, race/ethnicity or sexual orientation (Table 18, Models 2-4). The degree of the magnitude of odds of exposure increased markedly when disability status was combined with race/ethnicity and sex, or sexual orientation and sex (Table 18, Models 5-6). In general, significant differences were found between the subgroups at both ends of spectrum (e.g., differences between non-disabled white females compared to disabled males of color), but this was not always the case. For example, the odds of exposure to peer victimization in subgroups which differed only by sex were not found to 
significantly differ between non-disabled males of color and non-disabled females of color.

Peer victimization was shown to be a partial mediator of the relationship between disability status and psychosocial distress. However, while sex was a significant predictor of both peer victimization and psychosocial distress, sex did not significantly moderate the mediating effects of peer victimization on the relationship between disability status and psychosocial distress. In other words, psychosocial distress reported by males and females with disabilities exposed to peer victimization may not be all that different.

\section{Concordance}

Most of the findings from this dissertation research are in agreement with the empirical and theoretical review. First, the results from this study are in alignment with the various "gradients" detected in the empirical literature review, particularly for females, females with disabilities and GBQ males. Specifically, the higher rate of exposure to peer victimization among females compared to males was consistent with previous findings (Eaton et al., 2005; Nabuzoka et al., 2009; Sawyer et al., 2008). Differences by sex and sexual orientation statuses also were consistent with the empirical literature reviewed (Berlan et al., 2010; Friedman et al., 2011; Kosciw et al., 2010).

Many of the studies reviewed suggest that the percentage of students reporting peer victimization may be roughly the same for both males and females (although the type of peer victimization is often different); results from this study indicated significant, but relatively small differences by sex alone. Yet, the results of this study add another dimension to the knowledge base; sex differences in prevalence rates do exist, but they 
stand out more when considered in conjunction with another axis of diversity such as disability or sexual orientation. For example, the odds of exposure to peer victimization among females with disabilities were twice that of the odds of disabled males reporting peer victimization. However, this is not always the case. In this study, the odds of exposure to peer victimization for disabled males of color and disabled females of color were nearly identical. In the context of this study, sex matters, especially in combination with disability status, except among students of color.

Results from this research study add to the mixed findings in the literature when it comes to examining the prevalence of peer victimization by race/ethnicity. White males and females were less likely to report peer victimization compared to students of color, which is not consistent with other findings; this is especially true with respect to black students who are generally reported in the literature as less likely to report peer victimization compared to their white peers (e.g., Hanish \& Guerra, 2000; Juvonen, Graham, \& Schuster, 2003; Sawyer et al., 2008; Spriggs, Iannotti, Nansel, \& Haynie, 2007; Wang et al., 2010). The methodology used in this study may help explain the nature of inconsistent finding in the literature; dichotomizing the racial/ethnic categories prior to analysis may have masked within subgroup differences among students of color. Yet with an intersectional lens, the findings from this study revealed sex differences when combined with race/ethnicity and disability, particularly when comparing exposure to peer victimization by disabled students of color to that of non-disabled students of color, and to non-disabled white students. 
The findings in this study were also consistent with another type of gradient observed in the literature, in which students with disabilities reported greater levels of psychosocial distress, with psychosocial distress greatest for females with disabilities (Miauton et al., 2003, Wolman et al., 1994). However, as mentioned above, while sex was found to be significantly associated with increased psychosocial distress and peer victimization, sex was not shown to moderate the effects of peer victimization on psychosocial distress among students with disabilities.

\section{Limitations}

Several limitations of this dissertation research must be considered when interpreting and generalizing the findings, particularly with regard to survey design, methodology, and analyses.

Survey design. An obvious limitation is the problem of self-report on surveys, particularly on sensitive items, such as querying youth about sexual identity and sexual behavior, or items reflecting issues of social desirability (such as exposure to peer victimization). This problem can produce a non-response bias, as was the case in this study, particularly in regards to youth from non-dominant groups not answering the question about harassment, which made it difficult to impute missing responses (methodologically there are ways to impute missing response found among the independent variables, the same is not true for dependent variables). While a limitation, the problem of non-response bias is intriguing and may well be quite relevant to this study, as students with other non-dominant identities were also more likely not to answer the questions about sexual identity and sexual behavior. 
The non-response bias may be influenced also by the design of the questions used to query students' disability status, and sexual orientation. These questions often inevitably reflect dominant culture bias and may not adequately reflect the identities or social labels preferred by students. The structure of these questions often assume homogeneity in identity labels (e.g., gay/lesbian/ bisexual/ heterosexual; male/female). Such assumed homogeneity and lack specificity in questions makes it more difficult for researchers to study the association between a social identity or characteristic, and exposure to peer victimization or other detrimental health risks. For example, combining the question about problems hearing with problems seeing ignores the differences in the social experiences by deaf/hard of hearing students and those with low vision or who are legally blind. Another example of reduced specificity is in the collapsing of learning disabilities with ADHD and other cognitive disabilities.

More concerning, however, is how these questions may perpetuate stigmatization and introduce additional social desirability bias. For example, in the question asking about learning disabilities, the question included the pejorative term of "mental retardation" in querying students about learning disabilities. Even the placement of the questions on the survey is problematic. For example, the two questions related to sexual orientation status were placed under the category of sexual behavior, rather than presented as a demographic question. Likewise, questions about disability status were not presented as demographic questions, but instead, as questions about "other health conditions." Treating these questions as normative demographic questions may reduce social desirability bias associated with disability. 
A common limitation in conducting secondary data analysis is using an existing dataset not designed for the particular research project. While the survey was intended to be multi-purpose in regards to assessing health risks (Brener et al., 2004), it was not designed to examine the relationship of psychosocial health in regards to known risk factors of health, such as peer victimization. Nor did it focus on examining prevalence of peer victimization by types of victimization experienced. Finally, another limitation specific to the survey design concerns youth who are not represented in the survey. Specifically, youth not in school and youth with disabilities who were not invited to complete, or may not have completed, the survey because they needed accommodations may not have been fully included.

Methodology and analyses. Some of the limitations mentioned above related to the type of measures available to me, in turn limited the analyses I could do. A key limitation was the peer victimization variable, which as a dichotomized mediator was very difficult to test in combination with estimate restrictions due to the nature of the complex survey data. Also, due to lack of access to school level variables, I was unable to examine the extent to which school-level variables were associated with prevalence of peer victimization.

The research findings are constrained with the collapsing of students of color, as well as students with disabilities, into single categories. Such collapsing can masks within group differences; caution is needed in generalizing these results. The mediation findings are also limited due to the exclusion of many other factors, which are known to be 
associated with psychosocial distress and were not included in the model (e.g., low selfesteem, poor grades, child abuse).

A number of students elected not to answer questions relevant to this study, which produced sizable missing responses, particularly in regards to sexual orientation and exposure to peer victimization; as a consequence, logistic analyses were conducted using list-wise deletion which reduced the sample size considerably, from 7091 in the initial sample to 6045. That alone, however, would not have been a concern except for the intersectional approach taken in this survey. The intersectional approach relied upon small cell sizes among students with multiple non-dominant social statuses; in the case of this study, small cell sizes produced a relatively high standard error in regards to disabled GBQ males $(S E=3.31)$.

Despite these limitations, analyses of this population-based survey were informative. Results from this study demonstrates the value of quantitative intersectional analyses, particularly in the context of examining who are most likely to report peer victimization. Also we have a better understanding the degree of psychosocial distress reported by students with disabilities in mainstream classrooms. Below I discussed in detail the implications for theory, practice, policy, and future research.

\section{Implications}

In this section I will discuss the findings from this study in terms of the theoretical, practice, policy, and research implications; many of these implications will be couched in terms of strategies to reduce exposure to peer victimization, and to improve the psychosocial health of students with disabilities. However, before proceeding to the 
specific implications (and strategies), it may be helpful to consider in depth several issues that, if not critically examined, could inadvertently render actions resulting from these implications ineffective.

The first danger is that of essentializing social categories and binaries, and in effect "writing out the social" (Brown, 2012). While this is an intersectional study, it was done in the post-positivist tradition, guided in large part by how we socially construct and make sense of differences, whether it be by social identities (disability, sex, race/ethnicity, sexual orientation), and who commits - and who experiences - the violence. Yet, as aptly stated by Moy (2008) in her analysis of the "bully discourse," "these fixed categories within a simplistic framework that focuses on binaries (weak/strong, passive/aggressive) whitewashes (sic) any reference to complex social identities" (Moy, 2008, p. 70). This leads to the second danger: the inadvertent shift from characterizing the problem as a symptom of larger structural oppressions that maintain an oppressive hierarchy, to the individual level in which the "bully" gets the spotlight (Moy, 2008). Indeed, it is easier to suggest and articulate individual level strategies, rather than focusing on macro level structures that maintain hierarchical oppression. An insidious consequence of the focus on the individual with easily understood categories, via the "bully/victim" discourse, is victim blaming (passive, aggressive, socially inappropriate), and characterizing the "bully" as someone who is different from the rest of us. In actuality, I suspect, we have all harmed another peer, and we have all experienced peer victimization. 
Therefore, in writing this section on implications, I tried to be mindful of these dangers. And, as someone with a fondness for organizing frameworks and categories, I am reminded and reassured that "the act of categorization is not in itself so troubling. Rather, social categories are problematic in the extent to which they become preordained, imposed, and hegemonic" (Anderson-Nathe, Gringeri, \& Wahab, 2013, p. 288). This is context in which I present these implications.

Theoretical implications. The findings of this dissertation have important implications related to the theoretical model outlined in Chapter Two. Before I proceed to discuss these implications, however, I need to explain an additional limitation. This study did not explore access to and use of coping resources and support systems, nor the degree to which identity was related to internal stress processes. Therefore, implications for Meyer's minority stress model (2003) are limited to the extent to which non-conformity produces greater vulnerability to peer victimization. Further, based upon the mediation results from this research, exposure to such stressors is associated with psychosocial distress. With this limitation acknowledged, I now proceed to discuss implications for the theoretical framework.

As mentioned in Chapter Three, a lack of fit of the individual with the social environment and perceived or real social vulnerability, combined with a power differential between the parties involved, is thought to predict greater exposure to peer victimization (Juvonen \& Galván, 2009; Olweus, 1993; Orpinas \& Horne, 2005). This can be seen in the case of youth with disabilities, for example, who were consistently 
more likely to report peer victimization compared to non-disabled students, either alone or in combination with one other status.

The poor fit with the social environment for youth with disabilities may be reflected in the significantly greater rates of peer victimization reported by students with disabilities, compared to non-disabled students, resulting in greater psychosocial distress. It is noteworthy that in other studies, sex is seen to moderate the mediating effects of peer victimization on psychosocial distress; this was not the case for students with disabilities. This finding raises an important theoretical question: is disability gendered to the extent that sex differences in the prevalence of psychosocial distress are minimized? This is an area where disability studies can lend further understanding to gender studies.

In this research, the complexity in regards to the lack of fit among students with multiple non-dominant statuses is reflected in the patterning of differential exposure to peer victimization. The magnitude of differences in exposure to peer victimization increased considerably for disabled student of color; $49 \%$ of male and female disabled students of color (male and female) reported peer victimization, compared to $23.9 \%$ of white disabled males, and $40.6 \%$ of white disabled females. The magnitude of differences was especially marked for LGBQ youth with disabilities; $75 \%$ of GBQ males with disabilities reported exposure to peer victimization in the past 30 days, compared to $53 \%$ of non-disabled GBQ males. Clearly, the relative magnitude of exposure to peer victimization cannot be characterized as additive or multiplicative ("double" or "triple jeopardy"), as the type of non-dominant and dominant culture status is more informative than the number of non-dominant statuses held by the student. 
Power, real or perceived, may help explain the magnitude of oppression which varied by the combination of social identities. I am speaking specifically about the type of power that either protects or makes one more vulnerable to the experience of victimization. This real or perceived power could embodied in the individual (e.g., physical strength), or ascribed to an individual due to one's social status. In this study, with the focus on systematic exposures to peer victimization, the lack of power one experiences as a member of a non-dominant group in combination with one's physical power or ability, could make it easier for members of dominant groups to keep members of certain groups “in," "out” or "down." This power differential may be particularly salient for people with physical disabilities (compared to non-disabled people), and for females (compared to males). This real or perceived weakness may make it easier for those who are stronger, and who have the support from dominant culture norms, to victimize. Indeed, findings from this research revealed that disability status was a stronger predictor of exposure to peer victimization than race or sex. Is this because students with disabilities are generally perceived as weaker and less powerful, and in combination with dominant culture norms which values ability (and appearances), makes it easier for people with disabilities as a group to be victimized? However, for students with invisible disabilities, students of color, and GBQ males, this perceived or real weakness cannot be assumed.

Here, however, is where I am compelled to remind the reader that theorizing of power exclusively at an individual level or group level, runs the risk of shifting the gaze away from the larger systems that make it easier for individuals to engage in such 
behaviors. Rather, it may be more productive to look at individuals within a group supported by dominant culture norms, which maintains an oppressive hierarchy that goes well beyond the individual level. In this respect, Phelan, Link and Dovidio's (2008) typology might serve as a theoretical framework to explain differential exposures to peer victimization. In other words, how is it that the person doing the victimizing is supported by dominant culture norms? Are certain dominant culture norms more rigid than others, which would, for example, explain increased exposure to violence among disabled male GBQ students versus disabled males who identify as straight?

Practice implications. Findings from this study can inform future efforts to address the problem of peer victimization among all youth, including youth with disabilities. Practitioners, advocates, and others can utilize an intersectional lens to broaden efforts to address the problem of violence in schools and communities. Specifically, understanding the idea of a "gradient of risks" which varies by an individual's social location at school, in regards to disability, race/ethnicity, sexual orientation, and sex, can help to recognize and highlight those who are "in the margins" and are most likely to experience peer victimization. Such interventions will be more effective if they stretch to focus on the conditions at the macro-mezzo level that maintain systems of oppression. For example, instead of focusing solely on individual level strategies, we could focus on the problem of systematic oppression, and how this problem is supported by the socio-cultural context in which we live. Such an approach will make it more likely that attention is given to the problem of peer victimization experienced by all youth, including youth with disabilities. It is important that social workers, school 
counselors, teachers and others view youth with disabilities as belonging to a nondominant (cultural) group experiencing higher rates of peer victimization.

Social workers, parents, advocates and others concerned with the mental health of students may want to consider Meyer's minority stress model as (2003) applicable to disabled students, as well as to other students holding one or more non-dominant culture identity/statuses. The Meyer's minority stress model helps to frame perceived studentlevel characteristics, such as disability status, as influencing the "fit" between the individual and the social environment. Not only does this model explicitly situate how one's non-dominant culture status might result in increased exposure to violence, this model also highlights the importance of identity development, access to and usage of coping and support resources, and how one understands the experience of peer victimization.

The Meyer's minority stress model could be helpful in addressing both individual level strategies, and school and community level strategies that go beyond the individual level to the group/school/community level. Specifically, at an individual level with students with disabilities, instead of assuming that disability status causes psychosocial distress, thus contributing to the internalization of disablism among students, or blaming the disabled student for being too passive or provocative, the social worker could help the student to understand how one's social location makes it more likely for one to be a target of systematic oppression.

Findings from this research suggest that social workers and others concerned with the social health of students will be more effective in their efforts if they work across 
groups to include youth "in the margins." Doing so requires recognizing the diversity within groups, and looking for opportunities that go beyond the binaries. This includes diversity by sex, as findings from this research suggest one should not disregard the disabled males who experience peer victimization. For example, strategies that might reach non-disabled LGBQ youth, such as a Gay Straight Alliance (GSA), are not necessarily inclusive of LGBQ youth with disabilities; a hierarchy based upon ability (even within non-dominant sexual orientation) exists. Advocates and student leaders are encouraged to examine the extent to which LGBQ youth with disabilities are actively welcomed and included in GSA activities. Indeed, organizations, whether at the school or in the community, similar to a GSA, can do much to counteract the notion that disabled LGBTQ students do not exist, or somehow do not want/need the supports from such an organization. At the same time, these types of organizations, particularly those lead by students, provide the needed individual level supports to youth who are living in an oppressive society. Such actions could benefit all students, not just those holding one non-dominant culture identity, but also those holding multiple non-dominant culture identities.

It is time to bring disability, as a non-dominant social identity, into focus; it will be most effective, however, to do this using an intersectional approach. All too often, as noted by Moy (2008), "the relative exclusion of ableism from central discussion of identity and violence was common in numerous interviews which positioned race, gender, homophobia (and to a lesser extent, class) as the major 'bastions' of prejudice and violence" (p. 93). Taking an intersectional lens, social workers, advocates, teachers, 
and others could facilitate critical dialogue that explicitly calls out the problem of peer victimization by those with non-dominant culture identities, including students with disabilities.

Policy implications. The patterning of exposure to peer victimization suggests strategies going beyond a focus on individual students in interventions. If, as suggested by this research, exposure to peer victimization is highly associated with a group of students with one or more non-dominant culture statuses, then strategies going beyond the individual will be more effective in preventing and reducing exposure to peer victimization (Hatzenbuehler, Phelan, \& Link, 2013). Policies addressing violence experienced by members of non-dominant cultures, but not those with disabilities, are denying the existence of an oppressive hierarchy (Burke \& Eichler, 2006). Acknowledging such hierarchy is an essential first step towards eliminating an oppressive bias.

The patterning of exposure to peer victimization suggests a need for advocates and policy makers to work across groups to craft policies with attention to youth "in the margins" who are most affected by peer victimization. It is crucial that advocates and policy makers incorporate an intersectional perspective, making visible not only those with disabilities, but others who hold non-dominant statuses within their communities. Policies that do not explicitly acknowledge both the disproportionate exposure to peer victimization by social statuses, and the hierarchy of exposure by social statuses, maintains an oppressive hierarchy. 
Research implications. Taking an intersectional approach in research can help avoid bias that can result from a focus on one social category or another, without regard to differences between or within groups. Examining prevalence of peer victimization by a single axis of social status fails to explicate the variance within and between groups. For instance, had I only examined the prevalence of peer victimization by disability status, I would have missed the patterning of exposures to peer victimization by social statuses, and the fact that the relative magnitude of exposure to peer victimization varied considerably with the addition of sex and sexual orientation together. Likewise, if I had only examined the prevalence of peer victimization among LGBQ students, the high rate of peer victimization among GBQ males with disabilities would have been missed. In both cases, failing to examine differences between or within subpopulations delineated by demographic characteristics constitutes bias in research. Such bias can perpetuate an oppressive hierarchy (Burke \& Eichler, 2006) where those who are most vulnerable to violence are kept invisible. One's social location in high school is an important predictor of exposure to peer victimization, and cannot be adequately described in terms of one social characteristic or identity at a time.

The analyses that examined peer victimization as a mediator of the relationship between disability status and psychosocial distress suggest a need for researchers to consider "disability" status as a marker of "difference." In this case, the embodied impairment in the individual heightens the risk of exposure to disablism, the discrimination based on disability status or functional impairment. Situating disability 
status in this manner opens up lines of inquiry that create ways to deepen understanding of issues related to social justice and people with disabilities.

\section{Suggestions for Future Research}

This chapter includes a call for research to help identify and understand, to the extent that it is possible, how and why some youth, particularly those with non-dominant statuses/identities, are more likely to be exposed to peer victimization in high school. Below I describe a number of inquiries that could be undertaken by researchers, policy makers, social workers, and others that could reduce the incidence of peer victimization in high school.

Stigma, peer victimization, and health inequities. The results support a focus on stigma as a social determinant of population health (Hatzenbuehler et al., 2013). Specifically, disablism resulting from stigma and prejudice, theorized by Phelan, Link and Dovidio (2008) to be rooted in "disease avoidance," keeps people with disabilities out; this is done both structurally at a macro level, and by interpersonal interactions at a micro level. The question to consider is why the effects of disablism are more severe, resulting in a higher rate of peer victimization, than the effects of sexism or racism. And, should the application of the Meyer's (2003) minority stress model be considered by the particular function(s) of oppression experienced by the group of interest?

There is a need to explore further how power, or lack of power due to stigmatization, connects to the gradient in exposure to peer victimization found in this research. Is the behavior by those victimizing others purposive, and on the basis of a non-dominant culture status? To what extent is the individual, who victimizes others, intent upon 
maintaining hierarchy? Is the relative high magnitude of exposure among LGBQ students a reflection of how non-compliance with social norms is punished more severely than simply being "different"? To what extent does the power and privilege of the person doing the victimizing, relative to the person being victimized, enable the manifestation of oppression? And at a macro level, to what extent does denial of hierarchy, and passive acceptance of peer victimization, differ based upon certain non-dominant statuses, and the combination of such? It would also be helpful to explore further if differential power within and between groups, in the context of peer victimization in high school, is understood more easily if conceptualized in terms of sexuality and gender roles. I suggest this due to the different patterning of exposure to peer victimization among LGBQ students. However, in order to get to this, we must consider the diversity within and between groups; taking an intersectional approach can help do this.

Utilizing an intersectional lens in research. Research is needed to examine the prevalence of peer victimization among youth "in the margins," going beyond the binaries. In this research, I did not employ an intersectional lens in the testing of mediation and moderated mediation; future research could do exactly that and perhaps take it further by testing Meyer's (2003) minority stress model. In other words, research could examine how the fit between the individual and the environment can be enhanced (e.g., with social supports centered on multiple status groupings or working across groups). Research employing an intersectional lens, regardless of the methodology, will provide a keener understanding of the problem of peer victimization and guide practice and policy interventions. However, it is not sufficient to simply "do intersectional" 
research; the researcher must determine what makes sense in thinking of social categories. Below I suggest future research based upon my own thinking of these social categories in the context of peer victimization and the results of this dissertation research, and conclude this chapter with suggestions for future survey research.

The intersectional approach could be helpful in exploring further the role of sex and gender roles as disability is thought to be "gendered," meaning that societal expectations regarding masculinity and femininity are attenuated with regard to people with disabilities (Garland-Thompson, 2004; Gerschick, 2000; Shuttleworth, 2004). The gendering of disability may partially explain why study findings did not support significant sex differences in psychosocial distress among disabled students who experienced peer victimization. This may be because sex in combination with disability status diminishes differences by sex, compared to sex differences among people without disabilities. It could be illuminating to understand better how the influence of nondominant statuses due to sex and disability differs from the influence of sex and other non-dominant groups.

Survey research. More survey research is needed with disability status as a demographic variable. In addition to disability status, there is a need for population-based surveys to situate non-dominant statuses as demographic variables and not, for example, as outcomes in the case of disability status, or as a behavior, in the case of sexual orientation. These steps would make it easier to utilize an intersectional approach in research. Surveys that allow specificity by disability type and that use identity language 
favored by youth with disabilities and other marginalized statuses will contribute to our understanding of the experienced of these youth.

A possible non-response bias emerged in this dissertation research related to students answering questions about sexual orientation and peer victimization, particularly among those with more than one non-dominant status. Research could be helpful to understand the relationship of non-response on sensitive survey items and multiple stigmatized identities; as well as how such bias can be minimized on future surveys.

\section{Conclusion}

As I bring this dissertation study to a close, I am compelled to describe five ways in which this research is innovative. First, this is the first known study testing the role of peer victimization as a mediator on the relationship between disability status and psychosocial distress, using a representative sample which included a relatively large percent of disabled students. Remarkably, studies such as this are absent when it comes to students with disabilities, perhaps due to the neglect or tolerance of peer victimization among students with disability in the peer victimization discourse (Moy, 2008). Second, this is the first known study to systematically examine exposure to peer victimization when disability is included with other statues. Third, I was able to show the value of taking an intersectional approach as the findings revealed the degree to which exposure to peer victimization varied by the student's social status; this approach provided a better understanding of who, among 11th grade students in Oregon, are more at risk for exposure to peer victimization. Fourth, as demonstrated earlier in this chapter, I showed how the results from this study can be used to understand any discordance or patterns in 
the empirical literature, and how interpreting the results in the context of the intersections of multiple non-dominant statuses, power and non-conformity, can potentially lead to a deeper understanding of the variance in the prevalence of peer victimization by social statuses. Finally, I demonstrated how to take an intersectional approach to research involving population-based surveys.

The primary motivation for conducting this study stemmed from wanting to explore the benefits in using an intersectional approach to bringing attention to the problem of disablism experienced by high school students with disabilities, which in this case is exposure to peer victimization. I hope I was able to accomplish this task without diminishing the effects of racism, heterosexism, and sexism. I also hope that future research will employ similar methodological approaches that might help bring diverse communities together to address the social problem of peer victimization and other forms of oppression. 


\section{REFERENCES}

Allport, G. W. (1954). The nature of prejudice. Garden City, NY: Doubleday.

Alriksson-Schmidt, A. I., Armour, B. S., \& Thibadeau, J. K. (2010). Are adolescent girls with a physical disability at increased risk for sexual violence? Journal of School Health, 80(7), 361-367.

Altman, B. M. (2001). Disability definitions, models, classification schemes, and applications. In G. L. Albrecht, K. D. Seelman \& M. Bury (Eds.), Handbook of disability studies (pp. 97-122). Thousand Oaks, CA.: Sage Publications.

Anderson-Nathe, B., Gringeri, C., \& Wahab, S. (2013). Nurturing "Critical Hope” in teaching Feminist social work research. Journal of Social Work Education, 49(2), 277-291. doi: 10.1080/10437797.2013.768477

Archer, K. J., Lemeshow, S., \& Hosmer, D. W. (2007). Goodness-of-fit tests for logistic regression models when data are collected using a complex sampling design. Computational Statistics \& Data Analysis, 51(9), 4450-4464. doi: http://dx.doi.org/10.1016/j.csda.2006.07.006

Babbie, E. (1990). Survey research methods (2nd ed.). Belmont, CA: Wadsworth Publishing Company.

Baker, K., \& Donelly, M. (2001). The social experiences of children with disability and the influence of environment: A framework for intervention. Disability \& Society, 16(1), 71-85. doi: 10.1080/09687590020020877

Baron, R. M., \& Kenny, D. A. (1986). The moderator-mediator variable distinction in social psychological research: Conceptual, strategic, and statistical considerations. Journal of Personality and Social Psychology, 51(6), 1173-1182.

Baumeister, A., Storch, E., \& Geffken, G. (2008). Peer victimization in children with learning disabilities. Child \& Adolescent Social Work Journal, 25(1), 11-23. doi: 10.1007/s10560-007-0109-6 
Berlan, E. D., Corliss, H. L., Field, A. E., Goodman, E., \& Austin, S. B. (2010). Sexual orientation and bullying among adolescents in the Growing Up Today study. The Journal of Adolescent Health, 46(4), 366-371.

Birkett, M., Espelage, D., \& Koenig, B. (2009). LGB and questioning students in schools: The moderating effects of homophobic bullying and school climate on negative outcomes. Journal of Youth and Adolescence, 38(7), 989-1000.

Blum, R. W., Kelly, A., \& Ireland, M. (2001). Health-risk behaviors and protective factors among adolescents with mobility impairments and learning and emotional disabilities. The Journal of Adolescent Health, 28(6), 481-490.

Blum, R. W., Resnick, M. D., Nelson, R., \& St Germaine, A. (1991). Family and peer issues among adolescents with spina bifida and cerebral palsy. Pediatrics, $88(2)$, 280-285.

Bontempo, D., E., \& D'Augelli, A., R. (2002). Effects of at-school victimization and sexual orientation on lesbian, gay, or bisexual youths' health risk behavior. The Journal of Adolescent Health, 30(5), 364-374.

Bowleg, L. (2008). When black + lesbian + woman $\neq$ black lesbian woman: The methodological challenges of qualitative and quantitative intersectionality research. Sex Roles, 59(5/6), 312-325. doi: 10.1007/s11199-008-9400-z

Brener, N. D., Kann, L., Kinchen, S. A., Grunbaum, J. A., Whalen, L., Eaton, D. (2004). Methodology of the youth risk behavior surveillance system. MMWR. Recommendations And Reports: Morbidity And Mortality Weekly Report, 53(RR12), 1-13.

Brown, C. G. (2012). Anti-oppression through a postmodern lens: Dismantling the master's conceptual tools in discursive social work practice. Critical Social Work, 13(1), 34-65.

Brunner, E., \& Marmot, M. (2006). Social organization, stress and health. In M. Marmot \& R. Wilkinson (Eds.), Social determinants of health (2nd ed., pp. 6-30). NY: Oxford University Press. 
Burke, M. A., \& Eichler, M. (2006). The BIAS FREE Framework: A practical tool for identifying and eliminating social biases in health research. http://www.biasfree.org/full_BF.pdf

Campbell, C., \& Deacon, H. (2006). Unravelling the contexts of stigma: From internalisation to resistance to change. Journal of Community \& Applied Social Psychology, 16(6), 411-417.

Carroll, P., \& Shute, R. (2005). School peer victimization of young people with craniofacial conditions: A comparative study. Psychology, Health \& Medicine, 10(3), 291-304. doi: 10.1080/13548500500093753

Centers for Disease Control and Prevention. (2005). 2005 National YRBS data users manual.

Chandra, A., Mosher, W. D., Copen, C., \& Sionean, C. (2011). Sexual behavior, sexual attraction, and sexual identity in the United States: Data from the 2006-2008 National Survey of Family Growth. Hyattsville, MD: Centers for Disease Control and Prevention Retrieved from http://www.cdc.gov/nchs/data/nhsr/nhsr036.pdf.

Cochran, S. D. (2001). Emerging issues in research on lesbians' and gay men's mental health: Does sexual orientation really matter? American Psychologist, 56(11), 931-947.

Cole, E. R. (2009). Intersectionality and research in psychology. American Psychologist, 64(3), 170-180. doi: 10.1037/a0014564

Commission on Social Determinants of Health. (2008). Closing the gap in a generation: Health equity through action on the social determinants of health. Final report of the Commission on Social Determinants of Health. http://whqlibdoc.who.int/publications/2008/9789241563703_eng.pdf

Crenshaw, K. (1991). Mapping the margins: Intersectionality, identity politics, and violence against women of color. Stanford Law Review, 43(6), 1241-1299.

Cuijpers, P., Smits, N., Donker, T., ten Have, M., \& de Graaf, R. (2009). Screening for mood and anxiety disorders with the five-item, the three-item, and the two-item 
Mental Health Inventory. Psychiatry Research, 168(3), 250-255. doi: 10.1016/j.psychres.2008.05.012

D'Augelli, A. R., \& Hershberger, S. L. (1993). Lesbian, gay, and bisexual youth in community settings: Personal challenges and mental health problems. American Journal of Community Psychology, 21(4), 421-448. doi: 10.1007/bf00942151

Daley, A., Solomon, S., Newman, P. A., \& Mishna, F. (2007). Traversing the margins: Intersectionalities in the bullying of lesbian, gay, bisexual and transgender youth. Journal of Gay \& Lesbian Social Services, 19(3/4), 9-29.

Dao, T. K., Kerbs, J. J., Rollin, S. A., Potts, I., Gutierrez, R., Choi, K. (2006). The association between bullying dynamics and psychological distress. The Journal of Adolescent Health, 39(2), 277-282.

Davis, K. (2008). Intersectionality as buzzword: A sociology of science perspective on what makes a feminist theory successful. Feminist Theory, 9(1), 67-85.

Dawkins, J. L. (1996). Bullying, physical disability and the paediatric patient. Developmental Medicine \& Child Neurology, 38(7), 603-612.

Demidenko, E. (2007a). Power/Sample Size Calculation for Logistic Regression with Binary Covariate(s) (Online power calculator). Retrieved 4/16/11, from Dartmouth Medical School http://www.dartmouth.edu/ eugened/powersamplesize.php

Demidenko, E. (2007b). Sample size determination for logistic regression revisited. Statistics in Medicine, 26(18), 3385-3397.

Doubt, L., \& McColl, M. A. (2003). A secondary guy: Physically disabled teenagers in secondary schools. Canadian Journal of Occupational Therapy, 70(3), 139-151.

Dovidio, J. F. (2001). On the nature of contemporary prejudice: The third wave. Journal of Social Issues, 57(4), 829-849. doi: 10.1111/0022-4537.00244

Eaton, D., K., Kann, L., Kinchen, S., Shanklin, S., Ross, J., Hawkins, J. (2010). Youth Risk Behavior Surveillance: United States, 2009. Morbidity and Mortality Weekly Report, 59(SS-5), 1-142. http://www.cdc.gov/mmwr/pdf/ss/ss5905.pdf 
Eaton, D., K., Kann, L., Kinchen, S. A., Ross, J. G., Hawker, D., Harris, W. A. (2005). Youth Risk Behaviour Surveillance: United States, 2005. Atlanta: Centers for Diesase Control and Prevention Retrieved from http://www.cdc.gov/mmwr/preview/mmwrhtml/ss5505a1.htm\#tab73.

Edwards, J. R., \& Lambert, L. S. (2007). Methods for integrating moderation and mediation: A general analytical framework using moderated path analysis. Psychological Methods, 12(1), 1-22. doi: 10.1037/1082-989X.12.1.1

Emerson, E. (2010). Self-reported exposure to disablism is associated with poorer selfreported health and well-being among adults with intellectual disabilities in England: A cross-sectional survey. Public Health, 124(12), 682-689.

Espelage, D. L., Aragon, S. R., Birkett, M., \& Koenig, B. W. (2008). Homophobic teasing, psychological outcomes, and sexual orientation among high school students: What influence do parents and schools have? School Psychology Review, 37(2), 202-216.

Fange, A., Iwarsson, S., \& Persson, A. (2002). Accessibility to the public environment as perceived by teenagers with functional limitations in a south Swedish town centre. Disabil Rehabil, 24(6), 318-326. doi: 10.1080/09638280110089906

Flynt, S. W., \& Morton, R. C. (2008). Alabama elementary principals perceptions of bullying. Education, 129(2), 187-191.

Friedman, M. S., Koeske, G. F., Silvestre, A. J., Korr, W. S., \& Sites, E. W. (2006). The impact of gender-role nonconforming behavior, bullying, and social support on suicidality among gay male youth. The Journal of Adolescent Health, 38(5), 621623.

Friedman, M. S., Marshal, M. P., Guadamuz, T. E., Wei, C., Wong, C. F., Saewyc, E. (2011). A meta-analysis of disparities in childhood sexual abuse, parental physical abuse, and peer victimization among sexual minority and sexual nonminority individuals. Am J Public Health, 101(8), 1481-1494. doi:

10.2105/ajph.2009.190009 
Fritz, M. S., \& MacKinnon, D. P. (2007). Required sample size to detect the mediated effect. Psychological Science, 18(3), 233-239. doi: 10.1111/j.14679280.2007.01882.x

Garland-Thompson, R. (2004). Integrating disability, transforming feminist theory. In B. G. Smith \& B. Hutchison (Eds.), Gendering disability (pp. 73-103). New Brunswick, NJ: Rutgers University Press.

Geist, R., Grdisa, V., \& Otley, A. (2003). Psychosocial issues in the child with chronic conditions. Best Practice \& Research. Clinical Gastroenterology, 17(2), 141-152.

Gerschick, T. J. (2000). Toward a theory of disability and gender. Signs, 25(4), 12631268. doi: $10.2307 / 3175525$

Gortmaker, S. L., Walker, D. K., Weitzman, M., \& Sobol, A. M. (1990). Chronic conditions, socioeconomic risks, and behavioral problems in children and adolescents. Pediatrics, 85(3), 267-276.

Graham, M., \& Schiele, J. H. (2010). Equality-of-oppressions and anti-discriminatory models in social work: Reflections from the USA and UK. European Journal of Social Work, 13(2), 231-244. doi: 10.1080/13691451003690882

Gruber, J., \& Fineran, S. (2008). Comparing the impact of bullying and sexual harassment victimization on the mental and physical health of adolescents. Sex Roles, 59(1/2), 1-13. doi: 10.1007/s11199-008-9431-5

Haddock, C. K., Rindskopf, D., \& Shadish, W. R. (1998). Using odds ratios as effect sizes for meta-analysis of dichotomous data: A primer on methods and issues. Psychological Methods, 3(3), 339-353. doi: 10.1037/1082-989x.3.3.339

Hammel, J., Jones, R., Smith, J., Sanford, J., Bodine, C., \& Johnson, M. (2008). Environmental barriers and supports to the health, function, and participation of people with developmental and intellectual disabilities: Report from the State of the Science in Aging with Developmental Disabilities Conference. Disabil Health J, 1(3), 143-149. doi: S1936-6574(08)00055-1 [pii] 10.1016/ j.dhjo.2008.05.001

Hancock, A.-M. (2007). When multiplication doesn't equal quick addition: Examining intersectionality as a research paradigm. Perspectives on Politics, 5(1), 63-79. 
Hanish, L. D., \& Guerra, N. G. (2000). The roles of ethnicity and school context in predicting children's victimization by peers. American Journal of Community Psychology, 28(2), 201-223. doi: 10.1023/a:1005187201519

Hatzenbuehler, M. L. (2011). The social environment and suicide attempts in lesbian, gay, and bisexual youth. Pediatrics, 127(5), 896-903. doi: 10.1542/peds.20103020

Hatzenbuehler, M. L., Phelan, J. C., \& Link, B. G. (2013). Stigma as a fundamental cause of population health inequalities. Am J Public Health, 103(5), 813-821. doi: 10.2105/AJPH.2012.301069

Havercamp, S., Roth, M. S., Scandlin, D., Herrick, H., \& Gizlice, Z. (2004). Health and disability in North Carolina 2003: A joint report from the N.C. Office on Disability and Health and the State Center for Health Statistics (pp. 30). Chapel Hill, N.C.: North Carolina Office on Disability and Health.

Hawker, D. S. J., \& Boulton, M. J. (2000). Twenty years' research on peer victimization and psychosocial maladjustment: A meta-analytic review of cross-sectional studies. Journal of Child Psychology \& Psychiatry \& Allied Disciplines, 41(4), 441-455.

Heeringa, S. G., West, B. T., \& Berglund, P. A. (2010a). Applied survey data analysis. Boca Raton, FL: Chapman \& Hall/CRC.

Heeringa, S. G., West, B. T., \& Berglund, P. A. (2010b). Applied survey data analysis (web supplement). Retrieved 4/16/11, 2011, from http://www.isr.umich.edu/src/smp/asda/Additional\%20Stata\%20Examples\%20est at\%20gof.pdf

Herr, N. R. (n.d.). Mediation with dichotomous outcomes. Retrieved September 12, 2011, from http://nrherr.bol.ucla.edu/Mediation/logmed.html

Hershberger, S. L., Pilkington, N. W., \& D'Augelli, A. R. (1997). Predictors of suicide attempts among gay, lesbian, and bisexual youth. Journal of Adolescent Research, 12(4), 477-497. doi: 10.1177/0743554897124004 
Hogan, A., McLellan, L., \& Bauman, A. (2000). Health promotion needs of young people with disabilities: A population study. Disability \& Rehabilitation, 22(8), 352-357. doi: $10.1080 / 096382800296593$

Hollar, D. (2005). Risk behaviors for varying categories of disability in NELS:88. Journal of School Health, 75(9), 350-358. doi: 10.1111/j.17461561.2005.00049.x

Holmbeck, G. N., \& Devine, K. A. (2010). Psychosocial and family functioning in spina bifida. Developmental Disabilities Research Reviews, 16(1), 40-46. doi: 10.1002/ddrr.90

Horwood, J., Waylen, A., Herrick, D., Williams, C., \& Wolke, D. (2005). Common visual defects and peer victimization in children. Investigative Ophthalmology \& Visual Science, 46(4), 1177-1181.

Howe, G. W., Feinstein, C., Reiss, D., Molock, S., \& Berger, K. (1993). Adolescent adjustment to chronic physical disorders--I. Comparing neurological and nonneurological conditions. Journal of Child Psychology \& Psychiatry \& Allied Disciplines, 34(7), 1153-1171.

Hugh-Jones, S., \& Smith, P. K. (1999). Self-reports of short- and long-term effects of bullying on children who stammer. The British Journal of Educational Psychology, 69 ( Pt 2), 141-158.

Hulko, W. (2009). The time-and context-contingent nature of intersectionality and interlocking oppressions. Affilia: Journal of Women \& Social Work, 24(1), 44-55.

Humphrey, J. L., Storch, E., \& Geffken, G. (2007). Peer victimization in children with attention-deficit hyperactivity disorder. Journal of Child Health Care, 11(3), 248260 .

Huurre, T., \& Aro, H. (1998). Psychosocial development among adolescents with visual impairment. European Child \& Adolescent Psychiatry, 7(2), 73-78.

Huurre, T., \& Aro, H. (2000). The psychosocial well-being of Finnish adolescents with visual Impairments versus those with chronic conditions and those with no disabilities. Journal of Visual Impairment \& Blindness, 94(10), 625-638. 
Jones, S. E., \& Lollar, D. J. (2008). Relationship between physical disabilities or longterm health problems and health risk behaviors or conditions among US high school students. Journal of School Health, 78(5), 252-257. doi: 10.1111/j.17461561.2008.00297.x

Judd, C. M., \& Kenny, D. A. (2010). Data analysis in social psychology: Recent and recurring issues. In S. Fiske, D. Gilbert \& G. Lindzey (Eds.), The handbook of social psychology (Vol. 1, pp. 115-139). Boston: McGraw-Hil.

Juvonen, J., \& Galván, A. (2009). Bullying as means to foster compliance. In M. J. Harris (Ed.), Bullying, rejection, and peer victimization: A social cognitive neuroscience perspective (pp. 299-318). NY: Springer Publishing Company.

Juvonen, J., Graham, S., \& Schuster, M. A. (2003). Bullying among young adolescents: The strong, the weak, and the troubled. Pediatrics, 112(6), 1231-1237. doi: 10.1542/peds.112.6.1231

Kenny, D. A. (2008). Mediation with dichotomous outcomes. Retrieved September 12, 2011, from http://davidakenny.net/doc/dichmed.doc

Kenny, D. A. (2011). Mediation. Retrieved 11/19/11, 2011, from http://davidakenny.net/cm/mediate.htm

Kim, Y. S., \& Leventhal, B. (2008). Bullying and suicide: A review. International Journal of Adolescent Medicine And Health, 20(2), 133-154.

Koenes, S. G., \& Karshmer, J. F. (2000). Depression: A comparison study between blind and sighted adolescents. Issues in Mental Health Nursing, 21(3), 269-279.

Korn, E. L., \& Graubard, B. I. (1991). Epidemiologic studies utilizing surveys: Accounting for the sampling design. American Journal of Public Health, 81(9), 1166-1173.

Kosciw, J., Greytak, E., Diaz, E., \& Bartkiewicz, M. J. (2010). The 2009 National School Climate Survey: The experiences of lesbian, gay, bisexual and transgender youth in our Nation's schools. http://www.glsen.org/binarydata/GLSEN_ATTACHMENTS/file/000/001/1675-5.PDF 
Lavigne, J. V., \& Faier-Routman, J. (1992). Psychological adjustment to pediatric physical disorders: A meta-analytic review. Journal of Pediatric Psychology, 17(2), 133-157.

Lazarus, R. S., \& Folkman, S. (1984). Stress, appraisal, and coping. NY: Springer Publishing Company.

Lewis, N. M. (2009). Mental health in sexual minorities: Recent indicators, trends, and their relationships to place in North America and Europe. Health \& Place, 15(4), 1029-1045.

Link, B. G., \& Phelan, J. C. (2001). Conceptualizing stigma. Annual Review of Sociology, 27(1), 363-385.

MacKinnon, D. P. (2008). Introduction to statistical mediation analysis. NY: Lawrence Erlbaum Associates.

MacKinnon, D. P., Fairchild, A. J., \& Fritz, M. S. (2007). Mediation analysis. Annual Review of Psychology, 58(1), 593-614. doi: 10.1146/annurev.psych.58.110405.085542

McCall, L. (2005). The complexity of intersectionality. Signs: Journal of Women in Culture \& Society, 30(3), 1771-1800.

McDonald, K. E., Keys, C. B., \& Balcazar, F. E. (2007). Disability, race/ethnicity and gender: themes of cultural oppression, acts of individual resistance. American Journal of Community Psychology, 39(1/2), 145-161. doi: 10.1007/s10464-0079094-3

Menard, S. (2010). Logistic regression: From introductory to advanced concepts and applications. Thousand Oaks, CA: SAGE Publications, Inc.

Meyer, I. H. (2003). Prejudice, social stress, and mental health in lesbian, gay, and bisexual populations: Conceptual issues and research evidence. Psychological Bulletin, 129(5), 674-697.

Meyer, I. H., Schwartz, S., \& Frost, D. M. (2008). Social patterning of stress and coping: Does disadvantaged social statuses confer more stress and fewer coping 
resources? Social Science \& Medicine, 67(3), 368-379. doi: DOI: 10.1016/j.socscimed.2008.03.012

Miauton, L., Narring, F., \& Michaud, P.-A. (2003). Chronic illness, life style and emotional health in adolescence: Results of a cross-sectional survey on the health of 15-20-year-olds in Switzerland. European Journal of Pediatrics, 162(10), 682689.

Mitchell, D. D. (2006). Flashcard: Alternating between visible and invisible identities. Equity \& Excellence in Education, 39(2), 137-145.

Montana Office of Public Instruction. (2011). 2011 Montana Youth Risk Behavior Survey: High school results: Students with Disabilities. Retrieved from http://www.opi.mt.gov/pdf/YRBS/11/Reports/11StudentsWithDisabilities.pdf.

Moy, L. C. (2008). Disrupting "bully talk": Progressive practices and transformative spaces for anti-violence work in schools. (PhD Doctoral), University of British Columbia, Vancouver. Retrieved from http://hdl.handle.net/2429/5739

Nabuzoka, D., Rønning, J. A., \& Handegård, B. H. (2009). Exposure to bullying, reactions and psychological adjustment of secondary school students. Educational Psychology, 29(7), 849-866.

Nash, J. C. (2008). Re-thinking intersectionality. Feminist Review, 89(1), 1-15. doi: $10.1057 /$ fr. 2008.4

O'Toole, C. J. (2000). The view from below: Developing a knowledge base about an unknown population. Sexuality \& Disability, 18(3), 207-224.

Olweus, D. (1993). Bullying at school: What we know and what we can do. Cambridge, MA: Blackwell.

Oregon Department of Human Services. (2008). Oregon Healthy Teens Survey: 2008 11th grade. Portland: Oregon Health Authority Retrieved from http://www.dhs.state.or.us/dhs/ph/chs/youthsurvey/ohteens/2008/oht11th2008.pdf 
Orpinas, P., \& Horne, A. M. (2005). Bullying prevention: Creating a positive school climate and developing social competence. Washington D.C.: American Psychological Association.

Pascoe, C. J. (2011). Dude, you're a fag. Berkeley: University of California Press.

Peng, C.-Y. J., \& So, T.-S. H. (2002). Logistic regression analysis and reporting: A primer. Understanding Statistics, 1(1), 31-70. doi: 10.1207/S15328031US0101_04

Petersen, A. J. (2006). An African American woman with disabilities: The intersection of gender, race and disability. Disability \& Society, 21(7), 721-734.

Petersen, A. J., \& Gallagher, D. J. (2006). Exploring intersectionality in education: The intersection of gender, race, disability, and class. (PhD Doctoral), University of Northern Iowa.

Phelan, J. C., Link, B. G., \& Dovidio, J. F. (2008). Stigma and prejudice: One animal or two? Social Science \& Medicine, 67(3), 358-367.

Preacher, K. J., Rucker, D. D., \& Hayes, A. F. (2007). Addressing moderated mediation hypotheses: Theory, methods, and prescriptions. Multivariate Behavioral Research, 42(1), 185-227. doi: 10.1080/00273170701341316

Purdie-Vaughns, V., \& Eibach, R. (2008). Intersectional invisibility: The distinctive advantages and disadvantages of multiple subordinate-group identities. Sex Roles, 59(5/6), 377-391. doi: 10.1007/s11199-008-9424-4

Reiter, S., Bryen, D. N., \& Shachar, I. (2007). Adolescents with intellectual disabilities as victims of abuse. Journal of Intellectual Disabilities, 11(4), 371-387.

Rumpf, H.-J., Meyer, C., Hapke, U., \& John, U. (2001). Screening for mental health: validity of the MHI-5 using DSM-IV Axis I psychiatric disorders as gold standard. Psychiatry Research, 105(3), 243-253. doi: Doi: 10.1016/s01651781(01)00329-8 
Safren, S. A., \& Heimberg, R. G. (1999). Depression, hopelessness, suicidality, and related factors in sexual minority and heterosexual adolescents. Journal of Consulting \& Clinical Psychology, 67(6), 859-866.

Sawyer, A. L., Bradshaw, C. P., \& O'Brennan, L. M. (2008). Examining ethnic, gender, and developmental differences in the way children report being a victim of "bullying" on self-report measures. The Journal of Adolescent Health, 43(2), 106114.

Sen, G., Iyer, A., \& Mukherjee, C. (2009). A methodology to analyse the intersections of social inequalities in health. Journal of Human Development \& Capabilities, 10(3), 397-415. doi: 10.1080/19452820903048894

Sentenac, M., Gavin, A., Arnaud, C., Molcho, M., Godeau, E., \& Gabhainn, S. N. (2011). Victims of bullying among students with a disability or chronic illness and their peers: A cross-national study between Ireland and France. The Journal of Adolescent Health, 48(5), 461-466.

Shuttleworth, R. P. (2004). Disabled masculinity: Expanding the masculine repertoire. In B. G. Smith \& B. Hutchison (Eds.), Gendering disability (pp. 166-178). New Brunswick, NJ: Rutgers University Press.

Simeonsson, R. J., Carlson, D., Huntington, G. S., McMillen, J. S., \& Brent, J. L. (2001). Students with disabilities: A national survey of participation in school activities. Disability \& Rehabilitation, 23(2), 49-63. doi: 10.1080/096382801750058134

Spriggs, A. L., Iannotti, R. J., Nansel, T. R., \& Haynie, D. L. (2007). Adolescent bullying involvement and perceived family, peer and school relations: commonalities and differences across race/ethnicity. The Journal of Adolescent Health, 41(3), 283293.

StataCorp. (2007). Stata survey data reference manual: Release 10. College Station, TX: StataCorp LP.

StataCorp. (2011). Stata survey data reference manual: Release 12. College Station, TX: StataCorp LP. 
Stein, R. E., \& Jessop, D. J. (1982). A noncategorical approach to chronic childhood illness. Public Health Reports (Washington, D.C.: 1974), 97(4), 354-362.

Stevens, S. E., Steele, C. A., Jutai, J. W., Kalnins, I. V., Bortolussi, J. A., \& Biggar, W. D. (1996). Adolescents with physical disabilities: Some psychosocial aspects of health. The Journal of Adolescent Health, 19(2), 157-164.

Strand, B. H., Dalgard, O. S., Tambs, K., \& Rognerud, M. (2003). Measuring the mental health status of the Norwegian population: A comparison of the instruments SCL25, SCL-10, SCL-5 and MHI-5 (SF-36). Nordic Journal of Psychiatry, 57(2), 113-118.

Stuart, O. W. (1992). Race and disability: Just a double oppression? Disability \& Society, $7(2), 177-188$.

Surís, J. C., Parera, N., \& Puig, C. (1996). Chronic illness and emotional distress in adolescence. The Journal of Adolescent Health, 19(2), 153-156.

Surís, J. C., Resnick, M. D., Cassuto, N., \& Blum, R. W. (1996). Sexual behavior of adolescents with chronic disease and disability. The Journal of Adolescent Health, 19(2), 124-131.

Sweeting, H., \& West, P. (2001). Being different: Correlates of the experience of teasing and bullying at age 11. Research Papers in Education, 16(3), 225-246.

Tabachnick, B. G., \& Fidell, L. S. (2007). Using multivariate statistics (5th ed.). Boston: Pearson Education, Inc.

Tate, D., Forchheimer, M., Maynard, F., \& Dijkers, M. (1994). Predicting depression and psychological distress in persons with spinal cord injury based on indicators of handicap. American Journal of Physical Medicine \& Rehabilitation, 73(3), 175183.

Thomas, S., \& Heck, R. (2001). Analysis of large-scale secondary data in higher education research: Potential perils associated with complex sampling designs. Research in Higher Education, 42(5), 517-540. doi: 10.1023/a:1011098109834 
Thompson, R. J., \& Gustafson, K. E. (1996). Adaptation to chronic childhood illness. Washington, DC: American Psychological Association.

Toomey, R. B., Ryan, C., Diaz, R. M., Card, N. A., \& Russell, S. T. (2010). Gendernonconforming lesbian, gay, bisexual, and transgender youth: School victimization and young adult psychosocial adjustment. Developmental Psychology, 46(6), 1580-1589.

UCLA: Statistical Consulting Group. (2012). Chapter 3 Logistic Regression Diagnostics. Retrieved 6/16/12, 2012, from http://www.isr.umich.edu/src/smp/asda/Additional\%20Stata\%20Examples\%20sg mediation\%20with\%20svy\%20and\%20subpopulation.pdf

Udry, J. R. (1998). The National Longitudinal Study of Adolescent Health (Add Health), Waves I \& II, 1994-1996 [machine-readable data file and documentation]. Chapel Hill, NC: Carolina Population Center, University of North Carolina at Chapel Hill.

Unnever, J. D., \& Cornell, D. G. (2003). Bullying, self-control, and adhd. Journal of Interpersonal Violence, 18(2), 129-147.

van Cleave, J., \& Davis, M. M. (2006). Bullying and peer victimization among children with special health care needs. Pediatrics, 118(4), e1212-e1219.

van der Lee, J. H., Mokkink, L. B., Grootenhuis, M. A., Heymans, H. S., \& Offringa, M. (2007). Definitions and measurement of chronic health conditions in childhood: A systematic review. JAMA, 297(24), 2741-2751. doi: 10.1001/jama.297.24.2741

Varni, J. W., \& Setoguchi, Y. (1992). Screening for behavioral and emotional problems in children and adolescents with congenital or acquired limb deficiencies. American Journal of Diseases of Children, 146(1), 103-107.

Varni, J. W., Setoguchi, Y., Rappaport, L. R., \& Talbot, D. (1991). Effects of stress, social support, and self-esteem on depression in children with limb deficiencies. Archives of Physical Medicine \& Rehabilitation, 72(13), 1053-1058. 
Veit, C. T., \& Ware, J. E. (2008). Part 2: Measures: Mental Health Inventory (MHI). In A. J. Rush, M. B. First \& D. Blacker (Eds.), Handbook of psychiatric measures (2nd ed., pp. 65-67). Washington, D.C.: Amerian Psychiatric Publishing, Inc.

Wagner, M., Newman, L., Cameto, R., Levine, P., \& Marder, C. (2007). Perceptions and expectations of youth with disabilities. A special topic report from the National Longitudinal Transition Study-2 (NLTS2) (NCSER 2007-3006). http://www.nlts2.org

Waldo, C. R., Hesson-McInnis, M. S., \& D'Augelli, A. R. (1998). Antecedents and consequences of victimization of lesbian, gay, and bisexual young people: A structural model comparing rural university and urban samples. American Journal of Community Psychology, 26(2), 307-334. doi: 10.1023/a:1022184704174

Wallander, J. L., Thompson, R., \& Alriksson-Schmidt, A. I. (2003). Pscyhological adjustment of children with chronic physical conditions. In M. C. Roberts (Ed.), Handbook of pediatric psychology (3rd ed., pp. 141-158). NY: Guilford Press.

Wallander, J. L., \& Varni, J. W. (1995). Appraisal, coping, and adjustment in adolescents with a physical disability. In J. L. Wallander \& L. J. Siegel (Eds.), Adolescent health problems: Behavioral perspectives (pp. 209-231). New York: Guilford Press.

Wang, J., Iannotti, R. J., Luk, J. W., \& Nansel, T. R. (2010). Co-occurrence of victimization from five subtypes of bullying: Physical, verbal, social exclusion, spreading rumors, and cyber. Journal of Pediatric Psychology, 35(10), 11031112 .

Wang, J., Iannotti, R. J., \& Nansel, T. R. (2009). School bullying among adolescents in the United States: Physical, verbal, relational, and cyber. The Journal of Adolescent Health, 45(4), 368-375.

Ware, J. E., Kosinski, M., Bjorner, J. B., Turner-Bowker, D. M., Gandek, B., \& Maruish, M. E. (2007). User's manual for the $S F-36 v 2^{T M}$ health survey (Q. Incorporated Ed. 2nd ed.). Lincoln, RI: QualityMetric Incorporated.

WestEd, \& California Dept. of Education. (2008). Query CHKS (Online database). Retrieved 4/2/11, from WestEd http://chks.wested.org/indicators 
Wiener, J., \& Mak, M. (2009). Peer victimization in children with attentiondeficit/hyperactivity disorder. Psychology in the Schools, 46(2), 116-131.

Witt, W. P., Riley, A. W., \& Coiro, M. J. (2003). Childhood functional status, family stressors, and psychosocial adjustment among school-aged children with disabilities in the United States. Arch Pediatr Adolesc Med, 157(7), 687-695. doi: 10.1001/archpedi.157.7.687

Wolman, C., Resnick, M. D., Harris, L. J., \& Blum, R. W. (1994). Emotional well-being among adolescents with and without chronic conditions. The Journal of Adolescent Health, 15(3), 199-204.

World Health Organization. (2001). International classification of functioning, disability and health. Geneva: World Health Organization.

Wright, R., E. (1994). Logistic regression. In L. Grimm, G. \& P. Yarnold, R. (Eds.), Reading and understanding multivariate statistics (pp. 217-244). Washington D.C.: American Psychological Association.

Yamazaki, S., Fukuhara, S., \& Green, J. (2005). Usefulness of five-item and three-item Mental Health Inventories to screen for depressive symptoms in the general population of Japan. Health \& Quality of Life Outcomes, 3(1), 1-7. doi: $10.1186 / 1477-7525-3-48$

Young, I. M. (1990). Justice and the politics of difference. New Jersey: Princeton University Press.

Young, R., \& Sweeting, H. (2004). Adolescent bullying, relationships, psychological well-being, and gender-atypical behavior: A gender diagnosticity approach. Sex Roles, 50(7/8), 525-537. 


\section{APPENDIX A: Human Subjects Approval from the PSU HSRRC}

$\begin{array}{ll}\text { Post Office Box } 751 & 503-725-4288 \text { tel } \\ \text { Portland, Oregon 97207-0751 } & 503-725-8170 \text { fax } \\ & \text { hsrrcelists.pdx.edu }\end{array}$

To: $\quad$ Marjorie G. McGee

From: HSSRC 2012

Date: January 13,2012

Re: HSRRC waived review of your application titled, "Lost in the Margins: Intersections among Disability and Other Non-Dominant Statuses with Regards to Peer Victimization and Psychosocial Distress among Oregon Teens" (HSRRC \# 122009)

Your proposal is exempt from further Human Subjects Research Review Committee review, and you may proceed with the study.

Even with the exemption above, it was necessary by University policy for you to notify this Committee of the proposed research, and we appreciate your timely attention to this matter. If you make changes in the research protocol, the Committee must be notified in writing, and changes must be approved before being implemented.

If you have questions or concerns, please contact the HSRRC in the Office of Research and Strategic Partnerships (RSP), (503) 725-2243, Market Center Building, $6^{\text {th }}$ floor.

cc: Laurie Powers 


\section{APPENDIX B: 2010 Data agreement: Oregon Health Authority}

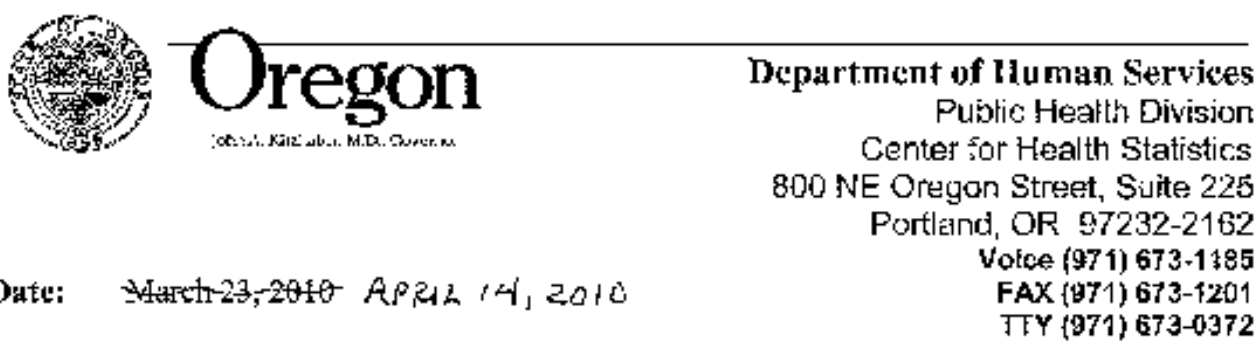

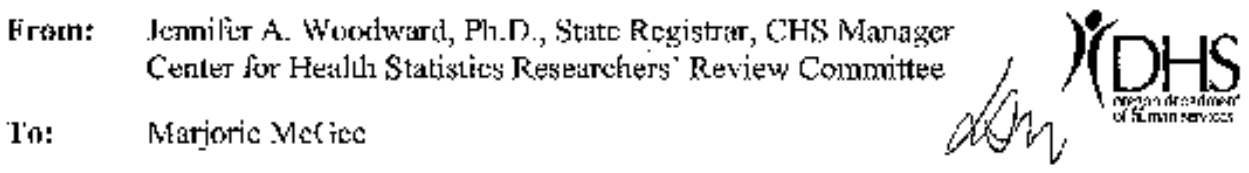

Organizatiun: PHDiOFH/Adolc5ccnt Henlth

Subject: Notice of Review of Your Data-use Request, dated 2-18-10

Study Iitle: A.MCHP Presonesption I. Laming Collabs'ative

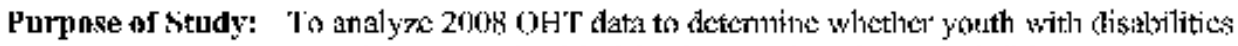
experience more health risks that youth without disabilities.

\section{Committee Response to your request:}

Di Graned

2008 (Jegen Healthy Tecons Survey datat

Need AdditionaI information:

Granted with Conditions (see explanation below):

I I Darical (sect explatral.ron beluw)

Important Nate: Ilense notify me of awy project moditication that would change how the requested data are uscd, or now projects where these diats will he used. 
APPENDIX C: 2012 Data agreement: Oregon Health Authority

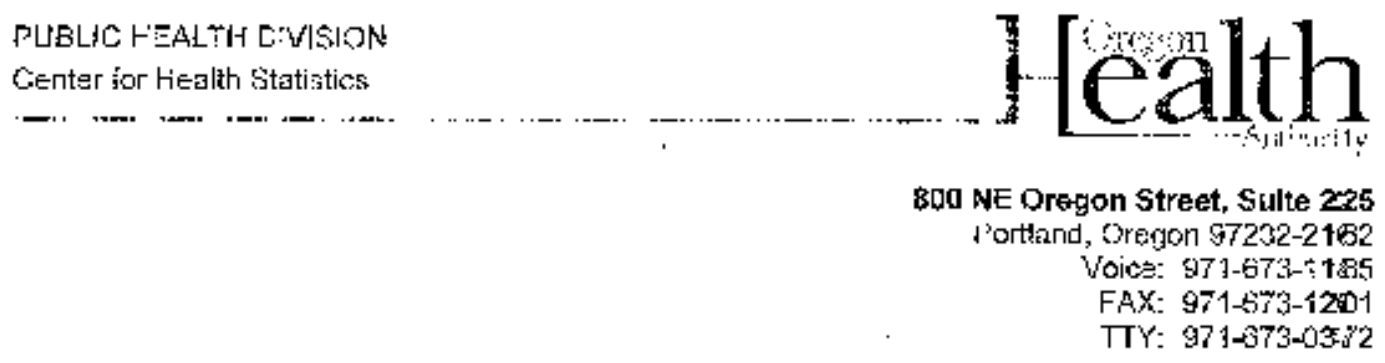

Date: +hneary 9,2012

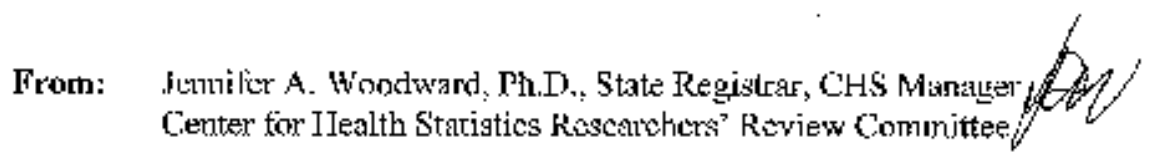

To: Marjoric McGe

Organization: Poriland State duniversity

Sulject: Approval of use or 2008 Orewron Ilealthy Teens Data

\section{Study Title: Dissertation Rescarelı}

Purpuse of Study: The purpose of the siudy is to defermine whether your sith jisubilities expericuce more health risk than youtlı witbout disabilitiey.

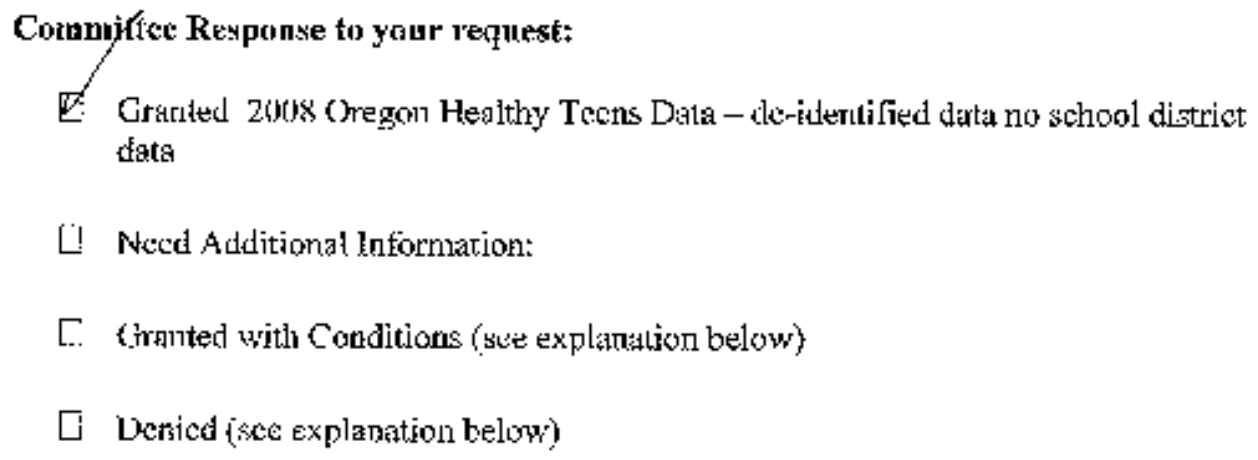

L! Necd Additionat lnformation:

[. Cirnnted with Conditions (sue explanation below)

Dersicd (sce explanation below)

Important Yote: Plensc notily ne of any project modification that would ehange bow the requested datil are used, or now projects where these data will be used. 